\title{
Palladium-Catalyzed Carboxy-Alkynylation of Propargylic Amines Using Carbonate Salts as Carbon Dioxide Source
}

\author{
Phillip D. G. Greenwood and Jerome Waser ${ }^{\star[a]}$
}

\begin{abstract}
A palladium-catalyzed multi-component reaction of propargylic amines, alkynyl bromides and cesium hydrogen carbonate to access oxazolidinones is reported. In contrast to previous reports, only a slight excess of cesium hydrogen carbonate is used as surrogate of carbon dioxide. The reaction gives access to oxazolidinones bearing alkyl- and aryl polysubstituted enynes in good yield and very high $E$ stereoselectivity.
\end{abstract}

\section{Introduction}

The utilisation of the greenhouse gas $\mathrm{CO}_{2}$ has attracted significant interest over recent years. ${ }^{[1]}$ For the organic chemistry community, focus has been on incorporating $\mathrm{CO}_{2}$ into organic molecules for the synthesis of valuable compounds and commodity chemicals. ${ }^{\left[{ }^{[2]}\right.}$ As an important application, insertion of $\mathrm{CO}_{2}$ into propargylic amines to form oxazolidinones has been achieved using a range of diverse conditions (Scheme 1). . $^{3,4]}$ Transformations without metal catalysts require harsh or specialized conditions, such as super base ${ }^{[3 a-d]}$ or super critical $\mathrm{CO}_{2}{ }^{[3, f]} \mathrm{A}$ number of transition-metal catalysed processes allowing milder $r$ eaction conditions have been developed using ruthenium ${ }^{[4 a]}$ palladium, ${ }^{[4 b, c]}$ silver ${ }^{[4 d-i]}$ gold ${ }^{[4 j, k]}$ and zinc ${ }^{[4]]}$ catalysts, as well as a copper substituted ionic liquid. ${ }^{[4 \mathrm{~m}]} \mathrm{In}$ all of these reported methods, the addition of $\mathrm{CO}_{2}$ has been combined with protonation of the triple bond in the propagylic amines, resulting in the formation of a $\mathrm{C}-\mathrm{O}$ and a $\mathrm{C}-\mathrm{H}$ bond (Scheme $1 \mathrm{~A}){ }^{[5]}$ Recently the Nevado group demonstrated through the use of palladium catalysis that a carboxylation-arylation sequence can be performed as part a new multicomponent synthesis of oxazolidinones (Scheme 1B)..$^{[6]}$ Using gaseous $\mathrm{CO}_{2}$ at atmospheric pressure, they obtained tetra-substituted alkenes via $\mathrm{C}-\mathrm{O}$ and $\mathrm{C}-\mathrm{C}$ bond formation, allowing for a broader range of oxazolidinones to be synthesised. Developing efficient methods to access oxazolidinones is important, as they are useful as intermediates ${ }^{[7]}$ or chiral auxiliaries ${ }^{[8]}$ in organic synthesis. They are also found in agrochemicals and antibacterial drugs ${ }^{[9]}$ and their material properties have even been exploited for applications in lithium battery technology. ${ }^{[10]}$

As most carboxylation reactions, the developed approaches are usually based on the use of gaseous $\mathrm{CO}_{2}$, which is convenient as it is widely available. However, the use of a gas leads to inefficient mass transport at the gas/liquid interface, ${ }^{[11]}$ resulting in a poor atom economy. For example, a $1 \mathrm{mmol}$ reaction with a 2 litre balloon of $\mathrm{CO}_{2}$ has an excess of 89 equivalents of $\mathrm{CO}_{2}$. Therefore, it is sometimes advantageous to have a solid $\mathrm{CO}_{2}$ source to enable easier handling and better reaction efficiency. In this context, a limited number of examples have been reported with a carbonate salt as source of $\mathrm{CO}_{2}$ in a reaction or acting directly as a nucleophile/electrophile. ${ }^{[3, b, b, 12]}$ In 1996, Costa and co-workers developed an organic super base catalysed carboxylation of propargylic amines. This publication contained a single example of the use of a tetramethylguanidinium carbamate amine complex as a carrier for $\mathrm{CO}_{2}{ }^{[3 a]}$ In a subsequent work two years later they showed the use of sodium hydrogen carbonate as the $\mathrm{CO}_{2}$ source. ${ }^{[3 \mathrm{~b}]}$ The reaction between epoxide and sodium hydrogen carbonate catalysed by tetrabutylammonium bromide was also developed as a viable pathway to access cyclic carbonates. ${ }^{[12]}$ Trost and co-workers reported the use of carbonates in the palladium-catalyzed enantioselective ringopening of vinyl epoxides to give either diols or cyclic carbonates. ${ }^{[13]}$

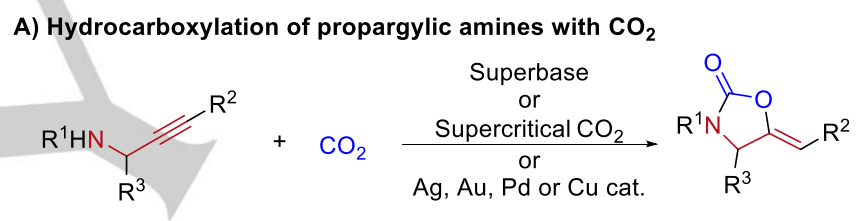

B) Nevado: Aryl-carboxylation of propargylic amines with $\mathrm{CO}_{2}$

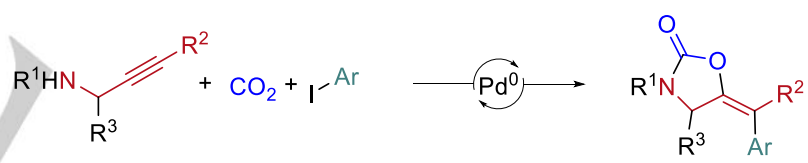

C) This work: Alkynyl-carboxylation of propargylic amines with $\mathrm{CsHCO}_{3}$

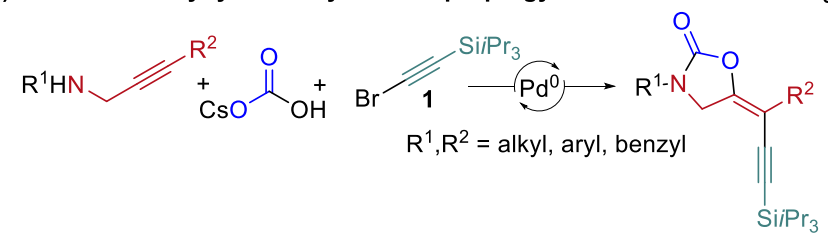

Scheme 1. Synthesis of oxazolidinones from propargylic amines.

Herein, we describe a convenient method for the palladium catalysed three-component synthesis of oxazolidinones from propargylic amines with cesium hydrogen carbonate as the $\mathrm{C} 1$ source instead of gaseous $\mathrm{CO}_{2}$ (Scheme 1C). Alkynyl bromide 1 was used as a third partner, resulting in a new stereoselective synthesis of highly substituted enynes. ${ }^{[14]}$

\section{Results and Discussion}

While working on the palladium catalysed oxyalkynylation of propargylic amines tethered by trifluoroacetaldehyde 
derivatives, ${ }^{[15]}$ an unexpected side reaction was observed: When the reaction of propargylic amine $2 \mathrm{a}$ with silyl alkynyl bromide 1 and half acetal $\mathbf{3}$ was examined, oxazolidinone product $\mathbf{5 a}$ was observed in addition to the expected product 4 (Scheme 2). This was confusing, as there was no obvious source of $\mathrm{CO}_{2}$ in the reaction. When the reaction was performed in the absence of hemiacetal tether with or without a balloon of $\mathrm{CO}_{2}$, exclusive formation of oxazolidinone $\mathbf{5} \mathbf{a}$ was observed. The formation of $\mathbf{5 a}$ could be finally suppressed to give 4 in high yield by the use of non-carbonate bases, establishing the latter as the source of the $\mathrm{CO}_{2}$ group. When considering the lack of reports in the use of carbonate salts in the palladium-catalyzed multi-component synthesis of oxazolidinones, combined with the fact that enynes were never reported as products, we decided to optimize the formation of 5 a. $\mathrm{CsHCO}_{3}$ was finally identified as the best $\mathrm{CO}_{2}$ source, with a palladium-DPEPhos complex as catalyst in DCE at $60{ }^{\circ} \mathrm{C}$ (Table 1, entry 1). Under these conditions, product 5a was obtained in $77 \%$ isolated yield.
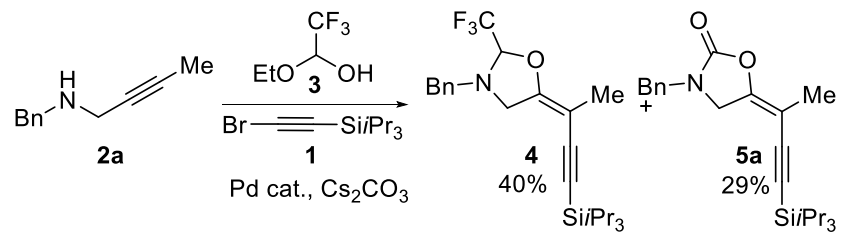

Scheme 2. Unexpected formation of oxazolidinone $\mathbf{5 a}$ observed during the tethered oxyalkynylation of propargylic amine $\mathbf{2 a}$ impact on the outcome of the reaction. The carbonate salts of both cesium and rubidium gave the desired product albeit with modest yield (entry 2). The use of salts of smaller cations gave poor yields and calcium and barium carbonates gave no product formation at all (entry 3). A higher temperature of $75^{\circ} \mathrm{C}$ (entry 4), and a lower temperature of $50{ }^{\circ} \mathrm{C}$ (entry 5 ) both led to a lower yield of $64 \%$, with the latter giving incomplete conversion. The choice of ligand played a central role in the oxyalkynylation reaction using hemiacetal tether $3{ }^{[15,16]}$ Therefore, we examined again the most successful ligands from the previous protocol (DCEPhos, XantPhos and dppf). However, they led to the the formation of oxazolidinone 5a in low yields only (entries 6-8). Different solvents (entries 9-11) and palladium sources were also explored (see Supporting Information), but none of the changes provided any improvement. Both the palladium loading (entry 12) and the equivalence of base (entry 13) could be decreased. However, this led to longer reaction time and incomplete conversion. Finally, when the reaction was carried out with a balloon of $\mathrm{CO}_{2}$, the same yield was obtained (entry 14). The addition of $4 \AA$ molecular sieves had no influence on the reaction outcome (entry 15). The transformation was clean and only starting material and product were observed.

With the optimised conditions in hand, we moved on to study the scope of the reaction (Scheme 3). We start by looking at aliphatic groups on the nitrogen; para-methoxy benzyl and ferrocenylmethyl protected propagylic amines were used succesfully in the reaction to give $\mathbf{5 b}$ and $\mathbf{5 c}$. On the alkyne, a primary benzyl (5d) and a secondary cyclopropyl (5e) groups were both well tolerated in the reaction. The tert-butyl substituted enyne $\mathbf{5 f}$ was obtained in lower yields and required longer reaction times and higher temperature for full conversion. We then moved on to propargylic amines bearing aromatic groups on the alkyne. N-benzyl-3-phenylprop-2-yn-1-amine with an unsubstituted phenyl ring gave enyne $\mathbf{5 g}$ in $81 \%$ yield. Substituting the nitrogen with a methyl group gave $5 \mathrm{~h}$ with a reduced yield of $66 \%$. 4-Methoxy (5i), 4-trifluromethyl $(5 \mathbf{j})$ and 3bromo (5k) substituents were tolerated on the aryl ring, giving moderate to good yields. Introducing two methyl substituents at the meta positions (5I) had no detrimental effect on the reaction. Finaly, a propargylic amine with a heteroaromatic thiophene group on the alkyne gave enyne $5 \mathrm{~m}$ in $47 \%$ yield. Substrates with aromatic substituents on the alkyne required increased reaction temperatures and longer reaction time to reach full conversion. All reactions gave a single isomer of the product and the $E$ configuration of the olefin was established by $X$-ray analysis on compound $5 \mathbf{g} .{ }^{[17]}$ The configuration of the other compounds was assumed to be the same based on the similar NMR spectra.

Table 1. Optimisation of the carboxylation-alkynylation of $2 \mathbf{a}^{\left[{ }^{[a]}\right.}$

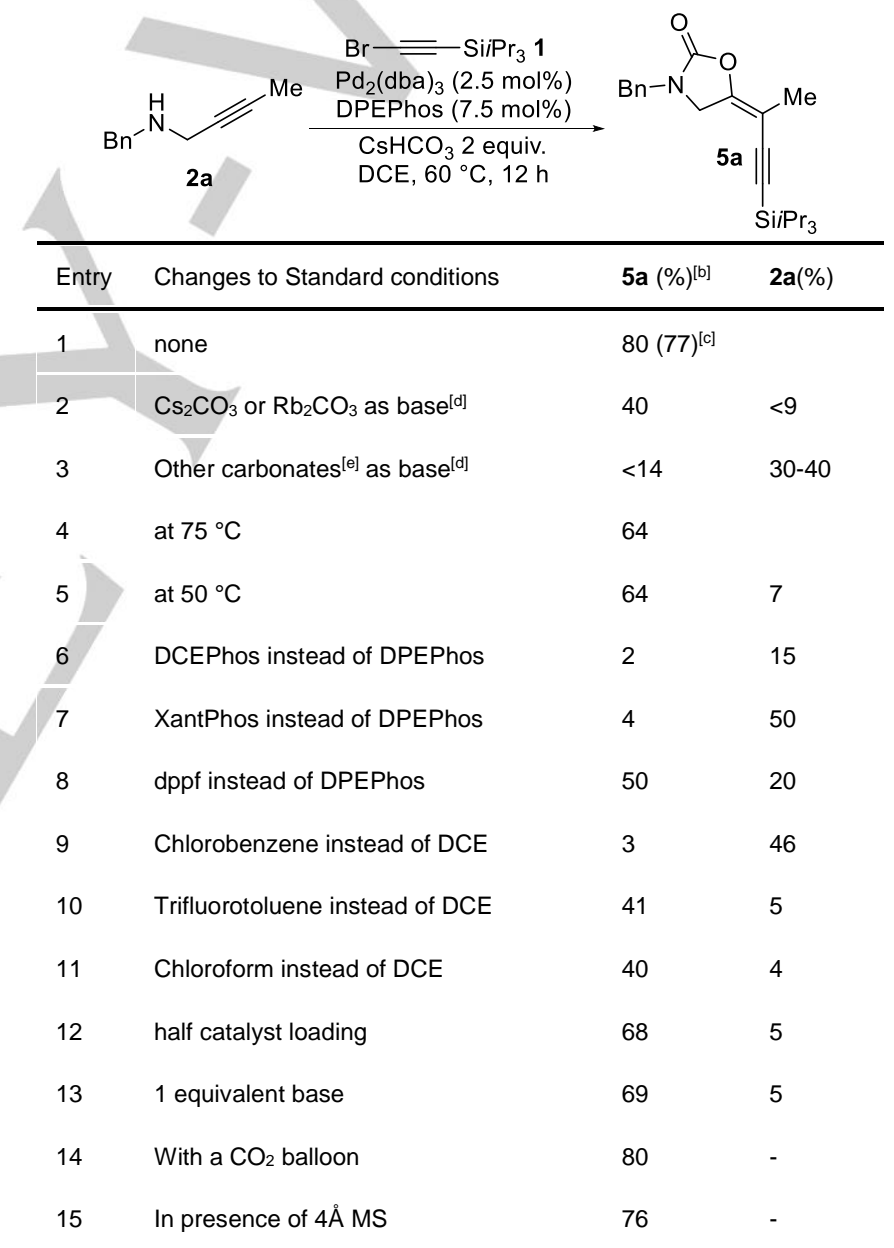

[a] Reaction conditions: $0.10 \mathrm{mmol} 2 \mathrm{a}, 0.13 \mathrm{mmol} \mathbf{1}, 0.20 \mathrm{mmol} \mathrm{CsHCO}_{3}$ in DCE at $60^{\circ} \mathrm{C}$. [b] NMR yields using $p$-difluorobenzene as internal standard. [c] Isolated yield. [d] Reactions run at $75{ }^{\circ} \mathrm{C}$ [e] $\mathrm{Li}_{2} \mathrm{CO}_{3}, \mathrm{Na}_{2} \mathrm{CO}_{3}, \mathrm{~K}_{2} \mathrm{CO}_{3}$, $\mathrm{NaHCO}_{3}, \mathrm{CaCO}_{3}$, and $\mathrm{BaCO}_{3}$ were examined.

When a terminal acetylene substrate, N-benzyl propynylamine, was subjected to the same reaction conditions, only trace amounts of carboxylation product was observed. The use of substrates with substituents at the propargylic position or an attempt of carboxy-arylation as well as carboxy-alkynylation 
with aryl- or alkyl- substituted bromoalkynes were also not successful, even with the ligands applied to these classes of substrates in the aminal-tethered oxyalkynylation reaction (trifuryl phosphine and RuPhos). These reactions were also performed under $\mathrm{CO}_{2}$ atmosphere, but the carboxylation products were still not observed.

The mechanism that is generally proposed for the carboxylation of propargylic amines involves the nucleophilic attack of the nitrogen onto the carbon of $\mathrm{CO}_{2}$ to give a carbamate anion which then undergoes a 5-exo-dig cyclisation onto the triple bond activated by palladium (Scheme 4A). In our case, this seems unlikely, as the carbon of the hydrogen carbonate anion is much less electrophilic and the reaction also works with carbonates. It may be possible that the carbonate is undergoing decomposition under the reaction conditions to produce $\mathrm{CO}_{2}$. However, this seems unlikely as the yields obtained would be very high for the generation of only one or two equivalents of $\mathrm{CO}_{2}$ gas. Furthermore, many palladium-catalyzed reactions use carbonate bases under similar conditions, but report of $\mathrm{CO}_{2}$ incorporation remains exceedingly rare, speaking against an efficient generation of gaseous $\mathrm{CO}_{2}$ from the carbonates.

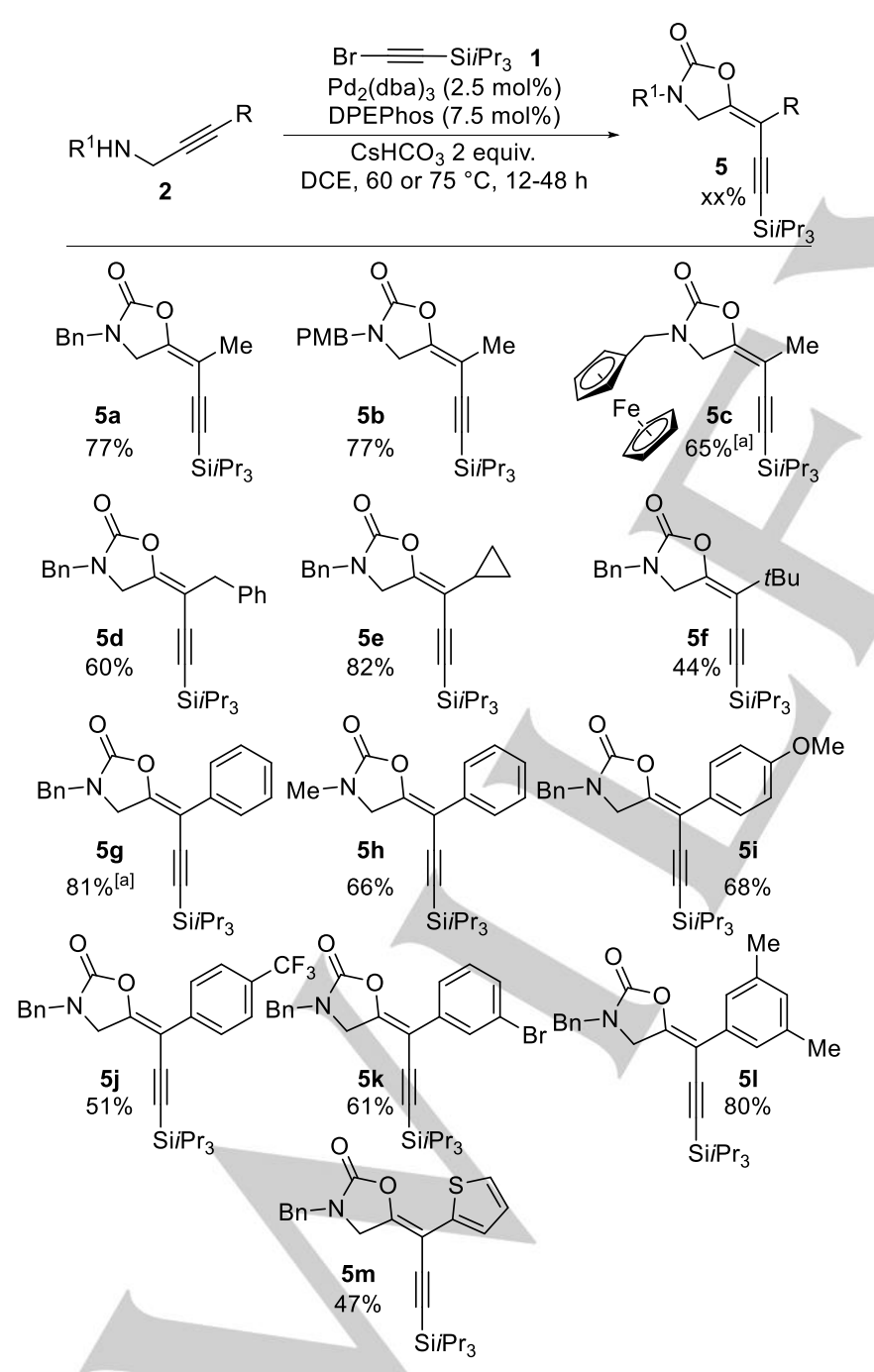

Scheme 3. Scope of the carboxylation-alkynylation reaction. [a] Product isolated with $1-7.5 \%$ dba as impurity. Substrates with aromatic alkynyl groups required $24-48 \mathrm{~h}$ at $75^{\circ} \mathrm{C}$
We propose therefore an alternative pathway for the mechanism of this reaction (Scheme 4B). First, oxidative addition of the palladium(0) complex on alkynyl bromide $\mathbf{1}$ generates palladium(II) complex I. Coordination of the alkyne substrate leads then to activation of the triple bond (II). ${ }^{[18]}$ This step most probably requires first dissociation of the bromide anion to generate a more reactive cationic palladium intermediate with a free coordination site. ${ }^{[19]}$ The carbonate attacks as a nucleophile, ${ }^{[13,20]}$ to give trans oxy-palladation intermediate III. Intramolecular nucleophilic attack by the nitrogen gives with elimination of water gives then vinyl palladium complex IV. Reductive elimination finally leads to the observed product. In this scenario, the oxypalladation step would probably not occur with high regioselectivity, but may be reversible. Fast formation of the oxazolidinone product is possible only for the drawn regioisomer. Alternatively, hydrogen bonding between the amine and the carbonate may direct the attack of the nucleophile. Further investigation will be needed to support this mechanism hypothesis.

A) Established mechanism for carboxylation with $\mathrm{CO}_{2}$<smiles>[R]CNCC(=O)C(C)=O</smiles>

B) Proposed mechanism for carboxylation with carbonate

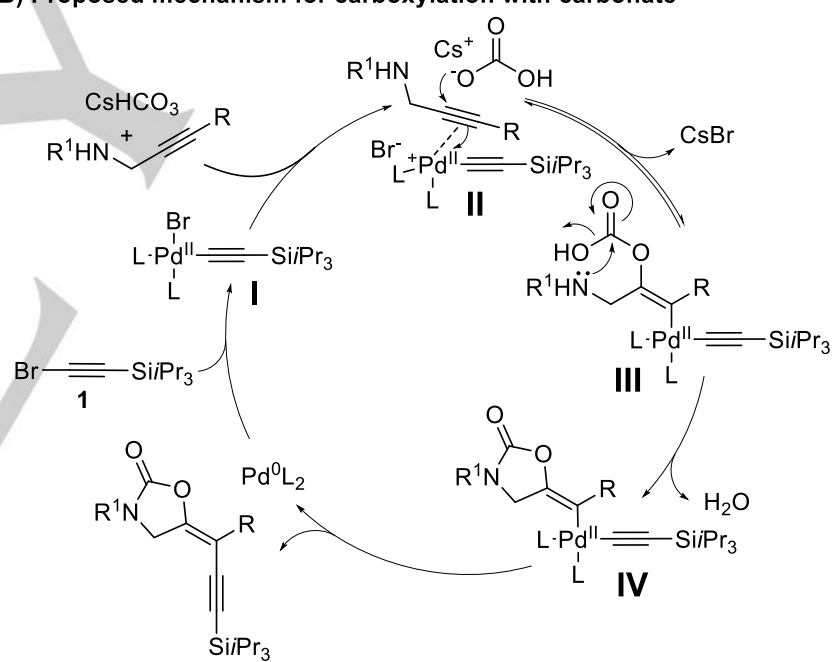

Scheme 4. Proposed mechanism for the carboxy-alkynylation reaction.

\section{Conclusions}

In summary, we have presented an unprecedented palladium catalyzed carboxylation-alkynylation reaction using a solid inorganic hydrogen carbonate source of $\mathrm{CO}_{2}$. It gives access to highly substituted enynes substrates with very high stereoselectivity. The exceptional efficiency of the reaction may suggest an interesting mechanism pathway, which should be more strongly supported by further studies.

\section{Acknowledgments}

This work is supported by the Swiss National Science Foundation (No. 200021_159920), the European Research Council (ERC 
Consolidator Grant No. 771170 and EPFL. We thank Dr. R. Scopelliti and Dr. F. F. Tirani from ISIC at EPFL for X-ray analysis.

Keywords: palladium catalysis $\cdot$ alkynes $\cdot$ heterocycles $\bullet$ carbon dioxide $\cdot$ multi-component reactions

[1] a) D. L. Tomasko, H. Li, D. Liu, X. Han, M. J. Wingert, L. J. Lee, K. W. Koelling, Ind. Eng. Chem. Res. 2003, 42, 6431; K. Sumida, D. L. Rogow, J. A. Mason, T. M. McDonald, E. D. Bloch, Z. R. Herm, T. H. Bae, J. R. Long, Chem. Rev. 2012, 112, 724-781. b) M. Poliakoff, W. Leitner, E. S. Streng, Faraday Discuss. 2015, 183, 9; c) E. Alper, O. Y. Orhan, Petroleum 2017, 3, 109; d) E. I. Koytsoumpa, C. Bergins, E. Kakaras, J. Supercrit. Fluids 2018, 132, 3.

[2] a) T. Sakakura, J.-C. Choi, H. Yasuda, Chem. Rev. 2007, 107, 2365; b) M. Cokoja, C. Bruckmeier, B. Rieger, W. A. Herrmann, F. E. Kuhn, Angew. Chem., Int. Ed. 2011, 50, 8510; c) Q. Liu, L. P. Wu, R. Jackstell M. Beller, Nature Commun. 2015, 6; d) A. Tortajada, F. Julia-Hernandez, M. Borjesson, T. Moragas, R. Martin, Angew. Chem., Int. Ed. 2018, 57, 15948.

[3] a) M. Costa, G. P. Chiusoli, M. Rizzardi, Chem. Commun. 1996, 27, 1699; b) M. Costa, G. P. Chiusoli, D. Taffurelli, G. Dalmonego, J. Chem. Soc. Perkin Trans. 1 1998, 1, 1541; c) R. Nicholls, S. Kaufhold, B. N Nguyen, Cat. Sci. Technol. 2014, 4, 3458; d) N.-K. Kim, H. Sogawa, K. Yamamoto, Y. Hayashi, S. Kawauchi, T. Takata, Chem. Lett. 2018, 47 1063; e) Y. Kayaki, M. Yamamoto, T. Suzuki, T. Ikariya, Green Chem. 2006, 8, 1019; f) C. Bertolotti, M. Selva, R. Maggi, E. Orlandini, G. Sartori, C. Oro, Tetrahedron Lett. 2007, 48, 2131; g) For a review, See: S. Arshadi, E. Vessally, M. Sobati, A. Hosseinian, A. Bekhradnia, J. CO2 Util. 2017, 19, 120.

[4] a) T. Mitsudo, Y. Hori, Y. Yamakawa, Y. Watanabe, Tetrahedron Lett. 1987, 28, 4417; b) M. Shi, Y. M. Shen, J. Org. Chem. 2002, 67, 16; c) P. Brunel, J. Monot, C. E. Kefalidis, L. Maron, B. Martin-Vaca, D. Bourissou, ACS Catal. 2017, 7, 2652; d) S. Yoshida, K. Fukui, S. Kikuchi, T. Yamada Chem. Lett. 2009, 38, 786; e) M. Yoshida, T. Mizuguchi, K. Shishido, Chem. Eur. J. 2012, 18, 15578; f) T. Ishida, R. Kobayashi, T. Yamada, Org. Lett. 2014, 16, 2430; g) K. Sekine, R. Kobayashi, T. Yamada, Chem. Lett. 2015, 44, 1407; h) N. Sugiyama, M. Ohseki, R. Kobayashi, K. Sekine, K. Saito, T. Yamada, Chem. Lett. 2017, 46, 1323; i) Z. Chang, X. Jing, C. He, X. Liu, C. Duan, ACS Catal. 2018, 8, 1384; j) S. Hase, Y Kayaki, T. Ikariya, ACS Catal. 2015, 5, 5135; k) K. I. Fujita, K. Inoue, J. Sato, T. Tsuchimoto, H. Yasuda, Tetrahedron 2016, 72, 1205; I) X. Liu, M. Y. Wang, S. Y. Wang, Q. Wang, L. N. He, ChemSusChem 2017, 10, 1210 ; m) M.-Y. Wang, Q.-W. Song, R. Ma, J.-N. Xie, L.-N. He, Green Chem. 2016, 18, 282.

[5] With the exception of ref. $4 \mathrm{~g}, \mathrm{~h}$, which report the formation of a C-O and a C-halogen bond.

[6] P. García-Domínguez, L. Fehr, G. Rusconi, C. Nevado, Chem. Sci. 2016 7, 3914.

[7] a) K. Benakli, C. X. Zha, R. J. Kerns, J. Am. Chem. Soc. 2001, 123, 9461 9462; b) S. Gibson, H. K. Jacobs, A. S. Gopalan, Tetrahedron Lett. 2011, 52, 887.

[8] D. A. Evans, J. Bartroli, T. L. Shih, J. Am. Chem. Soc. 1981, 103, 2127.

[9] a) D. J. Diekema, R. N. Jones, Lancet 2001, 358, 1975; b) M. R. Barbachyn, C. W. Ford, Angew. Chem., Int. Ed. 2003, 42, 2010; c) N Pandit, R. K. Singla, B. Shrivastava, Int. J. Med. Chem. 2012, 2012, 1.

[10] N. Nanbu, K. Hagiyama, M. Takehara, M. Ue, Y. Sasaki, Electrochemistry 2010, 78, 450.

[11] A. Broderick, M. A. Rocha, Y. Khalifa, M. B. Shiflett, J. T. Newberg, J. Phys. Chem. B 2019, 123, 2576.

[12] Nishikubo, T.; Kameyama, A.; Sasano, M. J. Polym. Sci. A 1994, 32, 301.

[13] B. M. Trost, E. J. McEachern, J. Am. Chem. Soc. 1999, 121, 8649.

[14] For an iodination-cross-coupling approach for the synthesis of oxazolidinone enynes, see: T. F. Tam, E. Thomas, A. Krantz, Tetrahedron Lett. 1987, 28, 1127.

[15] P. D. G. Greenwood, E. Grenet, J. Waser, Chem. Eur. J. 2019, 3010.

[16] a) U. Orcel, J. Waser, Angew. Chem., Int. Ed. 2015, 54, 5250; b) U. Orcel, J. Waser, Angew. Chem., Int. Ed. 2016, 55, 12881; c) B. Muriel, U. Orcel,
J. Waser, Org. Lett. 2017, 19, 3548; d) U. Orcel, J. Waser, Chem. Sci. 2017, 8, 32

[17] The data is available at the Cambridge Crystallographic Data Center (ccdc number: 1905359). For an example of highly stereoselective nickel-catalyzed bifunctionalization of alkynes with carbon dioxide, see: X. Wang, Y. Liu, R. Martin, J. Am. Chem. Soc. 2015, 137, 6476.

[18] R. Chinchilla, C. Nájera, Chem. Rev. 2014, 114, 1783.

[19] We thank the reviewer for highlighting this point. Potentially, the scope of the reaction could be further improved by increasing the amount of more reactive cationic palladium intermediates. In fact, carboxy-arylation with phenyl iodide was observed in about $30 \%$ yield with silver tetrafluoroborate as additive (see Supporting Information). This preliminary result is promising for further extending the scope of the methodology.

[20] a) N. Bosworth, P. Magnus, R. Moore, J. Chem. Soc., Perkin Trans. 1 1973, 2694; b) B. J. Lüssem, H.-J. Gais, J. Am. Chem. Soc. 2003, 125, 6066; c) F. D. Bobbink, W. Gruszka, M. Hulla, S. Das, P. J. Dyson, Chem. Commun. 2016, 52, 10787. 
Entry for the Table of Contents (Please choose one layout)

\section{COMMUNICATION}

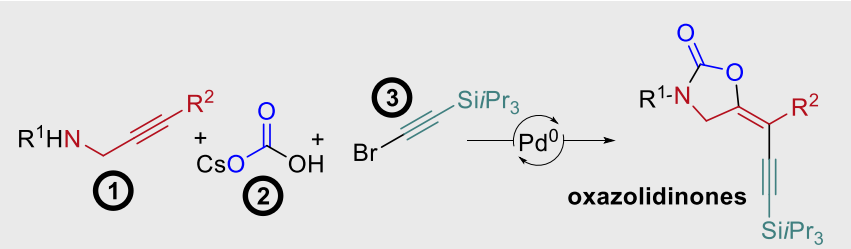

A palladium-catalyzed multi-component reaction of propargylic amines, alkynyl bromides and cesium hydrogen carbonate to access oxazolidinones is reported. In contrast to previous reports, only a slight excess of cesium hydrogen carbonate is used as surrogate of carbon dioxide.

\section{Catalysis}

Phillip D. G. Greenwood and Jerome Waser $^{*}$

Page No. - Page No.

Palladium-Catalyzed CarboxyAlkynylation of Propargylic Amines Using Carbonate Salts as Carbon Dioxide Source 


\section{Table of Contents}

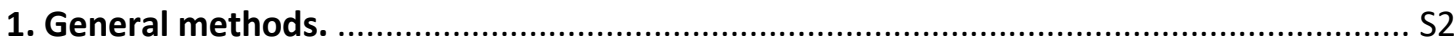

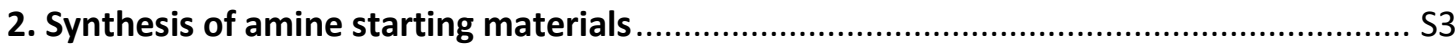

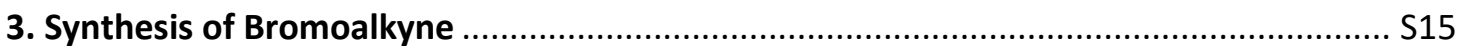

4. Optimisation of the Carbo-Oxygenation reaction. .................................................... S15

5. Scope of the Carbo-Oxygenation Reaction. ............................................................ S16

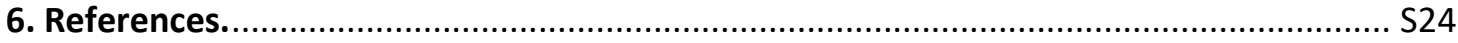

7. Structures determined by X-ray measurement. ........................................................ S25

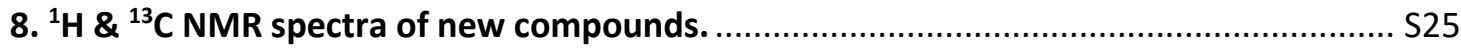

\section{General methods.}

All reactions were carried out in oven dried glassware under an atmosphere of nitrogen, unless stated otherwise. For quantitative flash chromatography technical grade solvents were used. For flash chromatography for analysis, HPLC grade solvents from Sigma-Aldrich were used. $\mathrm{THF} \mathrm{Et}_{2} \mathrm{O}, \mathrm{CH}_{3} \mathrm{CN}$, toluene, hexane and $\mathrm{CH}_{2} \mathrm{Cl}_{2}$ were dried by passage over activated alumina under nitrogen atmosphere ( $\mathrm{H}_{2} \mathrm{O}$ content $<10 \mathrm{ppm}, \mathrm{Karl}$-Fischer titration). Unless otherwise stated, degassed solvents were prepared using freeze-pump-thaw cycle. All chemicals were purchased from Acros, Aldrich, Fluka, VWR, Fluorochem or Merck and used without further purification. Chromatographic purification was performed as flash chromatography using Silicycle silica 40-63, $60 \AA$, using the solvents indicated as eluent with 0.1-0.5 bar pressure. TLC was performed on Merck silica gel 60 F254 TLC glass plates and visualized with UV light and potassium permanganate stain. ${ }^{1} \mathrm{H}-\mathrm{NMR}$ spectra were recorded on a Bruker DPX-400 $400 \mathrm{MHz}$ spectrometer in chloroform- $d$. All signals are reported in ppm with the internal chloroform signal at 7.26 $\mathrm{ppm}$. The data is being reported as ( $\mathrm{s}=$ singlet, $\mathrm{d}=$ doublet, $\mathrm{t}=$ triplet, $\mathrm{q}=$ quadruplet, $q \mathrm{i}=$ quintet, $\mathrm{m}=$ multiplet or unresolved, bs = broad signal, coupling constant(s) in $\mathrm{Hz}$, integration, assignment). ${ }^{13} \mathrm{C}-\mathrm{NMR}$ spectra were recorded with $\left\{{ }^{1} \mathrm{H}\right\}$ decoupling on a Bruker DPX-400 $100 \mathrm{MHz}$ spectrometer in chloroform-d unless otherwise stated. All signals are reported in ppm with the internal chloroform signal at $77.0 \mathrm{ppm}$ as standard unless otherwise stated. Infrared spectra were recorded on a JASCO FT-IR B4100 spectrophotometer with an ATR PRO410-S and a ZnSe prisma and are reported as $\mathrm{cm}-1$ ( $\mathrm{w}=$ weak, $\mathrm{m}=$ medium, $s=$ strong, $b r=$ broad). High resolution mass spectrometric measurements were performed by the mass spectrometry service of ISIC at the EPFL on a MICROMASS (ESI) QTOF. Cesium hydrogen carbonate (99.99\%) was purchased from AlfaAesar and stored and used under anhydrous glovebox conditions. Tris(Dibenzylideneacetone)dipalladium was purchased from flurochem and recrystallized in $200 \mathrm{mg}$ portions following reported procedure. ${ }^{1}$ DPEPhos was purchased from Acros. Deactivated silica gel was prepared by making a slurry of silica gel (230-400mesh) with $5 \% \mathrm{Et}_{3} \mathrm{~N}$ in pentane solution followed by complete removal of solvent by rotary evaporation until it is a free flowing powder. The solvent system ULTRA comprises $71 \%$ DCM, $24 \%$ methanol and $5 \%$ aq. ammonia ( $25 \%$ solution). 


\section{Synthesis of amine starting materials}

\section{N-Benzyl but-2-ynylamine (2a)}

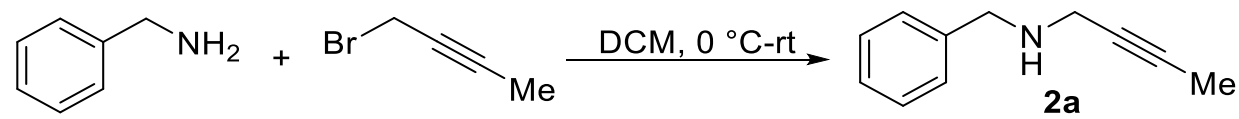

A solution of benzyl amine (4-6 equiv.) in DCM (15 mL) at $0{ }^{\circ} \mathrm{C}$ was stirred vigorously while a solution of bromo-2-butyne (2.5 mL, $27 \mathrm{mmol}, 1$ equiv.) in DCM $(15 \mathrm{~mL})$ was slowly added. The reaction mixture was then warmed to room temperature and stirred for 5 hours. It was then filtered through silica gel, eluting with 40\% EtOAc in pentane and the resulting solution concentrated. Purification was performed by column chromatography $\left(\mathrm{SiO}_{2}, 10-40 \%\right.$ EtOAc in pentane) to afford benzyl butynylamine (2a) as a straw yellow oil (3.4g, $21 \mathrm{mmol}, 74 \%$ yield). Further purification could be achieved by Kugelrohr distillation $\left(86^{\circ} \mathrm{C}\right.$ at $5 \times 10^{-}$ $\left.{ }^{1} \mathrm{mbar}\right)$.

${ }^{1} \mathrm{H}$ NMR (400 MHz, Chloroform-d) $\delta 7.39-7.21(\mathrm{~m}, 5 \mathrm{H}, \mathrm{ArH}), 3.86\left(\mathrm{~s}, 2 \mathrm{H}, \mathrm{ArCH}_{2}\right), 3.38(\mathrm{q}, \mathrm{J}=2.4 \mathrm{~Hz}, 2 \mathrm{H}$, $\left.\mathrm{CH}_{2} \mathrm{C} \equiv \mathrm{C}\right), 1.85\left(\mathrm{t}, \mathrm{J}=2.4 \mathrm{~Hz}, 3 \mathrm{H}, \mathrm{CH}_{3}\right), 1.57$ (bs, $1 \mathrm{H} \mathrm{NH}$ ).

${ }^{13} \mathrm{C}$ NMR (101 MHz, Chloroform-d) $\delta$ 139.7, 128.4, 128.3, 127.0, 79.1, 77.1, 52.5, 37.8, 3.5.

Spectra data was consistent with the values reported in literature. ${ }^{2}$

\section{$\underline{\mathrm{N}-(4-m e t h o x y b e n z y l) b u t-2-y n-1-a m i n e ~(1 b)}$}

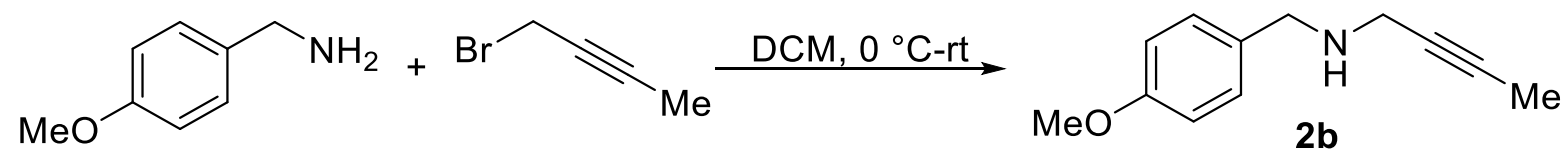

A solution of 4-methoxy benzyl amine (6.3 g, $48 \mathrm{mmol}, 5$ equiv.) in $\mathrm{DCM}(15 \mathrm{~mL})$ at $0{ }^{\circ} \mathrm{C}$ was stirred vigorously while a solution of bromo-2-butyne (1.2 g, $9.1 \mathrm{mmol}, 1$ equiv.) in DCM (15 mL) was slowly added over a period of 10 minutes. After complete addition, the reaction mixture was allowed to warm to room temperature and stirring continued for 5 hours. It was then filtered through silica gel eluting with $40 \%$ EtOAc in pentane and the resulting solution concentrated. Purification was performed by column chromatography $\left(\mathrm{SiO}_{2}, 10-40 \%\right.$ EtOAc in pentane) to afford N-(4-methoxybenzyl)but-2-yn-1-amine (2b) as a straw yellow oil ( $1.0 \mathrm{~g}, 5.3 \mathrm{mmol}, 60 \%$ yield).

${ }^{1} \mathrm{H}$ NMR $\left(400 \mathrm{MHz}\right.$, Chloroform-d) $\delta 7.32-7.24(\mathrm{~m}, 2 \mathrm{H}, \operatorname{ArH}), 6.90-6.84(\mathrm{~m}, 2 \mathrm{H}, \operatorname{ArH}), 3.81(\mathrm{~s}, 2 \mathrm{H}, \operatorname{ArCH})_{2}$, $3.80\left(\mathrm{~s}, 3 \mathrm{H}, \mathrm{OCH}_{3}\right), 3.37\left(\mathrm{dq}, J=3.4,2.1 \mathrm{~Hz}, 2 \mathrm{H}, \mathrm{CH}_{2} \mathrm{C} \equiv \mathrm{C}\right), 1.85$ (dd, $J=2.8,1.9 \mathrm{~Hz}, 3 \mathrm{H}, \mathrm{CH}_{3}$ ).

${ }^{13} \mathrm{C}$ NMR (101 MHz, Chloroform-d) $\delta$ 158.6, 131.8, 129.5, 113.7, 79.1, 77.2, 55.2, 51.9, 37.7, 3.5.

HRMS (ESI/QTOF) m/z: [M + H] Calcd for $\mathrm{C}_{12} \mathrm{H}_{16} \mathrm{NO}^{+}$190.1226; Found 190.1225.

\section{Ferrocenylmethyl-but-2-ynylamine (6)}

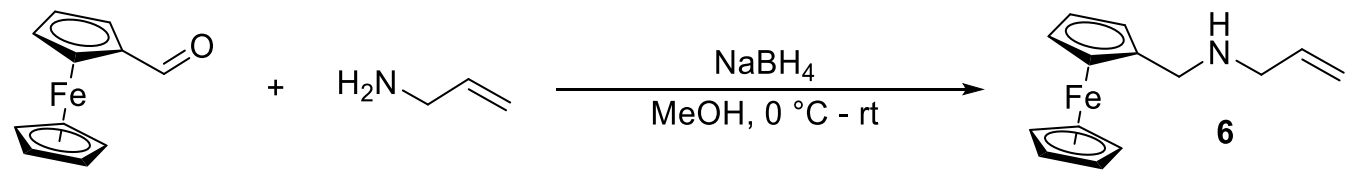


A solution of ferrocenecarboxaldehyde $(3.3 \mathrm{~g}, 15 \mathrm{mmol}, 1$ equiv) and allylamine $(0.97 \mathrm{~g}, 17 \mathrm{mmol}, 1.1$ equiv.) in methanol ( $66 \mathrm{~mL}$ ) was stirred at room temperature for 30 minutes before the reaction mixture was cooled to $0{ }^{\circ} \mathrm{C}$ by an ice bath. $\mathrm{NaBH}_{4}(700 \mathrm{mg}, 18.5 \mathrm{mmol}, 1.2$ equiv.) was carefully added and the reaction mixture was stirred for a further 20 minutes before it was warmed to room temperature for 4 hours. The reaction was diluted with EtOAc $(30 \mathrm{~mL})$ and the organic layer washed with saturated $\mathrm{NaHCO}_{3}$ solution $(30 \mathrm{~mL})$. The aqueous layer was washed with additional EtOAc $(2 \times 30 \mathrm{~mL})$. The combined organic portions were washed with brine $(30 \mathrm{~mL})$, dried over $\mathrm{MgSO}_{4}$, filtered and concentrated. To the crude material was added pentane $(100 \mathrm{~mL})$ causing precipitation of solid which was filtered off to afford ferrocenylmethyl allylamine (6) as a brown solid (3.7 g, 14, 93\% yield) which was sufficiently pure to be used without further purification.

${ }^{1} \mathrm{H}$ NMR $\left(400 \mathrm{MHz}\right.$, Chloroform-d) $\delta 5.92$ (ddt, $J=17.2,10.3,6.0 \mathrm{~Hz}, 1 \mathrm{H}, \mathrm{CH}=\mathrm{CH}_{2}$ ), 5.19 (dq, J=17.2, $1.7 \mathrm{~Hz}$, $\left.1 \mathrm{H}, \mathrm{CH}=\mathrm{CH}_{2}\right), 5.11\left(\mathrm{dq}, J=10.2,1.4 \mathrm{~Hz}, 1 \mathrm{H}, \mathrm{CH}=\mathrm{CH}_{2}\right), 4.22-4.07(\mathrm{~m}, 9 \mathrm{H}$, ferrocene $H), 3.51(\mathrm{~s}, 2 \mathrm{H}$, ferrocene $\left.\mathrm{CH}_{2}\right), 3.28\left(\mathrm{dt}, J=6.0,1.4 \mathrm{~Hz}, 2 \mathrm{H}, \mathrm{CH}_{3}\right)$.

Spectra data was consistent with the values reported in literature. ${ }^{3}$

\section{Ferrocenylmethyl-but-2-ynylamine (2c)}
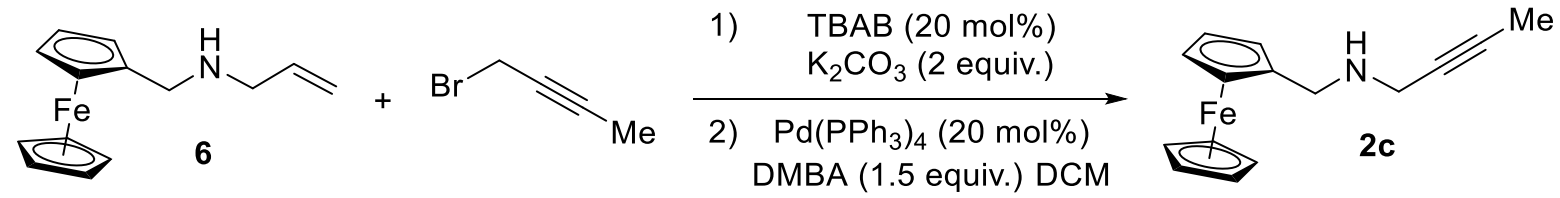

To a solution of Ferrocenylmethyl allylamine (6) (0.65 g, $2.5 \mathrm{mmol}, 1$ equiv.) in MeCN (12 mL) was added bromobutyne $\left(0.24 \mathrm{~mL}, 2.8 \mathrm{mmol}, 1.1\right.$ equiv.) TBAB $(0.16 \mathrm{~g}, 0.51 \mathrm{mmol}, 20 \mathrm{~mol} \%)$ and $\mathrm{K}_{2} \mathrm{CO}_{3}(0.70 \mathrm{~g}, 5.1$ mmol, 2 equiv.). The reaction mixture was heated to $55^{\circ} \mathrm{C}$ and left to stir over night. It was then filtered through a short pad of Celite, which was washed with EtOAc and the filtrate concentrated under reduced pressure. The crude material was dry loaded onto $\mathrm{SiO}_{2}$ and purified by column chromatography $\left(\mathrm{SiO}_{2}, 5-\right.$ $15 \%$ EtOAc in pentane) to afford $\mathrm{N}$-allyl-N-Ferrocenylmethyl-but-2-yn-1-amine as a dark orange oil (0.49 $\mathrm{g}$ , $1.6 \mathrm{mmol}, 62 \%$ yield)

${ }^{1} \mathrm{H}$ NMR (400 MHz, Chloroform-d) $\delta 5.83$ (ddt, $J=16.9,10.2,6.6 \mathrm{~Hz}, 1 \mathrm{H}, \mathrm{CH}=\mathrm{CH}_{2}$ ), 5.23 (dq, J=17.2, $1.6 \mathrm{~Hz}$, $\left.1 \mathrm{H}, \mathrm{CH}=\mathrm{CH}_{2}\right), 5.15\left(\mathrm{ddt}, \mathrm{J}=10.1,2.2,1.2 \mathrm{~Hz}, 1 \mathrm{H}, \mathrm{CH}=\mathrm{CH}_{2}\right), 4.24-4.07(\mathrm{~m}, 9 \mathrm{H}$, ferroceneH), $3.47(\mathrm{~s}, 2 \mathrm{H}$, ferroceneCH $\mathrm{C}_{2}$ ), $3.20\left(\mathrm{q}, J=2.3 \mathrm{~Hz}, 2 \mathrm{H}, \mathrm{NCH}_{2} \mathrm{C} \equiv \mathrm{C}\right.$ ), $3.08\left(\mathrm{dt}, J=6.6,1.4 \mathrm{~Hz}, 2 \mathrm{H}, \mathrm{NCH}_{2} \mathrm{CHCH}_{2}\right.$ ), $1.88(\mathrm{t}, J=2.4$ $\mathrm{Hz}, 3 \mathrm{H}, \mathrm{CH}_{3}$ ).

${ }^{13} \mathrm{C}$ NMR $\left(101 \mathrm{MHz}, \mathrm{CDCl}_{3}\right) \delta$ 135.9, 117.7, 83.0, 80.6, 74.0, 70.1, 68.5, 68.0, 56.4, 52.3, 41.4, 3.6.

IR (cm $\left.{ }^{-1}\right) 3087(\mathrm{~m}), 2920(\mathrm{~m}), 2811(\mathrm{~m}), 1645(\mathrm{~m}), 1431(\mathrm{~m}), 1330(\mathrm{~m}), 1107(\mathrm{~s}), 1000(\mathrm{~s}), 922(\mathrm{~s}), 819(\mathrm{~s})$

HRMS (APCI/QTOF) m/z: [M] $]^{+}$Calcd for $\mathrm{C}_{18} \mathrm{H}_{21} \mathrm{FeN}^{+}$307.1018; Found 307.1025.

The tertiary amine obtained from the previous step ( $0.45 \mathrm{~g}, 1.5 \mathrm{mmol}, 1$ equiv.) was added to a solution of $\mathrm{Pd}\left(\mathrm{PPh}_{3}\right)_{4}$ (34 mg, $29 \mu \mathrm{mol}, 2 \mathrm{~mol} \%$ ) and 1,3-dimethylbarbituric acid (0.34 g, $2.2 \mathrm{mmol}, 1.5$ equiv.) in DCM $(10 \mathrm{~mL})$ under an $\mathrm{N}_{2}$ atmosphere. The reaction mixture was stirred at room temperature for 6 hours. Reaction completion was checked by TLC ( $2 \%$ EtOAc in pentane). The reaction mixture was diluted with ether $(40 \mathrm{~mL})$ and washed with sat. $\mathrm{NaHCO}_{3}(3 \times 15 \mathrm{~mL})$. The organic layer was extracted with aq. $\mathrm{HCl}(1.0$ $\mathrm{M} ; 3 \times 15 \mathrm{~mL}$ ) after which the combined aqueous layers and any precipitated solids were basified with 
$\mathrm{K}_{2} \mathrm{CO}_{3}(\mathrm{pH}>7)$ and extracted with DCM $(3 \times 20 \mathrm{~mL})$. The combined extracts were dried over $\mathrm{MgSO}_{4}$, filtered and concentrated by rotary evaporation. The collected crude product was sufficiently pure to be used without any further purification. Ferrocenylmethyl-but-2-ynylamine (2c) was obtained as a dark brown oil (0.37 g, $1.4 \mathrm{mmol}, 94 \%$ yield)

${ }^{1} \mathrm{H}$ NMR (400 MHz, Chloroform-d) $\delta 4.22-4.09$ (m, 9H, ferroceneH), 3.57 (s, 2H, ferroceneCH $\left.\mathrm{CH}_{2}\right), 3.39$ (q, $J$ $\left.=2.4 \mathrm{~Hz}, 2 \mathrm{H}, \mathrm{NCH}_{2} \mathrm{C} \equiv \mathrm{C}\right), 1.85\left(\mathrm{t}, J=2.4 \mathrm{~Hz}, 3 \mathrm{H}, \mathrm{CH}_{3}\right)$.

${ }^{13} \mathrm{C}$ NMR (101 MHz, Chloroform-d) $\delta 86.0,79.1,68.4,68.3,67.8,47.4,37.9,3.5$.

HRMS (ESI/QTOF) m/z: [M] $]^{+}$Calcd for $\mathrm{C}_{15} \mathrm{H}_{17} \mathrm{FeN}^{+}$267.0705; Found 267.0706.

\section{N-Benzyl 4-phenyl-but-2-ynylamine (2d)}

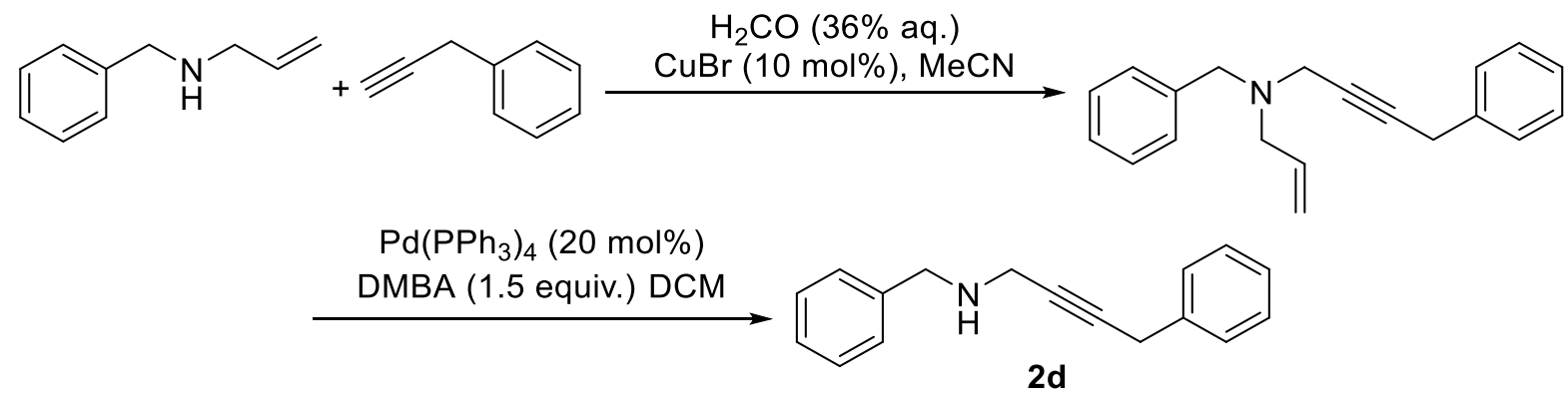

Following an adapted version of a reported procedure. ${ }^{2}$ To a solution of $\mathrm{CuBr}(0.18 \mathrm{~g}, 1.3 \mathrm{mmol}, 12 \mathrm{~mol} \%)$ in $\mathrm{MeCN}(60 \mathrm{~mL}$ ) was added allyl benzylamine (1.9 g, $13 \mathrm{mmol}, 1.3$ equiv), formaldehyde $(2.5 \mathrm{~mL}, 33 \mathrm{mmol}$ $36 \%$ aq. solution, 3.1 equiv) and phenylpropyne ( $1.2 \mathrm{~g}, 10 \mathrm{mmol}, 1$ equiv.). The reaction mixture was stirred at room temperature for 16 hours after which it was concentrated by rotary evaporation. The residue was diluted with $\mathrm{Et}_{2} \mathrm{O}(20 \mathrm{~mL})$ and washed with aq. $\mathrm{NaOH}$ solution $(5.0 \mathrm{M}, 3 \times 10 \mathrm{~mL})$, dried over $\mathrm{MgSO}_{4}$, filtered and concentrated by rotary evaporation. Purification of the crude product by column chromatography $\left(\mathrm{SiO}_{2}, 0-2 \%\right.$ EtOAc in pentane) to afford N-allyl-N-benzyl-4-phenyl-but-2-ynylamine as a colourless oil (2.6 g, $9.3 \mathrm{mmol}, 89 \%$ yield)

${ }^{1} \mathrm{H}$ NMR (400 MHz, Chloroform-d) $\delta 7.49-7.28$ (m, 10H, ArH), 5.97 (dddd, J = 17.3, 12.4, 6.5, $2.3 \mathrm{~Hz}, 1 \mathrm{H}$, $\left.\mathrm{CH}=\mathrm{CH}_{2}\right), 5.34\left(\mathrm{dq}, J=17.2,1.7 \mathrm{~Hz}, 1 \mathrm{H}, \mathrm{CH}=\mathrm{CH}_{2}\right), 5.24\left(\mathrm{dq}, J=10.4,1.6 \mathrm{~Hz}, 1 \mathrm{H}, \mathrm{CH}=\mathrm{CH}_{2}\right), 3.79-3.73(\mathrm{~m}, 4 \mathrm{H}$, $\mathrm{ArCH}_{2} \mathrm{~N}, \mathrm{CH}_{2} \mathrm{CH}=\mathrm{CH}_{2}$ ), 3.44 (q, $J=2.5 \mathrm{~Hz}, 2 \mathrm{H}, \mathrm{C} \equiv \mathrm{CCH}_{2} \mathrm{Ph}$ ), $3.27\left(\mathrm{dt}, J=6.6,1.6 \mathrm{~Hz}, 2 \mathrm{H}, \mathrm{NCH}_{2} \mathrm{C} \equiv \mathrm{C}\right.$ ).

${ }^{13} \mathrm{C}$ NMR (101 MHz, Chloroform-d) $\delta 138.7,137.1,135.7,129.1,128.4,128.2,127.8,127.0,126.5,117.8$, $82.9,77.0,57.3,56.7,41.8,25.1$.

The tertiary amine obtained from the previous step $(1.0 \mathrm{~g}, 3.6 \mathrm{mmol}, 1$ equiv.) was added to a solution of $\mathrm{Pd}\left(\mathrm{PPh}_{3}\right)_{4}(84 \mathrm{mg}, 73 \mu \mathrm{mol}, 2 \mathrm{~mol} \%$ ) and 1,3-dimethylbarbituric acid ( $0.85 \mathrm{~g}, 5.5 \mathrm{mmol}, 1.5$ equiv.) in DCM $(22 \mathrm{~mL})$ under an $\mathrm{N}_{2}$ atmosphere. The reaction mixture was stirred at room temperature for 6 hours. Reaction completion was checked by TLC ( $2 \%$ EtOAc in pentane). The reaction mixture was diluted with ether $(40 \mathrm{~mL})$ and washed with sat. $\mathrm{NaHCO}_{3}(3 \times 15 \mathrm{~mL})$. The organic layer was extracted with aq. $\mathrm{HCl}(1.0$ $\mathrm{M} ; 3 \times 15 \mathrm{~mL}$ ) after which the combined aqueous layers and any precipitated solids were basified with $\mathrm{K}_{2} \mathrm{CO}_{3}(\mathrm{pH}>7)$ and extracted with DCM $(3 \times 25 \mathrm{~mL})$. The combined extracts were dried over $\mathrm{MgSO}_{4}$, filtered and concentrated by rotary evaporation. Purification by flash column chromatography $\left(\mathrm{SiO}_{2}, 20-30 \%\right.$ 
EtOAc in pentane) to afford N-benzyl-4-phenyl-but-2-ynylamine (2d) as a straw coloured oil (0.76 g, 3.0 $\mathrm{mmol}, 83 \%$ yield)

${ }^{1} \mathrm{H}$ NMR $\left(400 \mathrm{MHz}\right.$, Chloroform-d) $\delta 7.41-7.20(\mathrm{~m}, 10 \mathrm{H}, \mathrm{ArH}), 3.90\left(\mathrm{~s}, 2 \mathrm{H}, \operatorname{ArCH}_{2} \mathrm{~N}\right), 3.65(\mathrm{t}, J=2.3 \mathrm{~Hz}, 2 \mathrm{H}$, $\left.\mathrm{C} \equiv \mathrm{CCH}_{2} \mathrm{Ph}\right), 3.48\left(\mathrm{t}, J=2.3 \mathrm{~Hz}, 2 \mathrm{H}, \mathrm{NCH}_{2} \mathrm{C} \equiv \mathrm{C}\right), 1.65(\mathrm{bs}, 1 \mathrm{H}, \mathrm{NH})$.

${ }^{13} \mathrm{C}$ NMR (101 MHz, Chloroform-d) $\delta$ 139.5, 137.0, 128.5, 128.4 (2C), 127.9, 127.1, 126.6, 81.4, 80.2, 52.5, $37.9,25.2$.

IR (cm-1) 3025 (s), 3063 (s), 2919 (s), 2838 (s), 1606 (s), 1496 (s), 1454 (s), 728 (s), 695 (s)

HRMS (ESI/QTOF) m/z: [M+ H] Calcd for $\mathrm{C}_{17} \mathrm{H}_{18} \mathrm{~N}^{+}$236.1434; Found 236.1436.

Spectra data was consistent with the values reported in literature. ${ }^{4}$

\section{N-Benzyl 3-cyclopropyl-prop-2-ynylamine (2e)}
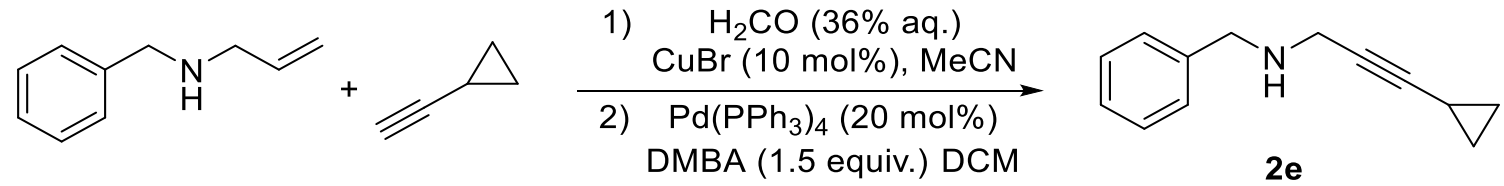

Following an adapted version of a reported procedure. ${ }^{2}$ To a solution of $\mathrm{CuBr}(0.36 \mathrm{~g}, 2.5 \mathrm{mmol}, 12 \mathrm{~mol} \%)$ in MeCN (130 mL) was added allyl benzylamine ( $3.9 \mathrm{~mL}, 25 \mathrm{mmol}, 1.3$ equiv), formaldehyde ( $36 \%$ aq. solution; $5.0 \mathrm{~mL}, 65 \mathrm{mmol}, 3.3$ equiv.) and ethenylcyclopropane (1.7 mL, $20 \mathrm{mmol}, 1$ equiv.). The reaction was stirred at room temperature for 16 hours before being concentrated by rotary evaporation. The resulting residue was diluted with $\mathrm{Et}_{2} \mathrm{O}(20 \mathrm{~mL})$ and washed with aq. $\mathrm{NaOH}(5.0 \mathrm{M} ; 3 \times 10 \mathrm{~mL})$, dried over $\mathrm{MgSO}_{4}$, filtered and concentrated by rotary evaporation. Purification of the crude material by column chromatography $\left(\mathrm{SiO}_{2}, 0-2 \%\right.$ EtOAc in pentane) afforded $\mathrm{N}$-allyl-N-benzyl 3-cyclopropyl-prop-2-ynylamine as a colourless oil $(4.0 \mathrm{~g}, 18 \mathrm{mmol}, 89 \%$ yield).

${ }^{1} \mathrm{H}$ NMR $\left(400 \mathrm{MHz}\right.$, Chloroform-d) $\delta 7.43-7.19(\mathrm{~m}, 5 \mathrm{H}, \mathrm{ArH}), 5.88\left(\mathrm{ddt}, \mathrm{J}=16.8,10.1,6.5 \mathrm{~Hz}, 1 \mathrm{H}, \mathrm{CH}=\mathrm{CH}_{2}\right)$, $5.25\left(\mathrm{dq}, J=17.2,1.6 \mathrm{~Hz}, 1 \mathrm{H}, \mathrm{CH}=\mathrm{CH}_{2}\right), 5.16\left(\mathrm{ddt}, J=10.1,2.2,1.2 \mathrm{~Hz}, 1 \mathrm{H}, \mathrm{CH}=\mathrm{CH}_{2}\right), 3.61(\mathrm{~s}, 2 \mathrm{H}, \operatorname{ArCH})_{2}$, 3.24 (d, J = 1.9 Hz, 2H, CH $\mathrm{C}_{2}=\mathrm{C}$ ), 3.14 (dt, J=6.5, $1.3 \mathrm{~Hz}, 2 \mathrm{H}, \mathrm{CH}_{2} \mathrm{C}=\mathrm{C}$ ), 1.28 (dddd, J=10.1, 8.6, 5.0, $2.5 \mathrm{~Hz}$, $1 \mathrm{H}, \mathrm{CH}$ cyclopropane), $0.81-0.75$ (m, 2H, $\mathrm{CH}_{2}$ cyclopropane), $0.70-0.66$ (m, 2H, $\mathrm{CH}_{2}$ cyclopropane).

${ }^{13} \mathrm{C}$ NMR (101 MHz, Chloroform-d) $\delta 138.7,135.8,129.2,128.2,127.0,117.8,88.9,69.8,57.2,56.6,41.8$, $8.3,-0.5$.

Under a nitrogen atmosphere, the tertiary amine obtained from the previous step $(1.0 \mathrm{~g}, 4.4 \mathrm{mmol}, 1.0$ equiv.) was added to a solution of $\mathrm{Pd}\left(\mathrm{PPh}_{3}\right)_{4}(0.10 \mathrm{~g}, 89 \mu \mathrm{mol}, 2 \mathrm{~mol} \%)$ and 1,3-dimethylbarbituric acid (1.0 $\mathrm{g}, 6.7 \mathrm{mmol}, 1.5$ equiv.) in $\mathrm{DCM}(22 \mathrm{~mL})$. The reaction mixture was stirred at room temperature for 6 hours. The mixture was then diluted with $\mathrm{Et}_{2} \mathrm{O}(50 \mathrm{~mL})$, washed with sat. aq. $\mathrm{NaHCO}_{3}(3 \times 20 \mathrm{~mL})$ and then extracted with $1 \mathrm{M} \mathrm{HCl}(3 \times 20 \mathrm{~mL})$. The combined aqueous layers were basified with $\mathrm{K}_{2} \mathrm{CO}_{3}$ (until pH >7) and then extracted with DCM $(3 \times 20 \mathrm{~mL})$ after which the combined organic layers were dried over $\mathrm{MgSO}_{4}$, filtered and concentrated by rotary evaporation. The crude material was purified by column chromatography $\left(\mathrm{SiO}_{3}, 20-30 \%\right.$ EtOAc in pentane) to afford N-benzyl 3-cyclopropyl-prop-2-ynylamine (2e) as a lightly straw coloured oil ( $0.82 \mathrm{~g}, 4.4 \mathrm{mmol}, 99 \%$ yield). 
${ }^{1} \mathrm{H}$ NMR (400 MHz, Chloroform-d) $\delta 7.45-7.19(\mathrm{~m}, 5 \mathrm{H}, \mathrm{ArH}), 3.84\left(\mathrm{~s}, 2 \mathrm{H}, \mathrm{ArCH}_{2}\right), 3 \mathrm{z} \mathrm{Hz}, 1 \mathrm{H}, \mathrm{CH}$ cyclopropane), $0.80-0.63$ ( $\mathrm{m}, 4 \mathrm{H}, \mathrm{CH}_{2}$ cyclopropane).

${ }^{13} \mathrm{C}$ NMR (101 MHz, Chloroform-d) $\delta$ 139.7, 128.4 (2C), 127.0, 87.0, 73.3, 52.5, 37.9, 8.1, -0.5.

Spectra data was consistent with the values reported in literature. ${ }^{2}$

\section{N-Benzyl 4,4-dimethyl-pent-2-ynylamine (2f)}

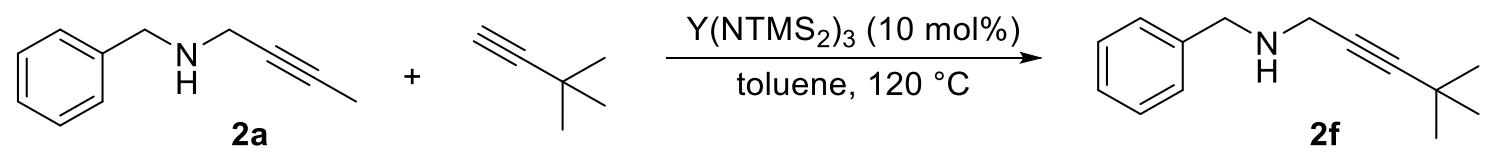

Into an oven dried microwave vial containing a stirring bar was weighed $\left.\mathrm{Y}\left(\mathrm{N}(\mathrm{TMS})_{2}\right)_{3}\right)(0.11 \mathrm{~g}, 0.20 \mathrm{mmol}$, $10 \mathrm{~mol} \%$ ). Dry toluene (degassed; $13 \mathrm{~mL}$ ), N-Benzyl but-2-ynylylamine (2a) (0.32 g, $2.0 \mathrm{mmol}, 1$ equiv.) and 3,3-dimethylbytyne $\left(0.66 \mathrm{~g}, 8.0 \mathrm{mmol}, 4\right.$ equiv.) were added. The reaction mixture was stirred at $130{ }^{\circ} \mathrm{C}$ for 24 hours, after which, the reaction mixture was cooled to room temperature, and was loaded directly onto silica for purification by column chromatography $\left(\mathrm{SiO}_{2}, 5: 5: 90-5: 10: 85\right.$ Ultra and EtOAc in pentane), which afforded N-benzyl 4,4-dimethyl-pent-2-ynylamine (2f) as a straw yellow oil $(0.27 \mathrm{~g}, 1.3 \mathrm{mmol}, 66 \%$ yield).

${ }^{1} \mathrm{H}$ NMR (400 MHz, Chloroform-d) $\delta 7.39-7.21(\mathrm{~m}, 5 \mathrm{H}, \mathrm{ArH}), 3.87\left(\mathrm{~s}, 2 \mathrm{H}, \mathrm{ArCH}_{2}\right), 3.40\left(\mathrm{~s}, 2 \mathrm{H}, \mathrm{CH} \mathrm{C}_{2} \equiv \mathrm{C}\right), 1.88$ (bs, $1 \mathrm{H}, \mathrm{NH}), 1.24\left(\mathrm{~s}, 9 \mathrm{H}, \mathrm{C}\left(\mathrm{CH}_{3}\right)_{3}\right)$.

${ }^{13} \mathrm{C}$ NMR (101 MHz, Chloroform-d) $\delta 139.4,128.5,128.4,127.1,92.8,77.2,52.2,37.7,31.2,27.4$.

Spectra data was consistent with the values reported in literature. ${ }^{2}$

\section{N-benzyl-3-phenylprop-2-yn-1-amine (2g)}

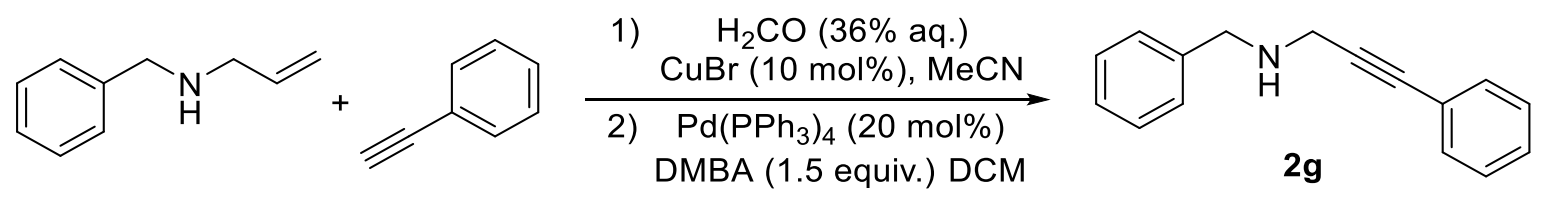

Following an adapted version of a reported procedure. ${ }^{2}$ To a solution of $\mathrm{CuBr}(0.20 \mathrm{~g}, 1.4 \mathrm{mmol}, 12 \mathrm{~mol} \%)$ in $\mathrm{MeCN}(70 \mathrm{~mL})$ was added allyl benzylamine $(2.0 \mathrm{~g}, 14 \mathrm{mmol}, 1.3$ equiv.), formaldehyde $(2.5 \mathrm{~mL}, 33 \mathrm{mmol}$ $36 \%$ aq. solution, 3 equiv.) and phenylpropyne ( $1.2 \mathrm{~g}, 11 \mathrm{mmol}, 1$ equiv.). The reaction mixture was stirred at room temperature for 16 hours, after which it was concentrated by rotary evaporation. The residue was diluted with $\mathrm{Et}_{2} \mathrm{O}(20 \mathrm{~mL})$ and washed with aq. $\mathrm{NaOH}$ solution $(5.0 \mathrm{M} ; 3 \times 10 \mathrm{~mL})$, dried over $\mathrm{MgSO}_{4}$, filtered and concentrated by rotary evaporation. Purification of the crude product by column chromatography $\left(\mathrm{SiO}_{2}, 0-2 \%\right.$ EtOAc in pentane) to afford $\mathrm{N}$-allyl-N-methyl-4-phenyl-but-2-ynylamine as a colourless oil (2.7 g, $10.3 \mathrm{mmol}, 95 \%$ yield)

${ }^{1} \mathrm{H}$ NMR (400 MHz, Chloroform-d) $\delta 7.52-7.22(\mathrm{~m}, 10 \mathrm{H}, \mathrm{ArH}), 5.93$ (ddt, $J=16.7,10.1,6.5 \mathrm{~Hz}, 1 \mathrm{H}, \mathrm{CH}=\mathrm{CH}_{2}$ ), $5.31\left(\mathrm{dq}, J=17.1,1.6 \mathrm{~Hz}, 1 \mathrm{H}, \mathrm{CH}=\mathrm{CH}_{2}\right), 5.20$ (ddt, $\left.J=10.1,2.2,1.2 \mathrm{~Hz}, 1 \mathrm{H}, \mathrm{CH}=\mathrm{CH}_{2}\right), 3.72(\mathrm{~s}, 2 \mathrm{H}, \operatorname{ArCH})_{2}$, $3.53\left(\mathrm{~s}, 2 \mathrm{H}, \mathrm{CH}_{2} \mathrm{C} \equiv \mathrm{C}\right), 3.26\left(\mathrm{dt}, J=6.5,1.4 \mathrm{~Hz}, 2 \mathrm{H}, 2 \mathrm{H}, \mathrm{CH}_{2} \mathrm{C}=\mathrm{C}\right)$. 
${ }^{13} \mathrm{C}$ NMR (101 MHz, Chloroform-d) $\delta$ 138.7, 135.7, 131.7, 129.2, 128.3, 128.3, 128.0, 127.1, 123.4, 118.0, 85.7, 84.4, 57.4, 56.8, 42.1 .

The tertiary amine obtained from the previous step ( $1.5 \mathrm{~g}, 5.7 \mathrm{mmol}, 1$ equiv.) was added to a solution of $\mathrm{Pd}\left(\mathrm{PPh}_{3}\right)_{4}(0.13 \mathrm{~g}, 0.12 \mathrm{~mol}, 2 \mathrm{~mol} \%$ ) and 1,3-dimethylbarbituric acid (1.3 g, $8.6 \mathrm{mmol}, 1.5$ equiv.) in DCM $(35 \mathrm{~mL})$ under an $\mathrm{N}_{2}$ atmosphere. The reaction mixture was stirred at room temperature for 6 hours. Reaction completion was checked by TLC ( $20 \%$ EtOAc in pentane). The reaction mixture was concentrated to a quarter of its original volume and diluted with ether $(50 \mathrm{~mL})$ and washed with sat. $\mathrm{NaHCO}_{3}(3 \times 15$ $\mathrm{mL})$. The organic layer was extracted with aq. $\mathrm{HCl}(1.0 \mathrm{M} ; 3 \times 15 \mathrm{~mL})$ after which the combined aqueous layers and precipitated solids were basified with $\mathrm{K}_{2} \mathrm{CO}_{3}(\mathrm{pH}>7)$ and extracted with DCM $(3 \times 25 \mathrm{~mL})$. The combined extracts were dried over $\mathrm{MgSO}_{4}$, filtered and concentrated by rotary evaporation. Purification by flash column chromatography $\left(\mathrm{SiO}_{2}, 10-30 \%\right.$ EtOAc in pentane) to afford N-benzyl-3-phenylprop-2-yn1 -amine $(2 \mathrm{~g})$ as a straw yellow oil $(1.1 \mathrm{~g}, 5.0 \mathrm{mmol}, 87 \%$ yield)

${ }^{1} \mathrm{H}$ NMR (400 MHz, Chloroform-d) $\delta 7.52-7.20(\mathrm{~m}, 10 \mathrm{H}, \mathrm{ArH}), 3.96\left(\mathrm{~s}, 2 \mathrm{H}, \mathrm{ArCH}_{2}\right), 3.66\left(\mathrm{~s}, 2 \mathrm{H}, \mathrm{CH}_{2} \mathrm{C} \equiv \mathrm{C}\right)$, 1.73 (bs, $1 \mathrm{H}, \mathrm{NH})$.

${ }^{13} \mathrm{C}$ NMR (101 MHz, Chloroform-d) $\delta 139.5,131.7,128.5$ (2C), 128.3, 128.1, 127.2, 123.2, 87.5, 83.8, 52.5, 38.3.

Spectra data was consistent with the values reported in literature. ${ }^{2}$

\section{N-Methyl 4-phenyl-but-2-ynylamine (2h)}

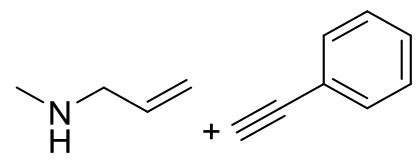

$$
\text { 1) } \begin{gathered}
\mathrm{H}_{2} \mathrm{CO}(36 \% \text { aq. }) \\
\mathrm{CuBr}(10 \mathrm{~mol} \%), \mathrm{MeCN}
\end{gathered}
$$

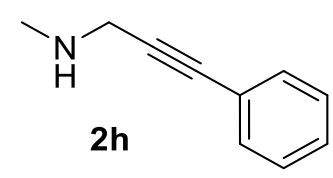

Following an adapted version of a reported procedure. ${ }^{2} \mathrm{To}$ a solution of $\mathrm{CuBr}(0.20 \mathrm{~g}, 1.4 \mathrm{mmol}, 13 \mathrm{~mol} \%)$ in $\mathrm{MeCN}(70 \mathrm{~mL})$ was added allyl methylamine $(0.98 \mathrm{~g}, 14 \mathrm{mmol}, 1.3$ equiv.), formaldehyde $(2.5 \mathrm{~mL}, 33$ $\mathrm{mmol} 36 \%$ aq. solution, 3 equiv.) and phenylpropyne (1.2 $\mathrm{g}, 11 \mathrm{mmol}, 1$ equiv.). The reaction mixture was stirred at room temperature for 16 hours after which it was concentrated by rotary evaporation. The residue was diluted with $\mathrm{Et}_{2} \mathrm{O}(20 \mathrm{~mL})$ and washed with aq. $\mathrm{NaOH}$ solution $(5.0 \mathrm{M} ; 3 \times 10 \mathrm{~mL})$, dried over $\mathrm{MgSO}_{4}$, filtered and concentrated by rotary evaporation. Purification of the crude product by column chromatography ( $\mathrm{SiO}_{2}, 0-2 \%$ EtOAc in pentane) to afford $\mathrm{N}$-allyl-N-benzyl-4-phenyl-but-2-ynylamine as a colourless oil ( $1.8 \mathrm{~g}, 9.7 \mathrm{mmol}, 88 \%$ yield)

${ }^{1} \mathrm{H}$ NMR (400 MHz, Chloroform-d) $\delta 7.44$ (dd, J = 6.7, 3.0 Hz, 2H, ArH), 7.36- $7.26(\mathrm{~m}, 3 \mathrm{H}, \mathrm{ArH}), 5.91$ (ddt, $\left.J=16.9,10.3,6.7 \mathrm{~Hz}, 1 \mathrm{H}, \mathrm{CH}=\mathrm{CH}_{2}\right), 5.32-5.18\left(\mathrm{~m}, 2 \mathrm{H}, \mathrm{CH}=\mathrm{CH}_{2}\right), 3.59\left(\mathrm{~s}, 2 \mathrm{H}, \mathrm{CH}_{2} \mathrm{C} \equiv \mathrm{C}\right), 3.18(\mathrm{~d}, J=6.6 \mathrm{~Hz}$, $\left.2 \mathrm{H}, \mathrm{CH}_{2} \mathrm{C}=\mathrm{C}\right), 2.42\left(\mathrm{~s}, 3 \mathrm{H}, \mathrm{CH}_{3}\right)$.

${ }^{13} \mathrm{C}$ NMR $(101 \mathrm{MHz}$, Chloroform-d) $\delta 134.7,131.7,128.3,128.1,123.0,118.7,85.8,83.7,59.0,45.8,41.5$. 
The tertiary amine obtained from the previous step ( $1.0 \mathrm{~g}, 3.6 \mathrm{mmol}, 1$ equiv.) was added to a solution of $\mathrm{Pd}\left(\mathrm{PPh}_{3}\right)_{4}(0.37 \mathrm{~g}, 0.32 \mathrm{~mol}, 6 \mathrm{~mol} \%)$ and 1,3-dimethylbarbituric acid (1.7 g, $11 \mathrm{mmol}, 2$ equiv.) in DCM (50 $\mathrm{mL}$ ) under an $\mathrm{N}_{2}$ atmosphere. The reaction mixture was stirred at room temperature for 6 hours. Reaction completion was checked by TLC ( $20 \%$ EtOAc in pentane). The reaction mixture was concentrated to a quarter of its original volume and diluted with ether $(40 \mathrm{~mL})$ and washed with sat. $\mathrm{NaHCO}_{3}(3 \times 15 \mathrm{~mL})$. The organic layer was extracted with aq. $\mathrm{HCl}(1.0 \mathrm{M} ; 3 \times 15 \mathrm{~mL})$ after which the combined aqueous layers and any precipitated solids were basified with $\mathrm{K}_{2} \mathrm{CO}_{3}(\mathrm{pH}>7)$ and extracted with $\mathrm{DCM}(3 \times 25 \mathrm{~mL})$. The combined extracts were dried over $\mathrm{MgSO}_{4}$, filtered and concentrated by rotary evaporation. Purification by flash column chromatography $\left(\mathrm{SiO}_{2}, 20-50 \%\right.$ EtOAc in pentane) to afford N-methyl-3-phenylprop-2-yn1 -amine ( $2 \mathrm{~h}$ ) as an orange viscous oil ( $0.46 \mathrm{~g}, 3.1 \mathrm{mmol}, 58 \%$ yield)

${ }^{1} \mathrm{H}$ NMR (400 MHz, Chloroform-d) $87.49-7.41(\mathrm{~m}, 2 \mathrm{H}, \mathrm{ArH}), 7.33(\mathrm{~m}, 3 \mathrm{H}, \mathrm{ArH}), 3.68(\mathrm{~s}, 2 \mathrm{H}, \mathrm{NCH} 2 \mathrm{C} \equiv \mathrm{C}), 3.16$ $(\mathrm{s}, 1 \mathrm{H}, \mathrm{NH}), 2.59\left(\mathrm{~s}, 3 \mathrm{H}, \mathrm{CH}_{3}\right)$.

${ }^{13} \mathrm{C}$ NMR (101 MHz, Chloroform-d) $\delta$ 131.7, 128.3, 128.1, 123.0, 86.5, 84.1, 40.5, 34.9.

Spectra data was consistent with the values reported in literature. ${ }^{2}$

\section{N-Benzyl propargylamine (7)}

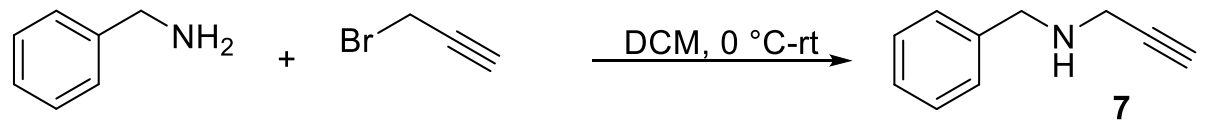

A solution of benzyl amine ( $9.8 \mathrm{~g}, 92 \mathrm{mmol}, 4$ equiv.) in $\mathrm{DCM}(15 \mathrm{~mL})$ at $0{ }^{\circ} \mathrm{C}$ was stirred vigorously while a solution of bromopropyne $(2.5 \mathrm{~mL}, 23 \mathrm{mmol}, 80 \% \mathrm{wt}$ in toluene, 1 equiv.) in DCM (10 mL) was slowly added over $10 \mathrm{~min}$. After complete addition, the reaction mixture was warmed to room temperature and stirred for 5 hours. It was then filtered through silica gel washing with 40\% EtOAc in pentane and the resulting solution concentrated. Purification was performed by column chromatography $\left(\mathrm{SiO}_{2}, 10-40 \%\right.$ EtOAc in pentane) followed by distillation $\left(70^{\circ} \mathrm{C}\right.$ at $5 \times 10^{-1} \mathrm{mbar}$ ) to obtain pure benzyl propargylamine (7) as a clear straw yellow oil $(2.2 \mathrm{~g}, 15 \mathrm{mmol}, 68 \%$ yield).

${ }^{1} \mathrm{H}$ NMR (400 MHz, Chloroform-d) $\delta 7.40-7.22(\mathrm{~m}, 5 \mathrm{H}, \mathrm{ArH}), 3.90\left(\mathrm{~s}, 2 \mathrm{H}, \mathrm{CH}_{2} \mathrm{Ar}\right.$ ), 3.44 (d, $J=2.4 \mathrm{~Hz}, 2 \mathrm{H}$, $\left.\mathrm{CH}_{2} \mathrm{C} \equiv \mathrm{C}\right), 2.27(\mathrm{t}, \mathrm{J}=2.4 \mathrm{~Hz}, 1 \mathrm{H}, \mathrm{C} \equiv \mathrm{CH}), 1.85(\mathrm{bs}, 1 \mathrm{H}, \mathrm{NH})$.

${ }^{13} \mathrm{C}$ NMR (101 MHz, Chloroform-d) $\delta$ 139.1, 128.4, 128.4, 127.2, 81.8, 71.7, 52.2, 37.3.

Spectra data was consistent with the values reported in literature. ${ }^{5}$

\section{N-Benzyl-3-(4-methoxyphenyl)prop-2-yn-1-amine (2i)}

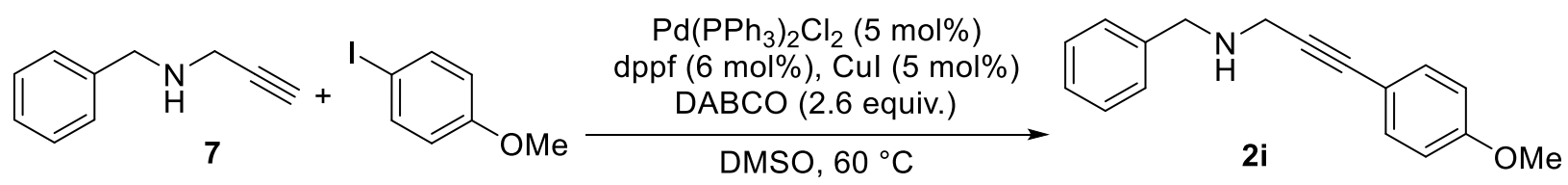

Following a modified version of a reported procedure. ${ }^{6}$ To a stirred solution of $\mathrm{Pd}\left(\mathrm{PPh}_{3}\right)_{2} \mathrm{Cl}_{2}(90 \mathrm{mg}, 0.13$ $\mathrm{mmol}, 5 \mathrm{~mol} \%$ ), dppf ( $86 \mathrm{mg}, 0.16 \mathrm{mmol}, 6 \mathrm{~mol} \%)$, Cul (25 mg, $0.13 \mathrm{mmol}, 5 \mathrm{~mol} \%)$, DABCO (0.76 g, 6.8 
mmol, 2.6 equiv.) and 4-iodo-anisole $(0.79 \mathrm{~g}, 6.4 \mathrm{mmol}, 1.3 \mathrm{mmol})$ in DMSO ( $10 \mathrm{~mL}$; degassed by bubbling $\mathrm{N}_{2}$ ) under a $\mathrm{N}_{2}$ atmosphere was added $\mathrm{N}$-Benzyl propargylamine (7) $(0.38 \mathrm{~g}, 2.6 \mathrm{mmol}, 1.0$ equiv.). The reaction mixture was heated to $60^{\circ} \mathrm{C}$ and stirred for 6 hours. The progress of the reaction was determined by TLC (30\% EtOAc in pentane). The reaction was then quenched by the addition of $\mathrm{H}_{2} \mathrm{O}(10 \mathrm{~mL})$ and the mixture diluted with EtOAc $(12 \mathrm{~mL})$. The layers were separated and the aqueous layer extracted with EtOAc $(2 \times 12 \mathrm{~mL})$. The combined organic layers were washed with brine $(2 \times 24 \mathrm{~mL})$, dried over $\mathrm{MgSO}_{4}$, which was then filtered through a pad of $\mathrm{SiO}_{2}$ (washed with EtOAc), and the filtrate was concentrated under rotary evaporation. The crude material was dry-loaded onto $\mathrm{SiO}_{2}$ and purified by column chromatography $\left(\mathrm{SiO}_{2}, 15-30 \%\right.$ EtOAc in pentane) afforded N-benzyl-3-(4-methoxyphenyl)prop-2-yn-1-amine (2i) as a light orange solid ( $0.28 \mathrm{~g}, 1.1 \mathrm{mmol}, 43 \%$ yield).

${ }^{1} \mathrm{H}$ NMR (400 MHz, Chloroform-d) $\delta 7.42-7.23(\mathrm{~m}, 7 \mathrm{H}, \mathrm{ArH}), 6.87-6.81(\mathrm{~m}, 2 \mathrm{H}, \mathrm{ArH}), 3.95(\mathrm{~s}, 2 \mathrm{H}, \operatorname{ArCH} 2)$, $3.81\left(\mathrm{~s}, 3 \mathrm{H}, \mathrm{CH}_{3}\right), 3.64\left(\mathrm{~s}, 2 \mathrm{H}, \mathrm{CH}_{2} \mathrm{C} \equiv \mathrm{C}\right), 1.64$ (bs, $\left.1 \mathrm{H}, \mathrm{NH}\right)$.

${ }^{13} \mathrm{C}$ NMR (101 MHz, Chloroform-d) $\delta 159.4,139.6,133.0,128.4$ (2C), 127.1, 115.3, 113.9, 86.0, 83.5, 55.3, $52.5,38.3$.

Spectra data was consistent with the values reported in literature. ${ }^{6}$

\section{N-Benzyl-3-(4-(trifluoromethyl)phenyl)prop-2-yn-1-amine (2j)}

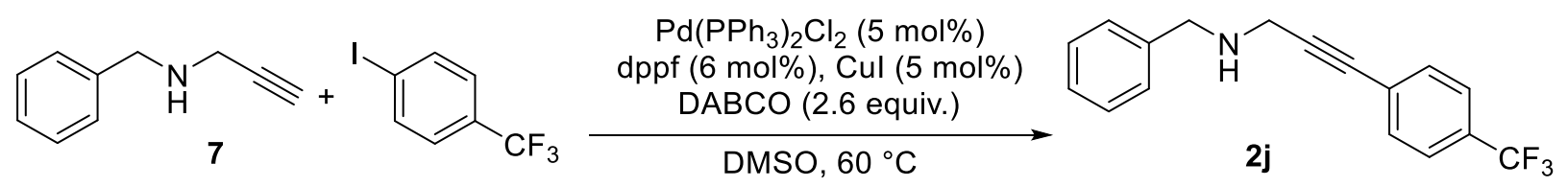

Following a modified version of a reported procedure. ${ }^{6}$ To a stirred solution of $\mathrm{Pd}\left(\mathrm{PPh}_{3}\right)_{2} \mathrm{Cl}_{2}(90 \mathrm{mg}, 0.13$ $\mathrm{mmol}, 5 \mathrm{~mol} \%$ ), dppf ( $86 \mathrm{mg}, 0.16 \mathrm{mmol}, 6 \mathrm{~mol} \%)$, Cul (25 mg, $0.13 \mathrm{mmol}, 5 \mathrm{~mol} \%)$, DABCO (0.76 g, 6.8 mmol, 2.6 equiv.) and 4-trifluro-lodobenzene $(0.92 \mathrm{~g}, 3.4 \mathrm{mmol}, 1.3$ equiv.) in DMSO (10 mL; degassed by bubbling $\mathrm{N}_{2}$ ) under a $\mathrm{N}_{2}$ atmosphere was added $\mathrm{N}$-Benzyl propargylamine (7) $(0.38 \mathrm{~g}, 2.6 \mathrm{mmol}, 1.0$ equiv.). The reaction mixture was heated to $60{ }^{\circ} \mathrm{C}$ and stirred for 6 hours. The progress of the reaction was determined by TLC ( $30 \%$ EtOAc in pentane). The reaction was then quenched by the addition of $\mathrm{H}_{2} \mathrm{O}$ (10 $\mathrm{mL})$ and the mixture diluted with EtOAc $(12 \mathrm{~mL})$. The layers were separated and the aqueous layer extracted with EtOAc $(2 \times 12 \mathrm{~mL})$. The combined organic layers were washed with brine $(2 \times 24 \mathrm{~mL})$, dried over $\mathrm{MgSO}_{4}$, which was then filtered through a pad of $\mathrm{SiO}_{2}$ (washed with $\mathrm{EtOAc}$ ), and the filtrate was concentrated under rotary evaporation. The crude material was dry-loaded onto $\mathrm{SiO}_{2}$ and purified by column chromatography $\left(\mathrm{SiO}_{2}, 10-20 \%\right.$ EtOAc in pentane) afforded N-benzyl-3-(4(trifluoromethyl)phenyl)prop-2-yn-1-amine (2j) as a dark orange oil (0.55 g, $1.9 \mathrm{mmol}, 72$ \% yield)

${ }^{1} \mathrm{H}$ NMR (400 MHz, Chloroform-d) $\left.\delta 7.61-7.24(\mathrm{~m}, 9 \mathrm{H}, \mathrm{ArH}), 3.95(\mathrm{~s}, 2 \mathrm{H}, \mathrm{ArCH})_{2}\right), 3.67\left(\mathrm{~s}, 2 \mathrm{H}, \mathrm{CH}{ }_{2} \mathrm{C} \equiv \mathrm{C}\right), 1.76$ (bs, $1 \mathrm{H}, \mathrm{NH}$ ).

${ }^{13} \mathrm{C}$ NMR (101 MHz, Chloroform-d) $\delta 139.3,131.9,129.8(q, J=32.7 \mathrm{~Hz}), 128.5,128.4,127.2,127.0,125.2$ $(q, J=3.9 \mathrm{~Hz}), 123.91(q, J=272.2 \mathrm{~Hz}), 90.2,82.5,52.6,38.2$.

${ }^{19}$ F NMR (376 MHz, Chloroform-d) $\delta$-63.2.

Spectra data was consistent with the values reported in literature. ${ }^{6}$ 


\section{N-Benzyl Propynyl trifluoroacetamide (8)}

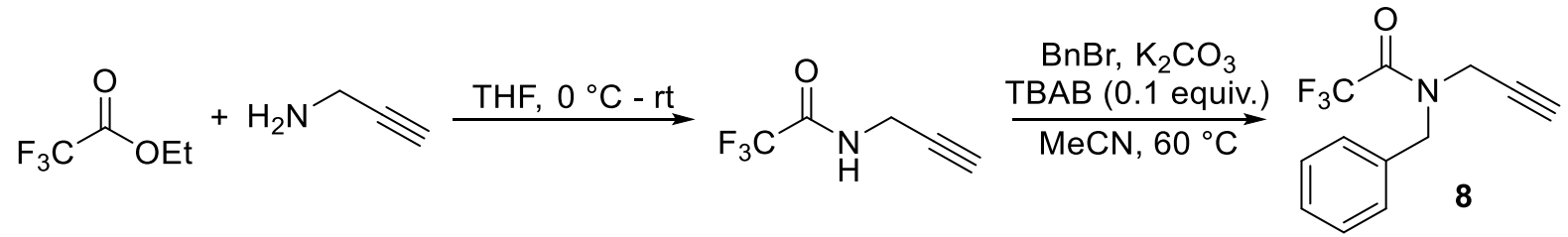

Following a modified version of a reported procedure. ${ }^{7}$ In a flame dried round bottom flask, to a solution ethyl trifluoroacetate $\left(8.0 \mathrm{~g}, 56 \mathrm{mmol}, 1.2\right.$ equiv.) in THF $(12 \mathrm{~mL})$ at $0{ }^{\circ} \mathrm{C}$ was slowly added propargylamine ( $2.6 \mathrm{~g}, 47 \mathrm{mmol}, 1$ equiv.). The reaction mixture was stirred at this temperature for 10 minutes; it was then allowed to warm to room temperature and stirred for a further 7 hours. The solvent was removed by rotary evaporation and the product was isolated by distillation $\left(90^{\circ} \mathrm{C}\right.$ at $17 \mathrm{mbar}$ ) to afford propynyl trifluoroacetamide a colourless oil (5.5 g, $37 \mathrm{mmol}, 78 \%$ yield).

${ }^{1} \mathrm{H}$ NMR (400 MHz, Chloroform-d) $\delta 6.94(\mathrm{bs}, 1 \mathrm{H}, \mathrm{NH}), 4.14$ (dd, J = 6.0, $\left.\left.2.5 \mathrm{~Hz}, 2 \mathrm{H}, \mathrm{CH}_{2} \mathrm{C} \equiv \mathrm{C},\right)^{2}\right) 2.32(\mathrm{q}, J=2.2$ $\mathrm{Hz}, 1 \mathrm{H}, \mathrm{C} \equiv \mathrm{CH})$.

${ }^{13} \mathrm{C}$ NMR (101 MHz, Chloroform-d) $\delta 157.0$ (q, $\left.J=38.1 \mathrm{~Hz}\right), 115.5$ (q, $J=287.5 \mathrm{~Hz}$ ), 77.0, 73.1, 29.6.

${ }^{19} \mathrm{~F}$ NMR (376 MHz, Chloroform-d) $\delta$-76.3.

Spectra data was consistent with the values reported in literature. ${ }^{7}$

To a mixture of $\mathrm{K}_{2} \mathrm{CO}_{3}(8.2 \mathrm{~g}, 59 \mathrm{mmol}, 2$ equiv.) and TBAB $(0.95 \mathrm{~g}, 3.0 \mathrm{mmol}, 0.1$ equiv.) in $\mathrm{MeCN}(150 \mathrm{~mL})$ was added propynyl trifluoroacetamide $(4.5 \mathrm{~g}, 30 \mathrm{mmol}, 1$ equiv.) and benzyl bromide $(6.0 \mathrm{~g}, 33 \mathrm{mmol}, 1.1$ equiv.) after which the reaction mixture was stirred at $60^{\circ} \mathrm{C}$. After 3 hours (progress determined by TLC $\left(\mathrm{SiO}_{2}, 20 \%\right.$ EtOAc in pentane)), the mixture was filtered through a plug of Celite, which was washed with $\mathrm{Et}_{2} \mathrm{O}$. The resulting filtrate was concentrated by rotary evaporation. Purification of the crude product by column chromatography $\left(\mathrm{SiO}_{2}, 0-8 \%\right.$ EtOAc in Pent) to afforded N-Benzyl propynyl trifluoroacetamide (8) as a colourless oil $(5.0 \mathrm{~g}, 21 \mathrm{mmol}, 71 \%$ yield)

${ }^{1} \mathrm{H} \mathrm{NMR}\left(400 \mathrm{MHz}\right.$, Chloroform-d; $1: 1.2$ mixture of rotamers) $\delta 7.46-7.23(\mathrm{~m}, 5 \mathrm{H}, \mathrm{ArH}), 4.79\left(\mathrm{~s}, 2 \mathrm{H}, \mathrm{CH}_{2} \mathrm{Ar}\right)$, $4.77\left(\mathrm{~s}, 2 \mathrm{H}, \mathrm{CH}_{2} \mathrm{Ar}\right), 4.12\left(\mathrm{~d}, J=2.5 \mathrm{~Hz}, 2 \mathrm{H}, \mathrm{CH}_{2} \mathrm{C} \equiv \mathrm{C}\right), 4.06\left(\mathrm{~d}, J=2.4 \mathrm{~Hz}, 2 \mathrm{H}, \mathrm{CH}_{2} \mathrm{C} \equiv \mathrm{C}\right), 2.37(\mathrm{t}, J=2.4 \mathrm{~Hz}, 1 \mathrm{H}$, $\mathrm{C} \equiv \mathrm{CH}), 2.29(\mathrm{t}, \mathrm{J}=2.5 \mathrm{~Hz}, 1 \mathrm{H}, \mathrm{C} \equiv \mathrm{CH})$.

${ }^{13} \mathrm{C}$ NMR (101 MHz, Chloroform-d; 1:1.2 mixture of rotamers) $\delta 156.7$ (q, $J=36.5 \mathrm{~Hz}$ ), 134.5, 133.8, 129.1, 129.0, 128.6, 128.6, 128.3, 127.7, 116.4 (q, $J=287.9 \mathrm{~Hz}$ ), 116.3 (q, $J=288.1 \mathrm{~Hz}$ ), 76.6 (overlapping with solvent), 76.5, 73.7, 73.3, $49.7(q, J=3.6 \mathrm{~Hz}), 48.7,35.8(q, J=4.2 \mathrm{~Hz}), 34.4$.

${ }^{19} \mathrm{~F}$ NMR (376 MHz, Chloroform- $d$; 1:1.2 mixture of rotamers) $\delta-68.5,-69.3$.

HRMS (LTQ-Orbitrap) m/z: [M + H] $]^{+}$Calcd for $\mathrm{C}_{12} \mathrm{H}_{11} \mathrm{~F}_{3} \mathrm{NO}^{+} 242.0787$; Found 242.0783. 
N-benzyl-3-(3-bromophenyl)prop-2-yn-1-amine (2k)<smiles>[C+]#CCN(Cc1ccccc1)C(=O)C(F)(F)F</smiles>

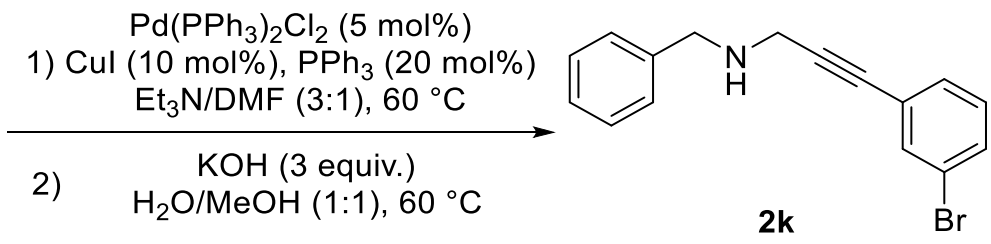

Following a modified version of a reported procedure. ${ }^{8}$ To a solution of 8 ( $0.80 \mathrm{~g}, 3.3 \mathrm{mmol}, 1$ equiv.), 1bromo-3-iodobenzene ( $0.95 \mathrm{~g}, 3.4 \mathrm{mmol}, 1.01$ equiv.) and $\mathrm{Et}_{3} \mathrm{~N}(2.3 \mathrm{~mL}, 17 \mathrm{mmol}, 5$ equiv.) in acetonitrile (30 mL) was added $\mathrm{PdCl}_{2}\left(\mathrm{PPh}_{3}\right)_{2}(47 \mathrm{mg}, 66 \mu \mathrm{mol}, 2 \mathrm{~mol} \%)$ and Cul (13 mg, $\left.66 \mu \mathrm{mol}, 2 \mathrm{~mol} \%\right)$ in a single portion. The resulting mixture was allowed to stir for 7 hours at $60^{\circ} \mathrm{C}$. Water $(20 \mathrm{~mL})$ was added and the reaction mixture extracted with EtOAc $(3 \times 30 \mathrm{~mL})$; the combined organic extracts were dried over $\mathrm{MgSO}_{4}$, filtered and concentrated by rotary evaporation. The crude material was purified by flash column chromatography $\left(\mathrm{SiO}_{2}, 0-5 \%\right.$ EtOAc in pentane) afforded N-benzyl-N-(3-(3-bromophenyl)prop-2-yn-1-yl)2,2,2-trifluoroacetamide as an yellow oil ( $1.2 \mathrm{~g}, 3.04 \mathrm{mmol}, 92 \%$ yield)

${ }^{1} \mathrm{H}$ NMR (400 MHz, Chloroform-d; 1:1.2 mixture of rotamers) $\delta 7.54(\mathrm{t}, J=1.8 \mathrm{~Hz}, 1 \mathrm{H}, \mathrm{ArH}$ ), 7.48 (dddd, $J=$ 7.9, 6.7, 2.0, $1.1 \mathrm{~Hz}, 1 \mathrm{H}, \mathrm{ArH}), 7.44-7.29(\mathrm{~m}, 6 \mathrm{H}, \mathrm{ArH}), 7.20(\mathrm{td}, J=7.9,5.4 \mathrm{~Hz}, 1 \mathrm{H}, \operatorname{ArH}), 4.83(\mathrm{~s}, 1 \mathrm{H}, \operatorname{ArCH})_{2}$, $4.81\left(\mathrm{~s}, 1 \mathrm{H}, \mathrm{ArCH}_{2}\right), 4.33\left(\mathrm{~s}, 1 \mathrm{H}, \mathrm{CH}_{2} \mathrm{C} \equiv \mathrm{C}\right), 4.30\left(\mathrm{~s}, 1 \mathrm{H}, \mathrm{CH}_{2} \mathrm{C} \equiv \mathrm{C}\right)$.

${ }^{13} \mathrm{C}$ NMR (101 MHz, Chloroform- $d ; 1: 1.2$ mixture of rotamers, some unresolved peaks) $\delta 134.7,134.6$, 134.6, 133.9, 132.1, 131.9, 130.4, 130.3, 129.8, 129.8, 129.1, 129.0, 128.6, 128.4, 127.7, 124.1, 123.8, $122.2,122.1,83.8,83.5,83.3,83.1,50.1,49.3,36.8,35.4$.

Following an adapted version of a reported procedure. ${ }^{9}$ To the trifluoroacetamide obtained from the previous step ( $0.84 \mathrm{~g}, 2.4 \mathrm{mmol}, 1$ equiv.) was added a solution of $\mathrm{KOH}(0.50 \mathrm{~g}, 9.0 \mathrm{mmol}, 3.0$ equiv.) in water $(15 \mathrm{~mL})$ and methanol $(15 \mathrm{~mL})$ and the resulting mixture was heated to $60{ }^{\circ} \mathrm{C}$ for 3 hours. The reaction was then cooled to room temperature and acidified with aq. $\mathrm{HCl}(1.0 \mathrm{M} ; 5 \mathrm{~mL})$ followed by basification with sat. aq. $\mathrm{NaHCO}_{3}(\mathrm{pH}>7)$. The resulting mixture was extracted with $\mathrm{DCM}(3 \times 10 \mathrm{~mL})$, dried over $\mathrm{MgSO}_{4}$, filtered and concentrated by rotary evaporation. Purification by column chromatography $\left(\mathrm{SiO}_{2}, 10-30 \%\right.$ EtOAc in pentane) afforded N-benzyl-3-(3-bromophenyl)prop-2-yn-1-amine (2k) as an light yellow oil $(0.80 \mathrm{~g}, 2.7 \mathrm{mmol}, 88 \%$ yield)

${ }^{1} \mathrm{H}$ NMR (400 MHz, Chloroform-d) $\delta 7.59$ (t, $\left.J=1.7 \mathrm{~Hz}, 1 \mathrm{H}, \operatorname{ArH}\right), 7.45$ (ddd, J= 8.0, 2.1, $\left.1.1 \mathrm{~Hz}, 1 \mathrm{H}, \operatorname{ArH}\right)$, $7.43-7.24(\mathrm{~m}, 6 \mathrm{H}, \mathrm{ArH}), 7.18(\mathrm{t}, J=7.9 \mathrm{~Hz}, 1 \mathrm{H}, \mathrm{ArH}), 3.96\left(\mathrm{~s}, 2 \mathrm{H}, \mathrm{ArCH}_{2}\right), 3.66\left(\mathrm{~s}, 2 \mathrm{H}, \mathrm{CH}_{2} \mathrm{C} \equiv \mathrm{C}\right), 2.37(\mathrm{~s}, 1 \mathrm{H}$, $\mathrm{NH})$.

${ }^{13} \mathrm{C}$ NMR $(101 \mathrm{MHz}$, Chloroform-d) $\delta$ 138.7, 134.4, 131.3, 130.2, 129.7, 128.5, 128.5, 127.4, 125.1, 122.1, 88.4, 82.6, 52.3, 37.9.

HRMS (ESI/QTOF) m/z: [M + H] Calcd for $\mathrm{C}_{16} \mathrm{H}_{15}{ }^{79} \mathrm{BrN}^{+}$300.0382; Found 300.0384. 
N-Benzyl-3-(3,5-dimethylphenyl)prop-2-ynylamine (2I)

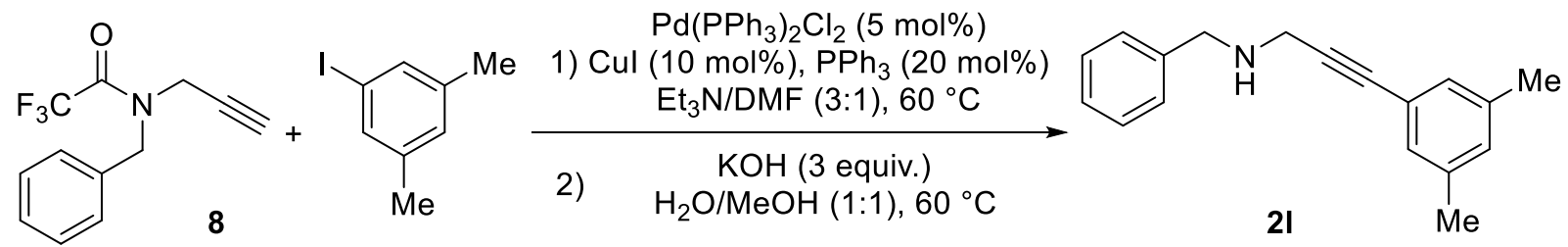

Following a modified version of a reported procedure. ${ }^{8}$ To a solution of $\mathrm{PdCl}_{2}\left(\mathrm{PPh}_{3}\right)_{2}(0.14 \mathrm{~g}, 0.20 \mathrm{mmol}, 5$ mol\%), $\mathrm{PPh}_{3}(0.21 \mathrm{~g}, 0.80 \mathrm{mmol}, 20 \mathrm{~mol} \%)$ and Cul (76 mg, $\left.0.40 \mathrm{mmol}, 10 \mathrm{~mol} \%\right)$ in DMF (3.3 mL) and Et ${ }_{3} \mathrm{~N}$ $(10 \mathrm{~mL})$ was added $7(0.97 \mathrm{~g}, 4.0 \mathrm{mmol}, 1$ equiv.) and 1-lodo-3,5-dimethylbenzene (1.1 g, $4.8 \mathrm{mmol}, 1.2$ equiv.). The resulting mixture was heated to $60^{\circ} \mathrm{C}$ for 6 hours. After cooling to room temperature, water $(25 \mathrm{~mL})$ was added and the reaction mixture extracted with EtOAc $(3 \times 40 \mathrm{~mL})$; the combined organic extracts were dried over $\mathrm{MgSO}_{4}$, filtered and concentrated by rotary evaporation. The crude material was purified by flash column chromatography $\left(\mathrm{SiO}_{2}, 0-5 \%\right.$ EtOAc in pentane) afforded N-benzyl-N-(3-(3,5dimethylphenyl)prop-2-ynyl)-trifluoroacetamide as an orange oil (1.2 g, $3.6 \mathrm{mmol}, 90 \%$ yield)

${ }^{1} \mathrm{H}$ NMR (400 MHz, Chloroform-d; mixture of rotamers) $\delta 7.44-7.29$ (m, 5, $\mathrm{CH}_{2} \mathrm{ArH}$, both rotamers), 7.06 (m, 2H, PhH, both rotamers), 7.00 (s, $1 \mathrm{H}, \mathrm{PhH}), 6.99(\mathrm{~s}, 1 \mathrm{H}, \mathrm{PhH}), 4.85$ (s, 2H, $\left.\mathrm{ArCH}_{2}\right), 4.83\left(\mathrm{~s}, 2 \mathrm{H}, \mathrm{PhCH}_{2}\right.$ ), $4.36\left(\mathrm{~s}, 2 \mathrm{H}, \mathrm{CH}_{2} \mathrm{C} \equiv \mathrm{C}\right), 4.29\left(\mathrm{~s}, 1 \mathrm{H}, \mathrm{CH}_{2} \mathrm{C} \equiv \mathrm{C}\right), 2.31\left(\mathrm{~s}, 6 \mathrm{H}, \mathrm{CH}_{3}\right), 2.30\left(\mathrm{~s}, 6 \mathrm{H}, \mathrm{CH}_{3}\right)$.

${ }^{13} \mathrm{C}$ NMR (101 MHz, Chloroform-d; mixture of rotamers, some unresolved aromatic peaks) $\delta 156.75$ (qd, J $=36.1,10.0 \mathrm{~Hz}$ ), 138.0, 138.0, 134.8, 134.1, 133.8, 133.7, 130.8, 130.7, 129.5, 129.5, 129.1, 129.0, 128.7, 128.6, 128.5, 128.5, 128.3, 127.8, 121.7, 121.4, 116.5 (qd, $J=288.1,7.9 \mathrm{~Hz}$ ), 85.8, 85.5, 81.1, 80.9, 49.74 $(q, J=3.6 \mathrm{~Hz}), 49.0,36.9(q, J=4.3 \mathrm{~Hz}), 35.4,21.1$.

HRMS (ESI/QTOF) m/z: [M + Na] ${ }^{+}$Calcd for $\mathrm{C}_{20} \mathrm{H}_{18} \mathrm{~F}_{3} \mathrm{NNaO}^{+} 368.1233$; Found 368.1235.

Following an adapted version of a reported procedure. ${ }^{9}$ To the trifluoroacetamide obtained from the previous step ( $0.84 \mathrm{~g}, 2.4 \mathrm{mmol}, 1$ equiv.) was added a solution of $\mathrm{KOH}(0.15 \mathrm{~g}, 2.7 \mathrm{mmol}, 1.3$ equiv.) in water $(5 \mathrm{~mL})$ and methanol $(5 \mathrm{~mL})$ and the resulting mixture was heated to $60{ }^{\circ} \mathrm{C}$ for 2 hours. The reaction was then cooled to room temperature and acidified with aq. $\mathrm{HCl}(1.0 \mathrm{M} ; 5 \mathrm{~mL})$ followed by basification with sat. aq. $\mathrm{NaHCO}_{3}(\mathrm{pH}>7)$. The resulting mixture was extracted with $\mathrm{DCM}(3 \times 10 \mathrm{~mL})$, dried over $\mathrm{MgSO}_{4}$, filtered and concentrated by rotary evaporation. Purification by column chromatography $\left(\mathrm{SiO}_{2}, 10-40 \%\right.$ EtOAc in pentane) afforded N-benzyl-3-(3,5-dimethylphenyl)prop-2-ynylamine (2I) as an orange oil (0.49 $\mathrm{g}, 2.0 \mathrm{mmol}, 76 \%$ yield)

${ }^{1} \mathrm{H}$ NMR $(400 \mathrm{MHz}$, Chloroform-d) $\delta 7.42-7.24(\mathrm{~m}, 5 \mathrm{H}, \operatorname{ArH}), 7.08(\mathrm{~m}, 2 \mathrm{H}, \mathrm{ArH}), 6.95(\mathrm{~m}, 1 \mathrm{H}, \operatorname{ArH}), 3.96(\mathrm{~s}$, $\left.2 \mathrm{H}, \mathrm{ArCH}_{2}\right), 3.65\left(\mathrm{~s}, 2 \mathrm{H}, \mathrm{CH}_{2} \mathrm{C} \equiv \mathrm{C}\right), 2.29\left(\mathrm{~s}, 6 \mathrm{H}, \mathrm{CH}_{3}\right), 2.09$ (bs, $\left.1 \mathrm{H}, \mathrm{NH}\right)$.

${ }^{13} \mathrm{C}$ NMR $(101 \mathrm{MHz}$, Chloroform-d) $\delta 139.3,137.8,130.0,129.3,128.5,128.4,127.2,122.8,86.5,84.2,52.3$, 38.1, 21.1.

HRMS (ESI/QTOF) m/z: [M+ H] Calcd for $\mathrm{C}_{18} \mathrm{H}_{20} \mathrm{~N}^{+}$250.1590; Found 250.1593.

Spectra data was consistent with the values reported in literature. ${ }^{4}$ 


\section{N-benzyl-3-(3-bromophenyl)prop-2-yn-1-amine (2m)}
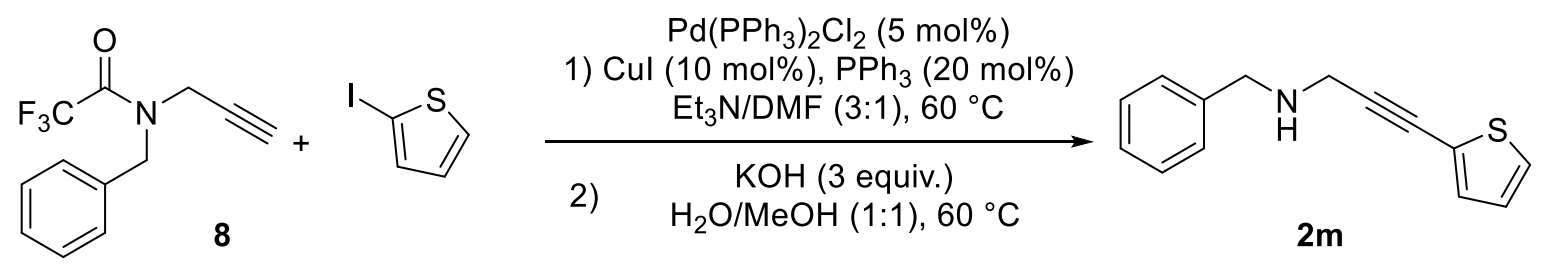

Following a modified version of a reported procedure. ${ }^{8}$ To a solution of $6(0.50 \mathrm{~g}, 2.0 \mathrm{mmol}, 1$ equiv. $)$ and 2-iodothiophene ( $0.43 \mathrm{~g}, 2.0 \mathrm{mmol}, 1.01$ equiv.) and $\mathrm{Et}_{3} \mathrm{~N}(1.4 \mathrm{~mL}, 10 \mathrm{mmol}, 5$ equiv.) in acetonitrile (30 $\mathrm{mL}$ ) was added $\mathrm{PdCl}_{2}\left(\mathrm{PPh}_{3}\right)_{2}(36 \mathrm{mg}, 51 \mu \mathrm{mol}, 2 \mathrm{~mol} \%)$ and $\mathrm{Cul}(12 \mathrm{mg}, 66 \mu \mathrm{mol}, 3 \mathrm{~mol} \%)$ in a single portion. The resulting mixture was stirred at room temperature for 7 hours. Water $(20 \mathrm{~mL})$ was added and the reaction mixture extracted with EtOAc $(3 \times 30 \mathrm{~mL})$; the combined organics portions were dried over $\mathrm{MgSO}_{4}$, filtered and concentrated by rotary evaporation. The crude material was purified by flash column chromatography $\left(\mathrm{SiO}_{2}, 0-5 \%\right.$ EtOAc in pentane) afforded $\mathrm{N}$-benzyl-2,2,2-trifluoro- $\mathrm{N}$-(3-(thiophen-2yl)prop-2-yn-1-yl)acetamide as an yellow oil $(0.58 \mathrm{~g}, 1.8 \mathrm{mmol}, 88 \%$ yield).

${ }^{1} \mathrm{H}$ NMR (400 MHz, Chloroform-d mixture of rotamers) $\delta 7.44-7.27(\mathrm{~m}, 6 \mathrm{H}, \mathrm{ArH}), 7.22$ (ddd, J = 4.9, 3.6, $1.2 \mathrm{~Hz}, 1 \mathrm{H}, \mathrm{ArH}), 6.99(\mathrm{td}, J=5.2,3.6 \mathrm{~Hz}, 1 \mathrm{H}, \mathrm{ArH}), 4.82\left(\mathrm{~d}, J=8.8 \mathrm{~Hz}, 2 \mathrm{H}, \mathrm{ArCH}_{2}\right), 4.35(\mathrm{~d}, J=23.4 \mathrm{~Hz}, 2 \mathrm{H}$, $\left.\mathrm{CH}_{2} \mathrm{C} \equiv \mathrm{C}\right)$.

${ }^{13} \mathrm{C}$ NMR (101 MHz, Chloroform- $d$ mixture of rotamers, some unresolved peaks) $\delta 134.7,134.0,133.0$, $132.8,129.1$, 129.0, 128.6, 128.5, 128.3, 127.9, 127.8, 127.7, 127.1, 127.0, 122.0, 121.6, 85.9, 85.6, 78.7, $78.5,50.0,49.2,37.0,35.6$.

Following an adapted version of a reported procedure. ${ }^{9}$ To the trifluoroacetamide obtained from the previous step ( $0.58 \mathrm{~g}, 1.8 \mathrm{mmol}, 1$ equiv.) was added a solution of $\mathrm{KOH}(0.30 \mathrm{~g}, 5.4 \mathrm{mmol}, 3.0$ equiv.) in water $(9 \mathrm{~mL})$ and methanol $(9 \mathrm{~mL})$ and the resulting mixture was heated to $60^{\circ} \mathrm{C}$ for 4 hours. The reaction was then cooled to room temperature and acidified with aq. $\mathrm{HCl}(1.0 \mathrm{M} ; 5 \mathrm{~mL})$ followed by basification with sat. aq. $\mathrm{NaHCO}_{3}(\mathrm{pH}>7)$. The resulting mixture was extracted with $\mathrm{DCM}(3 \times 10 \mathrm{~mL})$, dried over $\mathrm{MgSO}_{4}$, filtered and concentrated by rotary evaporation. Purification by column chromatography $\left(\mathrm{SiO}_{2}, 10-30 \%\right.$ EtOAc in pentane) afforded N-benzyl-3-(3-bromophenyl)prop-2-yn-1-amine ( $\mathbf{2 m}$ ) as an orange amorphous solid (0.38 g, $1.7 \mathrm{mmol}, 93 \%$ yield)

${ }^{1} \mathrm{H}$ NMR (400 MHz, Chloroform-d) $87.40-7.27$ (m, 5H, ArH), 7.24 (dd, J = 5.2, 1.2 Hz, 1H, ArH), 7.20 (dd, J $=3.6,1.1 \mathrm{~Hz}, 1 \mathrm{H}, \mathrm{ArH}), 6.97(\mathrm{dd}, J=5.2,3.6 \mathrm{~Hz}, 1 \mathrm{H}, \operatorname{ArH}), 3.95\left(\mathrm{~s}, 2 \mathrm{H}, \mathrm{ArCH}_{2}\right), 3.68\left(\mathrm{~s}, 2 \mathrm{H}, \mathrm{CH}_{2} \mathrm{C} \equiv \mathrm{C}\right), 3.00(\mathrm{~s}$, $1 \mathrm{H} \mathrm{NH).}$

${ }^{13} \mathrm{C}$ NMR (101 MHz, Chloroform-d) $\delta 138.8,131.8,128.5,128.5,127.3,126.9,126.8,123.1,91.0,77.3,52.3$, 38.2 .

HRMS (ESI/QTOF) m/z: [M + H] Calcd for $\mathrm{C}_{14} \mathrm{H}_{14} \mathrm{NS}^{+} 228.0841$; Found 228.0844. 


\section{Synthesis of Bromoalkyne}

triisopropylsilyl bromoacetylene (1)

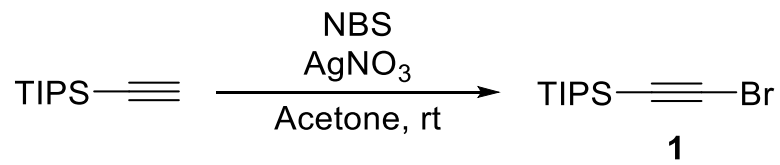

Following a reported procedure.$^{10}$ triisopropylsilylacetylene $(0.81 \mathrm{~g}, 4.5 \mathrm{mmol}, 1.00$ equiv.) was dissolved in acetone $(30 \mathrm{~mL})$. $\mathrm{N}$-bromosuccinimide $\left(0.93 \mathrm{~g}, 5.2 \mathrm{mmol}, 1.16\right.$ equiv.) was added, followed by $\mathrm{AgNO}_{3}$ ( $76 \mathrm{mg}, 0.44 \mathrm{mmol}, 0.1$ equiv.). The resulting mixture was stirred at room temperature for $3 \mathrm{~h}$ and it was then poured onto ice. After ice being allowed to melt, the aqueous layer was extracted with pentane $(3 \mathrm{x}$ $30 \mathrm{~mL}$ ). The combined organic layers were dried over $\mathrm{MgSO}_{4}$, filtered through a plug of silica and concentrated by rotary evaporation to afford pure triisopropylsilyl bromoacetylene (1) as a colourless oil. (1.16 g, $4.43 \mathrm{mmol}, 99 \%$ yield)

${ }^{1} \mathrm{H}$ NMR (400 MHz, Chloroform-d) $\delta 1.20-0.97$ (m, 21H, TIPS).

${ }^{13} \mathrm{C}$ NMR (100 MHz, Chloroform-d) $\delta$ 83.5, 61.7, 18.5, 11.3.

Spectra data was consistent with the values reported in literature. ${ }^{10}$

Procedure copied from previous publication from the group. ${ }^{11}$

\section{Optimisation of the Carbo-Oxygenation reaction.}

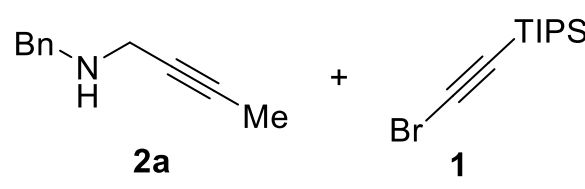

$0.1-0.2 \mathrm{mmol}$

1.3 equiv.

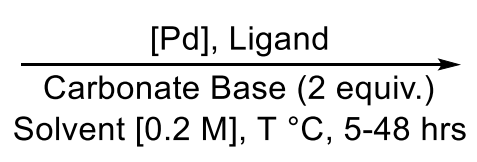
Solvent $[0.2 \mathrm{M}], \mathrm{T}^{\circ} \mathrm{C}, 5-48 \mathrm{hrs}$<smiles></smiles>

\begin{tabular}{|c|c|c|c|c|c|c|c|}
\hline Entry & Pd source & Ligand & Base & Solvent & $\mathrm{T}\left({ }^{\circ} \mathrm{C}\right)$ & Yield $^{a}$ & SM \\
\hline 1 & $\mathrm{Pd}_{2}(\mathrm{dba})_{3} . \mathrm{CHCl}_{3}(2.5 \mathrm{~mol} \%)$ & DPEPhos & $\mathrm{Cs}_{2} \mathrm{CO}_{3}$ & DCE & 60 & 44 & 14 \\
\hline $2^{[c]}$ & $\mathrm{Pd}_{2}(\mathrm{dba})_{3} . \mathrm{CHCl}_{3}(2.5 \mathrm{~mol} \%)$ & DPEPhos & $\mathrm{Cs}_{2} \mathrm{CO}_{3}$ & DCE & 60 & 80 & 0 \\
\hline 3 & $\mathrm{Pd}_{2}(\mathrm{dba})_{3} . \mathrm{CHCl}_{3}(2.5 \mathrm{~mol} \%)$ & DPEPhos & $\mathrm{Cs}_{2} \mathrm{CO}_{3}$ & DCE & 75 & 40 & 6 \\
\hline 4 & $\mathrm{Pd}_{2}(\mathrm{dba})_{3} . \mathrm{CHCl}_{3}(2.5 \mathrm{~mol} \%)$ & DPEPhos & $\mathrm{Li}_{2} \mathrm{CO}_{3}$ & DCE & 75 & 0 & 36 \\
\hline 5 & $\mathrm{Pd}_{2}(\mathrm{dba})_{3} . \mathrm{CHCl}_{3}(2.5 \mathrm{~mol} \%)$ & DPEPhos & $\mathrm{Na}_{2} \mathrm{CO}_{3}$ & DCE & 75 & 13 & 31 \\
\hline 6 & $\mathrm{Pd}_{2}(\mathrm{dba})_{3} . \mathrm{CHCl}_{3}(2.5 \mathrm{~mol} \%)$ & DPEPhos & $\mathrm{K}_{2} \mathrm{CO}_{3}$ & DCE & 75 & 12 & \\
\hline 7 & $\mathrm{Pd}_{2}(\mathrm{dba})_{3} . \mathrm{CHCl}_{3}(2.5 \mathrm{~mol} \%)$ & DPEPhos & $\mathrm{Rb}_{2} \mathrm{CO}_{3}$ & DCE & 75 & 40 & - \\
\hline 8 & $\mathrm{Pd}_{2}(\mathrm{dba})_{3} . \mathrm{CHCl}_{3}(2.5 \mathrm{~mol} \%)$ & DPEPhos & $\mathrm{BaCO}_{3}$ & DCE & 75 & 0 & - \\
\hline 9 & $\mathrm{Pd}_{2}(\mathrm{dba})_{3} . \mathrm{CHCl}_{3}(2.5 \mathrm{~mol} \%)$ & DPEPhos & $\mathrm{CaCO}_{3}$ & DCE & 75 & 0 & - \\
\hline 10 & $\mathrm{Pd}_{2}(\mathrm{dba})_{3} . \mathrm{CHCl}_{3}(2.5 \mathrm{~mol} \%)$ & DPEPhos & $\mathrm{NaHCO}_{3}$ & DCE & 75 & 14 & - \\
\hline 11 & $\mathrm{Pd}_{2}(\mathrm{dba})_{3} . \mathrm{CHCl}_{3}(2.5 \mathrm{~mol} \%)$ & DPEPhos & $\mathrm{CsHCO}_{3}$ & DCE & 75 & 64 & - \\
\hline 12 & $\mathrm{Pd}_{2}(\mathrm{dba})_{3} . \mathrm{CHCl}_{3}(2.5 \mathrm{~mol} \%)$ & DPEPhos & $\mathrm{CsHCO}_{3}$ & DCE & 60 & 73 & - \\
\hline
\end{tabular}




\begin{tabular}{|c|c|c|c|c|c|c|c|}
\hline 13 & $\mathrm{Pd}_{2}(\mathrm{dba})_{3} . \mathrm{CHCl}_{3}(2.5 \mathrm{~mol} \%)$ & DCEPhos & $\mathrm{CsHCO}_{3}$ & DCE & 60 & 2 & 15 \\
\hline 14 & $\mathrm{Pd}_{2}(\mathrm{dba})_{3} \cdot \mathrm{CHCl}_{3}(2.5 \mathrm{~mol} \%)$ & XantPhos & $\mathrm{CsHCO}_{3}$ & DCE & 60 & 4 & 50 \\
\hline 15 & $\mathrm{Pd}_{2}(\mathrm{dba})_{3} . \mathrm{CHCl}_{3}(2.5 \mathrm{~mol} \%)$ & dppf & $\mathrm{CsHCO}_{3}$ & DCE & 60 & 50 & 20 \\
\hline 16 & $\mathrm{Pd}_{2}(\mathrm{dba})_{3} . \mathrm{CHCl}_{3}(2.5 \mathrm{~mol} \%)$ & dppe & $\mathrm{CsHCO}_{3}$ & DCE & 60 & 8 & 19 \\
\hline 17 & $\mathrm{Pd}_{2}(\mathrm{dba})_{3 .} \mathrm{CHCl}_{3}(2.5 \mathrm{~mol} \%)$ & RuPhos & $\mathrm{CsHCO}_{3}$ & DCE & 60 & 3 & 46 \\
\hline 18 & $\mathrm{Pd}_{2}(\mathrm{dba})_{3} . \mathrm{CHCl}_{3}(2.5 \mathrm{~mol} \%)$ & DPEPhos & $\mathrm{CsHCO}_{3}$ & Cl-Benzene & 60 & 21 & 22 \\
\hline 19 & $\mathrm{Pd}_{2}(\mathrm{dba})_{3} . \mathrm{CHCl}_{3}(2.5 \mathrm{~mol} \%)$ & DPEPhos & $\mathrm{CsHCO}_{3}$ & CPME & 60 & 7 & 31 \\
\hline 20 & $\mathrm{Pd}_{2}(\mathrm{dba})_{3 .} \mathrm{CHCl}_{3}(2.5 \mathrm{~mol} \%)$ & DPEPhos & $\mathrm{CsHCO}_{3}$ & EtOAc & 60 & 15 & 27 \\
\hline 21 & $\mathrm{Pd}_{2}(\mathrm{dba})_{3} . \mathrm{CHCl}_{3}(2.5 \mathrm{~mol} \%)$ & DPEPhos & $\mathrm{CsHCO}_{3}$ & $\mathrm{~F}_{3}$ Toluene & 60 & 41 & 5 \\
\hline 22 & $\mathrm{Pd}_{2}(\mathrm{dba})_{3 .} \mathrm{CHCl}_{3}(2.5 \mathrm{~mol} \%)$ & DPEPhos & $\mathrm{CsHCO}_{3}$ & DCM & 60 & 50 & 0 \\
\hline 23 & $\mathrm{Pd}_{2}(\mathrm{dba})_{3 .} \mathrm{CHCl}_{3}(2.5 \mathrm{~mol} \%)$ & DPEPhos & $\mathrm{CsHCO}_{3}$ & $\mathrm{CHCl}_{3}$ & 60 & 4 & 4 \\
\hline 24 & $\mathrm{Pd}(\mathrm{OAc})_{2 .} .(5 \mathrm{~mol} \%)$ & DPEPhos & $\mathrm{CsHCO}_{3}$ & DCE & 60 & 15 & 5 \\
\hline 25 & $\mathrm{Pd}(\mathrm{acac}) 2 .(5 \mathrm{~mol} \%)$ & DPEPhos & $\mathrm{CsHCO}_{3}$ & DCE & 60 & 37 & 2 \\
\hline 26 & $\mathrm{Pd}\left(\mathrm{PPh}_{3}\right)_{2} \mathrm{Cl}_{2 .} .(5 \mathrm{~mol} \%)$ & DPEPhos & $\mathrm{CsHCO}_{3}$ & DCE & 60 & 3 & 3 \\
\hline 27 & $\mathrm{Pd}(\mathrm{dba})_{2 .} .(5 \mathrm{~mol} \%)$ & DPEPhos & $\mathrm{CsHCO}_{3}$ & DCE & 60 & 48 & 50 \\
\hline 28 & $\mathrm{Pd}$ (DPEPhos) $\mathrm{Cl}_{2 .}(5 \mathrm{~mol} \%)$ & DPEPhos & $\mathrm{CsHCO}_{3}$ & DCE & 60 & 14 & 35 \\
\hline 29 & $\mathrm{Pd}_{2}(\mathrm{dba})_{3} . \mathrm{CHCl}_{3}(2.5 \mathrm{~mol} \%)$ & DPEPhos & $\mathrm{CsHCO}_{3}$ & DCE & 50 & 64 & 7 \\
\hline 30 & $\mathrm{Pd}_{2}(\mathrm{dba})_{3} . \mathrm{CHCl}_{3}(1.25 \mathrm{~mol} \%)$ & DPEPhos & $\mathrm{CsHCO}_{3}$ & DCE & 60 & 68 & 5 \\
\hline 31 & $\mathrm{Pd}_{2}(\mathrm{dba})_{3} . \mathrm{CHCl}_{3}(2.5 \mathrm{~mol} \%)$ & DPEPhos & $\mathrm{CsHCO}_{3}{ }^{[\mathrm{b}]}$ & DCE & 60 & 69 & 5 \\
\hline $32^{[c]}$ & $\mathrm{Pd}_{2}\left(\mathrm{dba}_{3} . \mathrm{CHCl}_{3}(2.5 \mathrm{~mol} \%)\right.$ & DPEPhos & $\mathrm{CsHCO}_{3}$ & DCE & 60 & 80 & 0 \\
\hline $33^{[d]}$ & $\mathrm{Pd}_{2}(\mathrm{dba})_{3 .} \mathrm{CHCl}_{3}(2.5 \mathrm{~mol} \%)$ & DPEPhos & $\mathrm{CsHCO}_{3}$ & DCE & 60 & 50 & 30 \\
\hline $34^{[\mathrm{e}]}$ & $\mathrm{Pd}_{2}(\mathrm{dba})_{3 .} \mathrm{CHCl}_{3}(2.5 \mathrm{~mol} \%)$ & DPEPhos & $\mathrm{CsHCO}_{3}$ & DCE & 60 & $80(77)$ & 0 \\
\hline 35 & $\mathrm{Pd}(\mathrm{Cp})$ cinnamyl (5 mol\%) & DPEPhos & $\mathrm{CsHCO}_{3}$ & DCE & 60 & 73 & 0 \\
\hline $34^{[f]}$ & $\mathrm{Pd}_{2}(\mathrm{dba})_{3 .} \mathrm{CHCl}_{3}(2.5 \mathrm{~mol} \%)$ & DPEPhos & $\mathrm{CsHCO}_{3}$ & DCE & 60 & 76 & 0 \\
\hline
\end{tabular}

[a] Yields were calculated from ${ }^{1} \mathrm{H}$ NMR spectra by using 1,2-diflurobenzene as internal standard. [b] 1 equiv Base. [c] $\mathrm{CO}_{2}$ balloon. [d] $0.4 \mathrm{mmol}$ scale, inneficient stirring. [e] $0.4 \mathrm{mmol}$ scale, effective stirring. [f] addition of 4 ÅMs.

\section{Scope of the Carbo-Oxygenation Reaction.}

\section{General procedure for the carboxylation-alkynylation reaction of propargylic amines}

A vial was charged with $\mathrm{Pd}_{2}(\mathrm{dba})_{3} \cdot \mathrm{CHCl}_{3}(10.4 \mathrm{mg}, 10.0 \mu \mathrm{mol}, 2.5 \mathrm{~mol} \%)$, DPEPhos (16.0 mg, $30.0 \mu \mathrm{mol}, 7.5$ mol\%) and $\mathrm{CsHCO}_{3}(155 \mathrm{mg}, 0.520 \mathrm{mmol}, 2.0$ equiv.). The vial was sealed and purged with argon. To this was added DCE $(2.0 \mathrm{~mL})$ and the mixture was heated at $60{ }^{\circ} \mathrm{C}$ for $2-3$ minutes, after which it took on an orange colour. Internal propargylic amine $2 \mathrm{a}-\mathrm{m}(0.400 \mathrm{mmol})$, and triisopropylsilyl bromoacetylene 1 (136 $\mathrm{mg}, 0.520 \mathrm{mmol}, 1.3$ equiv.) were then added directly to the reaction mixture and left to stir at $60^{\circ} \mathrm{C}$ for 18-24 hours. After this time, the reaction mixture was removed from heating, cooled to room temperature, unsealed and diluted with pentane $(4 \mathrm{~mL})$ and passed through a small plug of silica gel (deactivated by $\mathrm{Et}_{3} \mathrm{~N}$ ) rinsing with $10 \%$ EtOAc in pentane $(8 \mathrm{~mL})$ and concentrated by rotary evaporation. The crude material was dry-loaded onto deactivated silica gel prior to column chromatography.

\section{(E)-3-benzyl-5-(4-(triisopropylsilyl)but-3-yn-2-ylidene)oxazolidin-2-one (5a)}




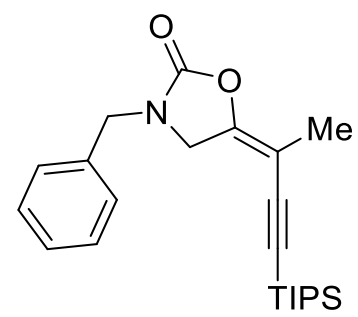

Using $2 \mathrm{a}(58.1 \mathrm{mg}, 0.400 \mathrm{mmol})$. The crude product was purified by column chromatography $\left(\mathrm{SiO}_{2} ; 0-3 \%\right.$ EtOAc in pentane) afforded (E)-3-benzyl-5-(4-(triisopropylsilyl)but-3-yn-2-ylidene)oxazolidin-2-one (5a) as a straw yellow oil (119 $\mathrm{mg}, 0.309 \mathrm{mmol}, 77 \%$ yield)

${ }^{1} \mathrm{H}$ NMR (400 MHz, Chloroform-d) $\delta 7.47-7.24(\mathrm{~m}, 5 \mathrm{H}, \mathrm{ArH}), 4.51\left(\mathrm{~s}, 2 \mathrm{H}, \mathrm{ArCH}_{2}\right), 4.12(\mathrm{q}, J=2.2 \mathrm{~Hz}, 2 \mathrm{H}$, $\left.\mathrm{NCH}_{2} \mathrm{C}=\mathrm{C}\right), 1.84\left(\mathrm{t}, \mathrm{J}=2.2 \mathrm{~Hz}, 3 \mathrm{H}, \mathrm{CH}_{3}\right), 1.06-1.00(\mathrm{~m}, 21 \mathrm{H}, \mathrm{TIPS})$.

${ }^{13} \mathrm{C}$ NMR (101 MHz, Chloroform-d) $\delta$ 155.1, 148.7, 134.7, 129.0, 128.3 (2C), 104.0, 94.6, 94.4, 48.0, 48.0, 18.6, 14.7, 11.1 .

IR $\left(v_{\max }, \mathrm{cm}^{-1}\right) 2942(\mathrm{~m}), 2865(\mathrm{~m}), 2141(\mathrm{w}), 1794(\mathrm{~s}), 1686(\mathrm{~s}), 1469(\mathrm{~m}), 1418(\mathrm{~m}), 1244(\mathrm{~m}), 1081(\mathrm{~m})$, $1047(s)$

HRMS (ESI/QTOF) m/z: [M + Na] ${ }^{+}$Calcd for $\mathrm{C}_{23} \mathrm{H}_{33} \mathrm{NNaO}_{2} \mathrm{Si}^{+} 406.2173$; Found 406.2168.

\section{(E)-3-(4-methoxybenzyl)-5-(4-(triisopropylsilyl)but-3-yn-2-ylidene)oxazolidin-2-one (5b)}<smiles></smiles>

Using $\mathbf{2 b}$ (76.0 $\mathrm{mg}, 0.400 \mathrm{mmol})$. The crude product was purified by column chromatography $\left(\mathrm{SiO}_{2} ; 0-3 \%\right.$ EtOAc in pentane) afforded (E)-3-(4-methoxybenzyl)-5-(4-(triisopropylsilyl)but-3-yn-2-ylidene)oxazolidin2-one (5b) as a straw yellow oil (127 mg, $0.293 \mathrm{mmol}, 77 \%$ yield)

${ }^{1} \mathrm{H}$ NMR (400 MHz, Chloroform-d) $\left.\delta 7.20(\mathrm{~m}, 2 \mathrm{H}, \mathrm{ArH}), 6.89(\mathrm{~m}, 2 \mathrm{H}, \mathrm{ArH}), 4.42(\mathrm{~s}, 2 \mathrm{H}, \operatorname{ArCH})^{2}\right), 4.07(\mathrm{q}, J=$ $\left.2.2 \mathrm{~Hz}, 2 \mathrm{H}, \mathrm{NCH}_{2} \mathrm{C}=\mathrm{C}\right), 3.81\left(\mathrm{~s}, 3 \mathrm{H}, \mathrm{OCH}_{3}\right), 1.81\left(\mathrm{t}, \mathrm{J}=2.2 \mathrm{~Hz}, 3 \mathrm{H}, \mathrm{CH}_{3}\right), 1.05-1.00(\mathrm{~m}, 21 \mathrm{H}, \mathrm{TIPS})$.

${ }^{13}$ C NMR (101 MHz, Chloroform-d) $\delta$ 159.6, 155.0, 148.9, 129.7, 126.8, 114.3, 104.1, 94.5, 94.3, 55.3, 47.9, 47.3, 18.6, 14.6, 11.1 .

IR ( $\left.v_{\max }, \mathrm{cm}^{-1}\right) 2946(\mathrm{~m}), 2868(\mathrm{~m}), 2140(\mathrm{w}), 1789(\mathrm{~s}), 1686(\mathrm{~s}), 1515(\mathrm{~s}), 1468(\mathrm{~m}), 1416(\mathrm{~m}), 1248(\mathrm{~s}), 1042$ (s), $679(\mathrm{~s}), 661(\mathrm{~s})$

HRMS (ESI/QTOF) m/z: [M + Na] ${ }^{+}$Calcd for $\mathrm{C}_{24} \mathrm{H}_{35} \mathrm{NNaO}_{3} \mathrm{Si}^{+} 436.2278$; Found 436.2291.

\section{(E)-3-ferrocenylmethyl-5-(4-(triisopropylsilyl)but-3-yn-2-ylidene)oxazolidin-2-one (5c)}




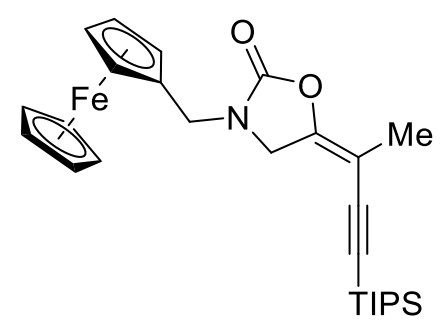

Using $2 \mathrm{c}$ (107 $\mathrm{mg}, 0.400 \mathrm{mmol})$. The crude product was purified by column chromatography $\left(\mathrm{SiO}_{2} ; 0-3 \%\right.$ EtOAc in pentane) afforded (E)-3-ferrocenylmethyl-5-(4-(triisopropylsilyl)but-3-yn-2-ylidene)oxazolidin-2one (5c) as an amorphous orange solid (127 $\mathrm{mg}, 0.260 \mathrm{mmol}, 65 \%$ yield) the product was isolated with $6.5 \%$ dibenzylideneacetone which was removed upon preparative TLC (10\% $\mathrm{Et}_{2} \mathrm{O}$ in pentane).

${ }^{1} \mathrm{H}$ NMR $\left(400 \mathrm{MHz}\right.$, Chloroform-d) $\delta 4.28\left(\mathrm{~s}, 2 \mathrm{H}\right.$, ferroceneCH $\left.\mathrm{C}_{2}\right), 4.17(\mathrm{~d}, J=3.7 \mathrm{~Hz}, 9 \mathrm{H}$ ferroceneH), 4.11 (q, $\left.J=2.2 \mathrm{~Hz}, 2 \mathrm{H}, \mathrm{NCH}_{2} \mathrm{C}=\mathrm{C}\right), 1.78\left(\mathrm{t}, J=2.1 \mathrm{~Hz}, 3 \mathrm{H}, \mathrm{CH}_{3}\right), 1.04(\mathrm{~m}, J=1.6 \mathrm{~Hz}, 21 \mathrm{H}, \mathrm{TIPS})$.

${ }^{13} \mathrm{C}$ NMR (101 MHz, Chloroform-d) $\delta$ 154.6, 149.0, 104.2, 94.3, 94.1, 80.6, 69.0, 68.8, 68.7, 48.0, 43.5, 18.6, $14.5,11.1$.

IR ( $\left.v_{\max }, \mathrm{cm}^{-1}\right) 2943(\mathrm{~m}), 2864(\mathrm{~m}), 2146(\mathrm{w}), 1788(\mathrm{~s}), 1772(\mathrm{~s}), 1688(\mathrm{~s}), 1468(\mathrm{~m}), 1313(\mathrm{~m}), 1245(\mathrm{~s}), 1198$ $(\mathrm{m}), 1058(\mathrm{~s}), 665(\mathrm{~s})$

HRMS (ESI/QTOF) m/z: [M] ${ }^{+}$Calcd for $\mathrm{C}_{27} \mathrm{H}_{37} \mathrm{FeNO}_{2} \mathrm{Si}^{+}$491.1937; Found 491.1939.

\section{(E)-3-benzyl-5-(1-phenyl-4-(triisopropylsilyl)but-3-yn-2-ylidene)oxazolidin-2-one (5d)}

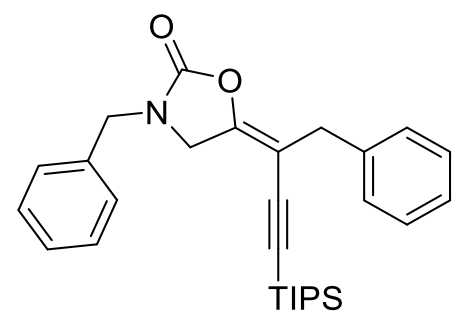

Using $2 \mathbf{d}(94.0 \mathrm{mg}, 0.400 \mathrm{mmol})$. The crude product was purified by column chromatography (Et $3 \mathrm{~N}-$ deactivated $\mathrm{SiO}_{2} ; 0-2 \%$ EtOAc in pentane) afforded (E)-3-benzyl-5-(1-phenyl-4-(triisopropylsilyl)but-3-yn2-ylidene)oxazolidin-2-one (5d) as an orange oil (110 mg, $0.239 \mathrm{mmol}, 60 \%$ yield) [10\% SM in crude NMR] ${ }^{1} \mathrm{H}$ NMR (400 MHz, Chloroform-d) $\delta 7.42-7.14(\mathrm{~m}, 10 \mathrm{H}, \operatorname{ArH}), 4.49\left(\mathrm{~s}, 2 \mathrm{H}, \mathrm{ArCH}_{2} \mathrm{~N}\right), 4.12(\mathrm{t}, J=1.5 \mathrm{~Hz}, 2 \mathrm{H}$, $\left.\mathrm{NCH}_{2} \mathrm{C}=\mathrm{C}\right), 3.51\left(\mathrm{~d}, \mathrm{~J}=1.5 \mathrm{~Hz}, 2 \mathrm{H}, \mathrm{ArCH}_{2} \mathrm{C}\right), 0.99-0.92(\mathrm{~m}, 21 \mathrm{H}, \mathrm{TIPS})$.

${ }^{13} \mathrm{C}$ NMR (101 MHz, Chloroform-d) $\delta 154.9,148.7,138.5,134.6,129.0,128.9,128.4$ (2C), 128.3, 126.4, $102.8,99.1,96.6,48.1,48.0,34.8,18.5,11.1$.

IR $\left(v_{\max }, \mathrm{cm}^{-1}\right) 882(\mathrm{~s}), 1045(\mathrm{~s}), 1248(\mathrm{~s}), 1417(\mathrm{~s}), 1469(\mathrm{~s}), 1672(\mathrm{~s}), 1794(\mathrm{~s}), 2141(\mathrm{~s}), 2864(\mathrm{~s}), 2941(\mathrm{~s})$ HRMS (ESI/QTOF) m/z: [M + Na] ${ }^{+}$Calcd for $\mathrm{C}_{29} \mathrm{H}_{37} \mathrm{NNaO}_{2} \mathrm{Si}^{+} 482.2486$; Found 482.2495.

\section{(E)-3-benzyl-5-(1-cyclopropyl-3-(triisopropylsilyl)prop-2-yn-1-ylidene)oxazolidin-2-one (5e)}




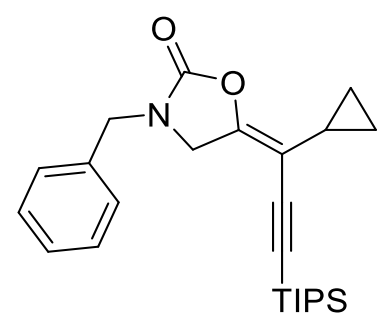

Using $2 \mathrm{e}(74.0 \mathrm{mg}, 0.400 \mathrm{mmol})$. The crude product was purified by column chromatography $\left(\mathrm{SiO}_{2} ; 0-2 \%\right.$ EtOAc in pentane) afforded (E)-3-benzyl-5-(1-cyclopropyl-3-(triisopropylsilyl)prop-2-yn-1-ylidene) oxazolidin-2-one (5e) as a dark orange oil (135 $\mathrm{mg}, 0.329 \mathrm{mmol}, 82 \%$ yield)

${ }^{1} \mathrm{H}$ NMR (400 MHz, Chloroform-d) $\delta 7.41-7.23(\mathrm{~m}, 5 \mathrm{H}, \mathrm{ArH}), 4.49\left(\mathrm{~s}, 2 \mathrm{H}, \mathrm{ArCH}_{2}\right), 4.12\left(\mathrm{~s}, 2 \mathrm{H}, \mathrm{NCH} \mathrm{C}_{2} \mathrm{C}\right)$, $1.81\left(\mathrm{tt}, J=8.0,5.2 \mathrm{~Hz}, 1 \mathrm{H}, \mathrm{CH}\left(-\mathrm{CH}_{2} \mathrm{CH}_{2}-\right)\right), 1.08-0.95(\mathrm{~m}, 21 \mathrm{H}, \mathrm{TIPS}), 0.76-0.60\left(\mathrm{~m}, 4 \mathrm{H}, \mathrm{CH}\left(-\mathrm{CH}_{2} \mathrm{CH}_{2}-\right)\right)$.

${ }^{13} \mathrm{C}$ NMR (101 MHz, Chloroform-d) $\delta$ 155.1, 148.0, 134.7, 128.9, 128.2 (2C), 102.0, 99.3, 96.5, 48.3, 47.9, $18.5,11.0,8.3,4.8$.

IR $\left(v_{\max }, \mathrm{cm}^{-1}\right) 2945(\mathrm{~m}), 2865(\mathrm{~m}), 2150(\mathrm{w}), 1791(\mathrm{~s}), 1671(\mathrm{~s}), 1467(\mathrm{~m}), 1419(\mathrm{~m}), 1248(\mathrm{~m}), 1060(\mathrm{~s})$, 1040 (s), 1000 (s)

HRMS (ESI/QTOF) m/z: [M + H] ${ }^{+}$Calcd for $\mathrm{C}_{25} \mathrm{H}_{36} \mathrm{NO}_{2} \mathrm{Si}^{+}$410.2510; Found 410.2508.

\section{(E)-3-benzyl-5-(1-cyclopropyl-3-(triisopropylsilyl)prop-2-yn-1-ylidene)oxazolidin-2-one (5f)}

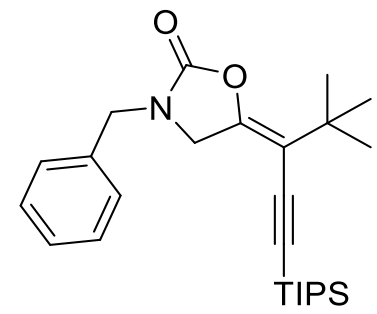

Using $2 \mathrm{f}(81.0 \mathrm{mg}, 0.400 \mathrm{mmol})$. The crude product was purified by column chromatography $\left(\mathrm{SiO}_{2} ; 0-2 \%\right.$ EtOAc in pentane) which afforded (E)-3-benzyl-5-(1-cyclopropyl-3-(triisopropylsilyl)prop-2-yn-1ylidene)oxazolidin-2-one (5f) as a yellow oil ( $74.2 \mathrm{mg}, 0.174 \mathrm{mmol}, 44 \%$ yield)

${ }^{1} \mathrm{H}$ NMR (400 MHz, Chloroform-d) $\delta 7.43-7.21(\mathrm{~m}, 5 \mathrm{H}, \mathrm{ArH}), 4.48\left(\mathrm{~s}, 2 \mathrm{H}, \mathrm{ArCH}_{2}\right), 4.08\left(\mathrm{~s}, 2 \mathrm{H}, \mathrm{NCH} \mathrm{C}_{2} \mathrm{C}\right)$, $1.22\left(\mathrm{~s}, 9 \mathrm{H}, \mathrm{C}\left(\mathrm{CH}_{3}\right)_{3}\right), 1.08-0.95(\mathrm{~m}, 21 \mathrm{H}, \mathrm{TIPS})$.

${ }^{13}$ C NMR (101 MHz, Chloroform-d) $\delta 155.1,147.6,134.7,129.0,128.6,128.4,108.1,103.3,96.6,49.6,48.0$, $33.8,29.6,18.6,11.2$.

IR ( $\left.v_{\max }, \mathrm{cm}^{-1}\right) 664(\mathrm{~s}), 700(\mathrm{~s}), 1041$ (s), 1145 (s), 1415 (s), 1463 (s), 1650 (s), 1795 (s), 2131 (s), 2868 (s), 2958 (s)

HRMS (ESI/QTOF) m/z: [M + Na] ${ }^{+}$Calcd for $\mathrm{C}_{28} \mathrm{H}_{35} \mathrm{NNaO}_{2} \mathrm{Si}^{+}$468.2329; Found 468.2335.

\section{(E)-3-benzyl-5-(1-phenyl-3-(triisopropylsilyl)prop-2-yn-1-ylidene)oxazolidin-2-one (5g)}




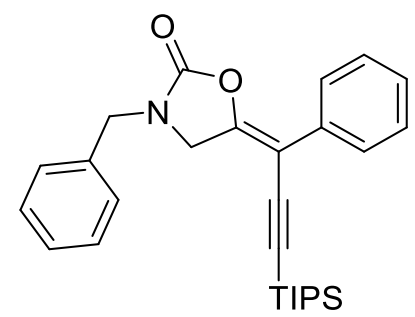

Using $2 \mathrm{~g}(89.0 \mathrm{mg}, 0.400 \mathrm{mmol})$. The crude product was purified by column chromatography $\left(\mathrm{Et}_{3} \mathrm{~N}-\right.$ deactivated $\mathrm{SiO}_{2} ; 0-2 \%$ EtOAc in pentane) which fforded (E)-3-benzyl-5-(1-phenyl-3-(triisopropylsilyl)prop2-yn-1-ylidene)oxazolidin-2-one (5g) as a straw yellow solid (145 $\mathrm{mg}, 0.325 \mathrm{mmol}, 81 \%$ yield). The product was isolated with $7.5 \%$ dibenzylideneacetone which was removed upon preparative $\mathrm{TLC}\left(10 \% \mathrm{Et}_{2} \mathrm{O}\right.$ in pentane) to give an off-white crystalline solid, m.p. $81-82^{\circ} \mathrm{C}$.

${ }^{1} \mathrm{H}$ NMR (400 MHz, Chloroform-d) $\delta 7.80(\mathrm{~m}, 2 \mathrm{H}, \mathrm{ArH}), 7.44-7.21(\mathrm{~m}, 8 \mathrm{H}, \mathrm{ArH}), 4.55(\mathrm{~s}, 2 \mathrm{H}, \mathrm{ArCH}), 4.31$ $\left(\mathrm{s}, 2 \mathrm{H}, \mathrm{NCH}_{2} \mathrm{C}=\mathrm{C}\right), 1.08-1.03(\mathrm{~m}, 21 \mathrm{H}, \mathrm{TIPS})$

${ }^{13} \mathrm{C}$ NMR (101 MHz, Chloroform-d) $\delta$ 154.8, 149.2, 134.4, 132.7, 129.1, 128.5, 128.5, 128.3, 127.7, 127.5, $102.1,99.9,96.9,49.7,48.1,18.6,11.2$.

IR $\left(v_{\max }, \mathrm{cm}^{-1}\right) 2941(\mathrm{~m}), 2863(\mathrm{~m}), 2140(\mathrm{w}), 1802(\mathrm{~s}), 1647(\mathrm{~m}), 1463(\mathrm{~m}), 1417(\mathrm{~m}), 1261(\mathrm{~m}), 1129(\mathrm{~m})$, $1034(\mathrm{~s})$

HRMS (ESI/QTOF) m/z: [M + Na] ${ }^{+}$Calcd for $\mathrm{C}_{28} \mathrm{H}_{35} \mathrm{NNaO}_{2} \mathrm{Si}^{+} 468.2329$; Found 468.2335.

Cambridge Crystallographic Data Centre entry - 1905359

\section{(E)-3-methyl-5-(1-phenyl-3-(triisopropylsilyl)prop-2-yn-1-ylidene)oxazolidin-2-one (5h)}<smiles></smiles>

Using $2 \mathrm{~h}(58.0 \mathrm{mg}, 0.400 \mathrm{mmol})$. The crude product was purified by column chromatography ( $\mathrm{Et}_{3} \mathrm{~N}-$ deactivated $\mathrm{SiO}_{2} ; 0-4 \%$ EtOAc in pentane) affording the named compound (E)-3-methyl-5-(1-phenyl-3(triisopropylsilyl)prop-2-yn-1-ylidene)oxazolidin-2-one (5h) as an amorphous orange solid (97.9 mg, 0.265 $\mathrm{mmol}, 66 \%$ yield).

${ }^{1} \mathrm{H}$ NMR $(400 \mathrm{MHz}$, Chloroform-d) $\delta 7.83(\mathrm{~d}, J=8.0 \mathrm{~Hz}, 2 \mathrm{H}, \operatorname{ArH}), 7.36(\mathrm{t}, J=7.5 \mathrm{~Hz}, 2 \mathrm{H}, \operatorname{ArH}), 7.26(\mathrm{~m}, 1 \mathrm{H}$, $\operatorname{ArH}), 4.49\left(\mathrm{~s}, 2 \mathrm{H}, \mathrm{NCH}_{2} \mathrm{C}=\mathrm{C}\right), 3.05\left(\mathrm{~s}, 3 \mathrm{H}, \mathrm{CH}_{3}\right), 1.20-1.07(\mathrm{~m}, 21 \mathrm{H}, \mathrm{TIPS})$.

${ }^{13} \mathrm{C}$ NMR (101 MHz, Chloroform-d) $\delta$ 155.0, 148.9, 132.8, 128.3, 127.7, 127.5, 102.0, 99.8, 97.0, 52.2, 30.6, 18.7, 11.2 .

IR $\left(v_{\max }, \mathrm{cm}^{-1}\right) 2945(\mathrm{~m}), 2862(\mathrm{~m}), 2147(\mathrm{w}), 1808(\mathrm{~s}), 1649(\mathrm{~m}), 1463(\mathrm{~m}), 1403(\mathrm{~m}), 1269(\mathrm{~m}), 1132(\mathrm{~s})$, $1080(\mathrm{~s}), 1006(\mathrm{~s}), 883(\mathrm{~m}), 765(\mathrm{~m}), 660(\mathrm{~s})$ 
HRMS (APPI/LTQ-Orbitrap) m/z: [M] ${ }^{+}$Calcd for $\mathrm{C}_{22} \mathrm{H}_{31} \mathrm{NO}_{2} \mathrm{Si}^{+}$369.2119; Found 369.2116.

(E)-3-benzyl-5-(1-(4-methoxyphenyl)-3-(triisopropylsilyl)prop-2-yn-1-ylidene)oxazolidin-2-one (5i)<smiles>COc1ccc(C(C#C[In])=C2CN(Cc3ccccc3)C(=O)O2)cc1</smiles>

Using $2 \mathbf{i}$ (101 $\mathrm{mg}, 0.400 \mathrm{mmol})$. The crude product was purified by column chromatography ( $\mathrm{Et}_{3} \mathrm{~N}-$ deactivated $\mathrm{SiO}_{2} ; 0-2 \%$ EtOAc in pentane) (E)-3-benzyl-5-(1-(4-methoxyphenyl)-3-(triisopropylsilyl)prop-2yn-1-ylidene)oxazolidin-2-one (5i) as a pale amorphous orange solid (129 mg, $0.271 \mathrm{mmol}, 68 \%$ yield).

${ }^{1} \mathrm{H}$ NMR (400 MHz, Chloroform-d) $\delta 7.80-7.71(\mathrm{~m}, 2 \mathrm{H}, \mathrm{ArH}), 7.44-7.27(\mathrm{~m}, 5 \mathrm{H}, \mathrm{ArH}), 6.94-6.85(\mathrm{~m}, 2 \mathrm{H}$, $\operatorname{ArH}), 4.55\left(\mathrm{~s}, 2 \mathrm{H}, \mathrm{ArCH}_{2}\right), 4.30\left(\mathrm{~s}, 2 \mathrm{H}, \mathrm{NCH}_{2} \mathrm{C}=\mathrm{C}\right), 3.82\left(\mathrm{~s}, 3 \mathrm{H}, \mathrm{CH}_{3}\right), 1.10-1.00$ (s, 21H, TIPS).

${ }^{13} \mathrm{C}$ NMR (101 MHz, Chloroform-d) $\delta$ 158.8, 154.9, 147.7, 134.4, 129.0, 128.9, 128.5, 128.4, 125.3, 113.6, $102.3,99.4,96.6,55.2,49.6,48.0,18.6,11.2$.

IR $\left(v_{\max }, \mathrm{cm}^{-1}\right) 2946(\mathrm{~m}), 2867(\mathrm{~m}), 2143(\mathrm{~m}), 1790(\mathrm{~s}), 1647(\mathrm{~m}), 1512(\mathrm{~s}), 1249(\mathrm{~s}), 1182(\mathrm{~s}), 1031(\mathrm{~s}), 836$ (s)

HRMS (ESI/QTOF) m/z: [M + Na] ${ }^{+}$Calcd for $\mathrm{C}_{29} \mathrm{H}_{37} \mathrm{NNaO}_{3} \mathrm{Si}^{+}$498.2435; Found 498.2443.

\section{(E)-3-benzyl-5-(1-(4-(trifluoromethyl)phenyl)-3-(triisopropylsilyl)prop-2-yn-1-ylidene)oxazolidin-2-one}

(5i)<smiles>O=C1OC(=C(C#C[In])c2ccc(C(F)(F)F)cc2)CN1Cc1ccccc1</smiles>

Using $2 \mathbf{j}$ (116 $\mathrm{mg}, 0.400 \mathrm{mmol})$. The crude product was purified by column chromatography ( $\mathrm{Et}_{3} \mathrm{~N}-$ deactivated $\mathrm{SiO}_{2} ; \quad 0-2 \% \quad \mathrm{EtOAC}$ in pentane) (E)-3-benzyl-5-(1-(4-(trifluoromethyl)phenyl)-3(triisopropylsilyl)prop-2-yn-1-ylidene)oxazolidin-2-one (5j) as a red oil (105 mg, 0.204 mmol, 51\% yield).

${ }^{1} \mathrm{H}$ NMR (400 MHz, Chloroform-d) $\delta 7.92(\mathrm{~d}, J=8.2 \mathrm{~Hz}, 2 \mathrm{H}, \mathrm{ArH}), 7.61$ (d, J = 8.3 Hz, 2H, ArH), $7.45-7.27$ $(\mathrm{m}, 5 \mathrm{H}, \mathrm{ArH}), 4.57\left(\mathrm{~s}, 2 \mathrm{H}, \mathrm{ArCH}_{2}\right), 4.33\left(\mathrm{~s}, 2 \mathrm{H}, \mathrm{NCH}_{2} \mathrm{C}=\mathrm{C}\right), 1.07(\mathrm{~m}, 21 \mathrm{H}, \mathrm{TIPS})$.

${ }^{13} \mathrm{C}$ NMR (101 MHz, Chloroform-d) $\delta$ 154.3, 151.0, 136.3, 134.2, 129.1, 128.6, 128.5, 127.8, 125.2 (q, J = 3.9 $\mathrm{Hz}), 124.1$ (q, $J=272.0 \mathrm{~Hz}$ ), 101.2, 98.8, 97.8, 49.8, 48.1, 18.6, 11.1. One carbon not resolved.

${ }^{19} \mathrm{~F}$ NMR (376 MHz, Chloroform-d) $\delta-62.5$. 
IR (vmax, cm-1) 662 (s), 882 (s), 1052 (s), 1201 (s), 1247 (s), 1324 (s), 1416 (s), 1468 (s), 1686 (s), 1792 (s), $2148(\mathrm{~s}), 2867(\mathrm{~s}), 2940$ (s)

HRMS (ESI/QTOF) m/z: [M + Na] $]^{+}$Calcd for $\mathrm{C}_{29} \mathrm{H}_{34} \mathrm{~F}_{3} \mathrm{NNaO}_{2} \mathrm{Si}^{+}$536.2203; Found 536.2195.

\section{(E)-5-(1-(3-bromophenyl)-3-(triisopropylsilyl)prop-2-yn-1-ylidene)-3-phenyloxazolidin-2-one}

(5k)<smiles></smiles>

Following general procedure B using $2 \mathbf{k}(120 \mathrm{mg}, 0.400 \mathrm{mmol})$. The crude product was dry loaded onto deactivated $\mathrm{SiO}_{2}$ and purified by column chromatography $\left(\mathrm{SiO}_{2} ; 0-3 \%\right.$ EtOAc in pentane) which afforded (E)-5-(1-(3-bromophenyl)-3-(triisopropylsilyl)prop-2-yn-1-ylidene)-3-phenyloxazolidin-2-one as a brown amorphous solid (124 $\mathrm{mg}, 0.243 \mathrm{mmol}, 61 \%$ yield)

${ }^{1} \mathrm{H}$ NMR $(400 \mathrm{MHz}$, Chloroform-d) $\delta 7.94(\mathrm{t}, J=1.9 \mathrm{~Hz}, 1 \mathrm{H}, \mathrm{ArH}), 7.76(\mathrm{dt}, J=7.9,1.3 \mathrm{~Hz}, 1 \mathrm{H}, \mathrm{ArH}), 7.44-$ $7.27(\mathrm{~m}, 6 \mathrm{H}, \mathrm{ArH}), 7.22(\mathrm{t}, \mathrm{J}=7.9 \mathrm{~Hz}, 1 \mathrm{H}, \mathrm{ArH}), 4.56\left(\mathrm{~s}, 2 \mathrm{H}, \mathrm{ArCH}_{2}\right), 4.30\left(\mathrm{~s}, 2 \mathrm{H}, \mathrm{NCH}_{2} \mathrm{C}=\mathrm{C}\right), 1.10-1.04(\mathrm{~m}$, $21 \mathrm{H}, \mathrm{TIPS})$.

${ }^{13} \mathrm{C}$ NMR $(101 \mathrm{MHz}$, Chloroform-d) $\delta 154.4,150.2,134.8,134.3,130.6,130.4,129.8,129.1,128.6,128.5$, $126.2,122.4,101.3,98.6,97.7,49.7,48.1,18.6,11.2$.

IR ( $\left.v_{\max }, \mathrm{cm}^{-1}\right) 702(\mathrm{~s}), 1033$ (s), 1253 (s), 1416 (s), 1464 (s), 1649 (s), 1803 (s), 2147 (s), 2866 (s), $2947(\mathrm{~s})$. HRMS (ESI/QTOF) m/z: [M + H] ${ }^{+}$Calcd for $\mathrm{C}_{28} \mathrm{H}_{35}{ }^{79} \mathrm{BrNO}_{2} \mathrm{Si}^{+}$524.1615; Found 524.1601.

\section{(E)-3-benzyl-5-(1-(3,5-dimethylphenyl)-3-(triisopropylsilyl)prop-2-yn-1-ylidene)oxazolidin-2-one}

(5I)<smiles></smiles>

Using 21 (100 mg, $0.400 \mathrm{mmol})$. The crude product was dry loaded onto deactivated $\mathrm{SiO}_{2}$ and purified by column chromatography $\left(\mathrm{SiO}_{2} ; 0-3 \%\right.$ EtOAc in pentane) which afforded (E)-3-benzyl-5-(1-(3,5dimethylphenyl)-3-(triisopropylsilyl)prop-2-yn-1-ylidene)oxazolidin-2-one (5I) as an orange amorphous solid (151 $\mathrm{mg}, 0.331 \mathrm{mmol}, 80 \%$ yield) 
${ }^{1} \mathrm{H}$ NMR (400 MHz, Chloroform-d) $\delta 7.43(\mathrm{~s}, 2 \mathrm{H}, \mathrm{ArH}), 7.42-7.29(\mathrm{~m}, 5 \mathrm{H}, \mathrm{ArH}), 6.94-6.86(\mathrm{~m}, 1 \mathrm{H}, \operatorname{ArH})$, $4.55\left(\mathrm{~s}, 2 \mathrm{H}, \mathrm{ArCH}_{2}\right), 4.30\left(\mathrm{~s}, 2 \mathrm{H}, \mathrm{NCH}_{2} \mathrm{C}=\mathrm{C}\right), 2.32\left(\mathrm{~s}, 6 \mathrm{H}, \mathrm{ArCH}_{3}\right), 1.11-1.02(\mathrm{~m}, 21 \mathrm{H}, \mathrm{TIPS})$.

${ }^{13} \mathrm{C}$ NMR $(101 \mathrm{MHz}$, Chloroform-d) $\delta 154.9,148.8,137.7,134.5,132.5,129.2,129.0,128.5,128.4,125.5$, $102.5,100.0,96.6,49.7,48.0,21.4,18.6,11.2$.

IR $\left(v_{\max }, \mathrm{cm}^{-1}\right) 674(\mathrm{~m}), 702(\mathrm{~s}), 851(\mathrm{~m}), 884(\mathrm{~m}), 1037(\mathrm{~s}), 1133(\mathrm{~m}), 1236(\mathrm{~m}), 1415(\mathrm{~m}), 1463(\mathrm{~m}), 1645$ $(\mathrm{m}), 1800(\mathrm{~s}), 2142(\mathrm{w}), 2866(\mathrm{~m}), 2942(\mathrm{~m})$.

HRMS (ESI/QTOF) m/z: [M+ H] ${ }^{+}$Calcd for $\mathrm{C}_{30} \mathrm{H}_{40} \mathrm{NO}_{2} \mathrm{Si}^{+}$474.2823; Found 474.2822.

\section{(Z)-3-benzyl-5-(1-(thiophen-2-yl)-3-(triisopropylsilyl)prop-2-yn-1-ylidene)oxazolidin-2-one (5m)}<smiles></smiles>

Using $2 \mathrm{~m}$ ( $91.0 \mathrm{mg}, 0.400 \mathrm{mmol})$. The crude product was dry loaded onto deactivated $\mathrm{SiO}_{2}$ and purified by column chromatography $\left(\mathrm{SiO}_{2} ; 0-3 \%\right.$ EtOAc in pentane) which afforded (Z)-3-benzyl-5-(1-(thiophen-2-yl)3-(triisopropylsilyl)prop-2-yn-1-ylidene)oxazolidin-2-one (5m) as an orange oil (91.4 mg, $0.189 \mathrm{mmol}, 47 \%$ yield).

${ }^{1} \mathrm{H}$ NMR $(400 \mathrm{MHz}$, Chloroform-d) $\delta 7.42-7.25(\mathrm{~m}, 5 \mathrm{H}, \mathrm{ArH}, 2 \mathrm{H}$, thiopheneH), $7.03(\mathrm{dd}, J=5.1,3.7 \mathrm{~Hz}, 1 \mathrm{H}$, thiopheneH), $4.56\left(\mathrm{~s}, 2 \mathrm{H}, \mathrm{ArCH}_{2}\right), 4.28\left(\mathrm{~s}, 2 \mathrm{H}, \mathrm{NCH}_{2} \mathrm{C}=\mathrm{C}\right), 1.12-1.02(\mathrm{~m}, 21 \mathrm{H}, \mathrm{TIPS})$.

${ }^{13} \mathrm{C}$ NMR (101 MHz, Chloroform-d) $\delta 154.4,147.0,136.1,134.4,129.1,128.5,128.4,127.1,126.1,125.6$, $100.5,96.9,95.4,48.9,48.1,18.6,11.2$.

IR (vmax, cm-1) $2942(\mathrm{~m}), 2864(\mathrm{~m}), 2148(\mathrm{w}), 1796(\mathrm{~s}), 1651(\mathrm{~m}), 1462(\mathrm{~m}), 1414(\mathrm{~m}), 1253(\mathrm{~s}), 1097(\mathrm{~m})$, $1039(\mathrm{~s}), 882(\mathrm{~m}), 744(\mathrm{~m}), 697(\mathrm{~s})$, 665(s).

HRMS (ESI/QTOF) m/z: [M + H] ${ }^{+}$Calcd for $\mathrm{C}_{26} \mathrm{H}_{34} \mathrm{NO}_{2} \mathrm{SSi}^{+}$452.2074; Found 452.2071. 
(E)-3-benzyl-5-(1-(p-tolyl)ethylidene)oxazolidin-2-one (9) and (Z)-3-benzyl-5-ethylideneoxazolidin-2one (10)

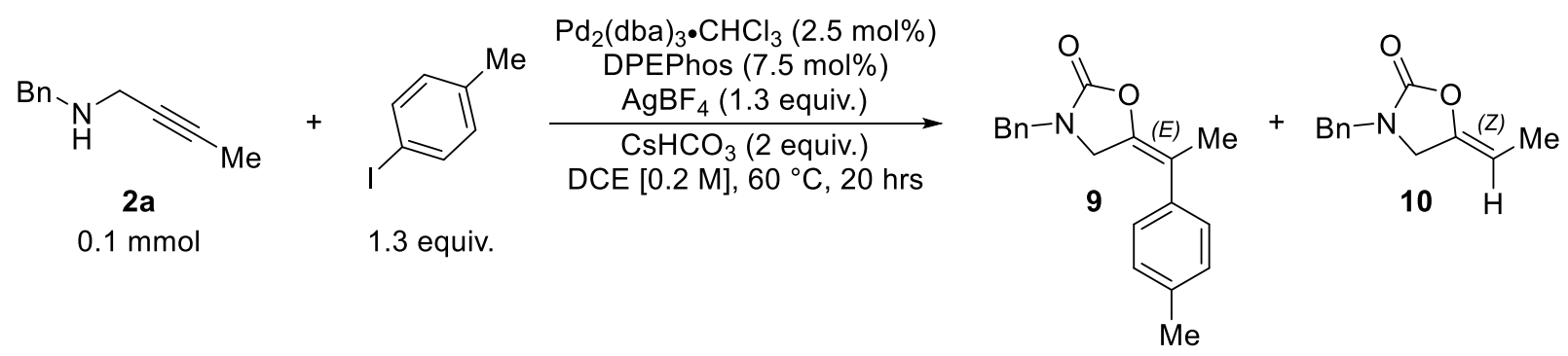

A vial was charged with $\mathrm{Pd}_{2}\left(\mathrm{dba}_{3} \cdot \mathrm{CHCl}_{3}(2.6 \mathrm{mg}, 2.5 \mu \mathrm{mol}, 2.5 \mathrm{~mol} \%)\right.$, DPEPhos (4.4 mg, $7.5 \mu \mathrm{mol}, 7.5$ mol\%), $\mathrm{AgBF}_{4}$ (25 mg, $0.13 \mathrm{mmol}, 1.3$ equiv.) and $\mathrm{CsHCO}_{3}(38 \mathrm{mg}, 2.0 \mathrm{mmol}, 2.0$ equiv.). The vial was sealed and purged with argon. To this was added DCE $(0.3 \mathrm{~mL})$ and the mixture was heated at $60{ }^{\circ} \mathrm{C}$ for 2-3 minutes, after which it took on a black colour. N-benzylbut-2-yn-1-amine (2a) (16 mg, $0.1 \mathrm{mmol}, 1.0$ equiv.) and a solution of 4-lodotoluene ( $28 \mathrm{mg}, 0.13 \mathrm{mmol}, 1.3$ equiv.) in DCE (200 $\mu \mathrm{L})$ were added directly to the reaction mixture and left to stir at $60^{\circ} \mathrm{C}$ for 20 hours. After this time, the reaction mixture was removed from heating, cooled to room temperature, unsealed, diluted with pentane $(2 \mathrm{~mL})$ and passed through a small plug of silica gel (deactivated by $\mathrm{Et}_{3} \mathrm{~N}$ ) rinsing with $10 \%$ EtOAc in pentane $(4 \mathrm{~mL})$ and concentrated by rotary evaporation. The crude material was purified by preparative TLC (15\% EtOAC in pentane) to afford a mixture of the named compounds $\mathbf{9}$ and $\mathbf{1 0}$ in a 1.5:1 ratio as a straw yellow oil (13.5 mg, $53.0 \mu \mathrm{mol} 53 \%$ yield) containing some impurities.

${ }^{1} \mathrm{H}$ NMR (400 MHz, Chloroform-d; as a 1.5:1 mixture of $\underline{9}$ and 10$) \delta 7.41-7.21(\mathrm{~m}, \underline{5 \mathrm{H}, \mathrm{ArH}} ; \underline{5 \mathrm{H}}, \mathrm{ArH})$, $7.12(\mathrm{~d}, J=7.9 \mathrm{~Hz}, \underline{2 \mathrm{H}}, \mathrm{ArH}), 7.05-7.00(\mathrm{~m}, \underline{2 \mathrm{H}}, \mathrm{ArH}), 4.55$ (qt, J = 6.9, $2.2 \mathrm{~Hz}, \underline{1 \mathrm{H}}, \mathrm{C}=\mathrm{C}=\mathrm{CH}), 4.46(\mathrm{~s}, 2 \mathrm{H}$,

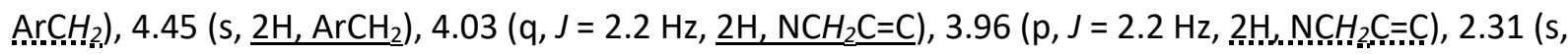
$\left.\underline{3 \mathrm{H}, \mathrm{ArCH}_{3}}\right), 2.07\left(\mathrm{t}, \mathrm{J}=2.2 \mathrm{~Hz}, \underline{3 \mathrm{H}, \mathrm{CH}_{3}}\right), 1.67\left(\mathrm{dt}, \mathrm{J}=7.0,2.3 \mathrm{~Hz}, 3 \mathrm{H}, . . \mathrm{CH}_{3}\right)$.

${ }^{13} \mathrm{C} \mathrm{NMR}$ (101 MHz, Chloroform- $d$; as a 1.5:1 mixture of $\underline{\mathbf{9}}$ and $\mathbf{1 0}$, some signals could not be attributed) $\delta$ 156.0, 155.9, 141. $6, \underline{138.1}, \underline{136.9}, \underline{135.9}, 135.2, \underline{129.3}, 128.9,128.1,128.1,128.1,127.1, \underline{112.1}, 97.6$, $\underline{47.9}, 47.9, \underline{47.4}, 47.1, \underline{21.1}, \underline{16.5}, 9.9 .9$.

IR ( $\left.v_{\max }, \mathrm{cm}^{-1}\right) 3031(w), 2921(w), 2863(w), 1777(s), 1716(\mathrm{~m}), 1422(\mathrm{~m}), 1258(\mathrm{~m}), 1083(\mathrm{~s}), 1062(\mathrm{~s})$, $821(\mathrm{~m}), 754(\mathrm{~m}), 703(\mathrm{~s})$.

HRMS (nanochip-ESI/LTQ-Orbitrap) m/z: $[\mathrm{M}+\mathrm{H}]^{+}$Calcd for $\mathrm{C}_{19} \mathrm{H}_{20} \mathrm{NO}_{2}{ }^{+}$294.1489; Found 294.1490.

HRMS (nanochip-ESI/LTQ-Orbitrap) m/z: $[\mathrm{M}+\mathrm{H}]^{+}$Calcd for $\mathrm{C}_{12} \mathrm{H}_{13} \mathrm{NO}_{2}{ }^{+}$204.1019; Found 204.1020.

The spectra of 9 closely resembles similar structure in literature. ${ }^{6}$

\section{References.}

(1) Zalesskiy, S. S.; Ananikov, V. P. Organometallics 2012, 31 (6), 2302-2309.

(2) Shao, Y.; Zhang, F.; Zhang, J.; Zhou, X. Angew. Chemie Int. Ed. 2016, 55 (38), 11485-11489.

(3) Gobé, V.; Retailleau, P.; Guinchard, X. Org. Lett. 2014, 16 (20), 5438-5441.

(4) Greenwood, P. D. G.; Grenet, E.; Waser, J. Chem. - A Eur. J. 2019, 3010-3013. 
(5) Althuon, D.; Rönicke, F.; Fürniss, D.; Quan, J.; Wellhöfer, I.; Jung, N.; Schepers, U.; Bräse, S. Org. Biomol. Chem. 2015, 13 (14), 4226-4230.

(6) García-Domínguez, P.; Fehr, L.; Rusconi, G.; Nevado, C. Chem. Sci. 2016, 7 (6), 3914-3918.

(7) Hashmi, A. S. K.; Häffner, T.; Yang, W.; Pankajakshan, S.; Schäfer, S.; Schultes, L.; Rominger, F.; Frey, W. Chem. - A Eur. J. 2012, 18 (34), 10480-10486.

(8) Kundu, N. G.; Chaudhuri, G. Tetrahedron Lett. 2001, 42, 2883-2886.

(9) Reis, O.; Koyuncu, H.; Esiringu, I.; Sahin, Y.; Gulcan, O. H. A New Method for the Synthesis of Rasagiline. WO2012096635, 2012.

(10) Jiang, M. X. W.; Rawat, M.; Wulff, W. D. J. Am. Chem. Soc. 2004, 126 (19), 5970-5971.

(11) Orcel, U.; Waser, J. Angew. Chemie Int. Ed. 2016, 55 (41), 12881-12885.

\section{Structures determined by X-ray measurement.}
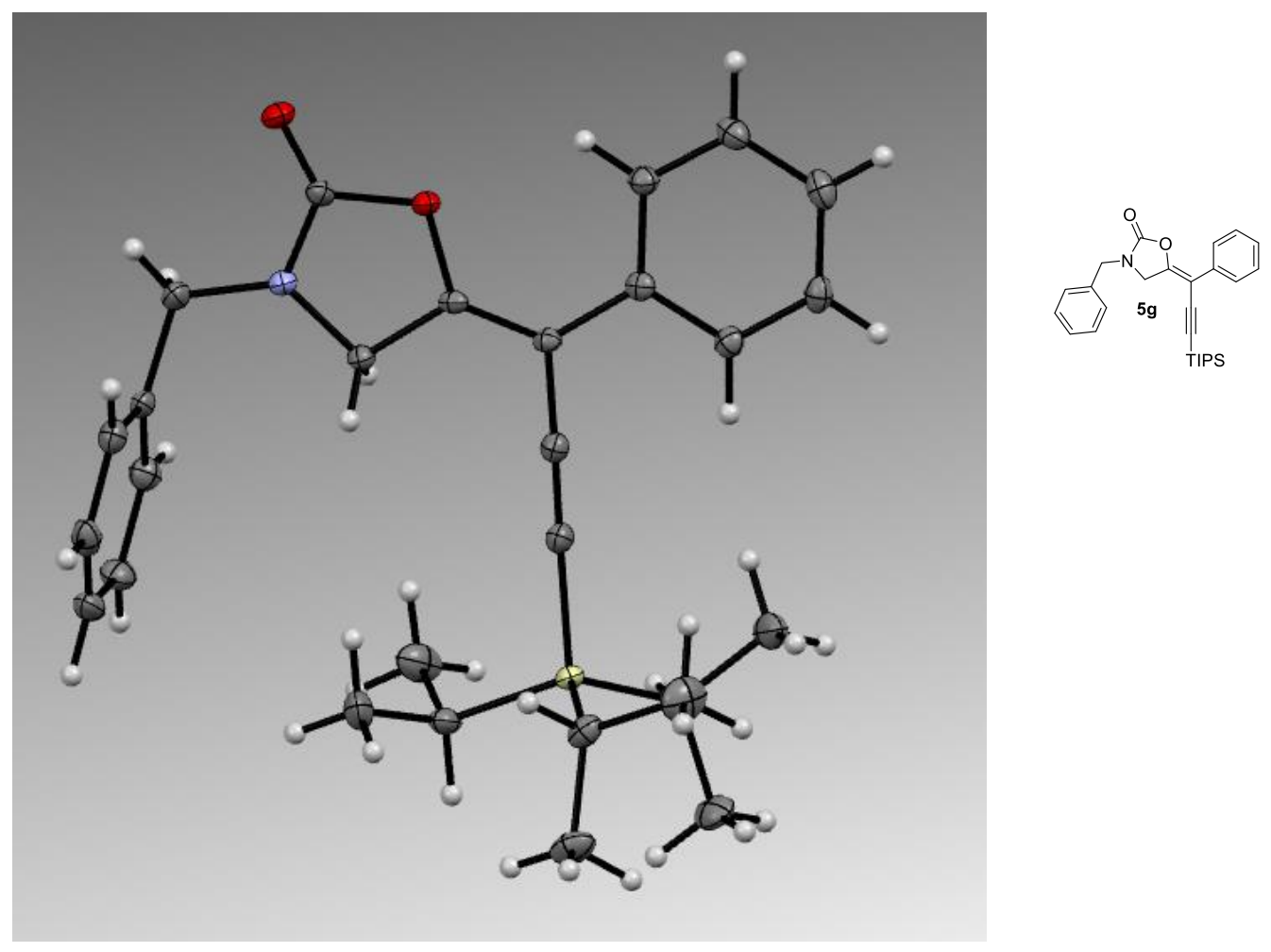

Cambridge Crystallographic Data Centre entry - 1905359

\section{8. ${ }^{1} \mathrm{H} \&{ }^{13} \mathrm{C}$ NMR spectra of new compounds.}


N-benzyl-3-(3-bromophenyl)prop-2-yn-1-amine (2k)

${ }^{1} \mathrm{H}-\mathrm{NMR}$ (400 MHz, chloroform-d)
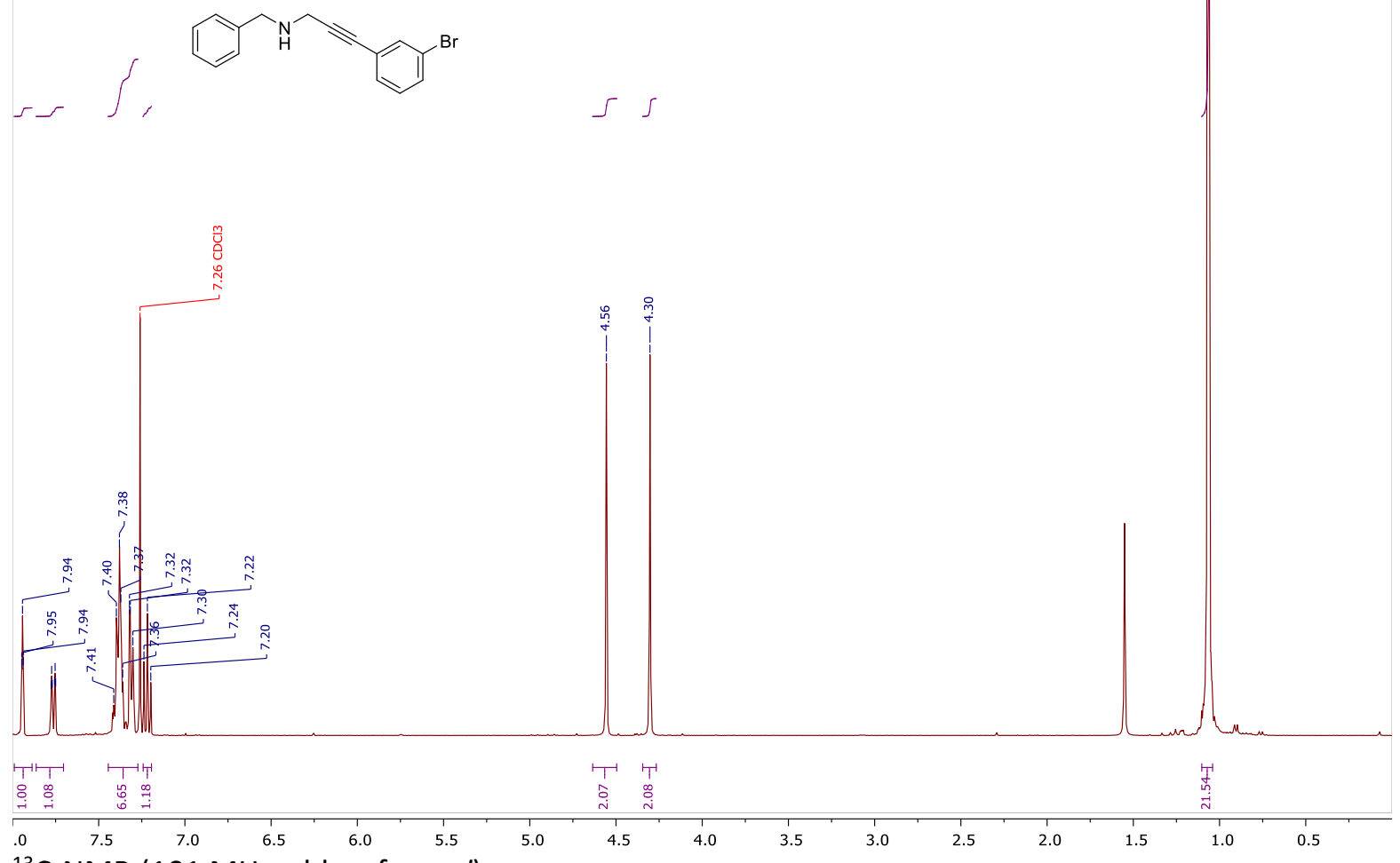

${ }^{13} \mathrm{C}-\mathrm{NMR}$ (101 MHz, chloroform-d)
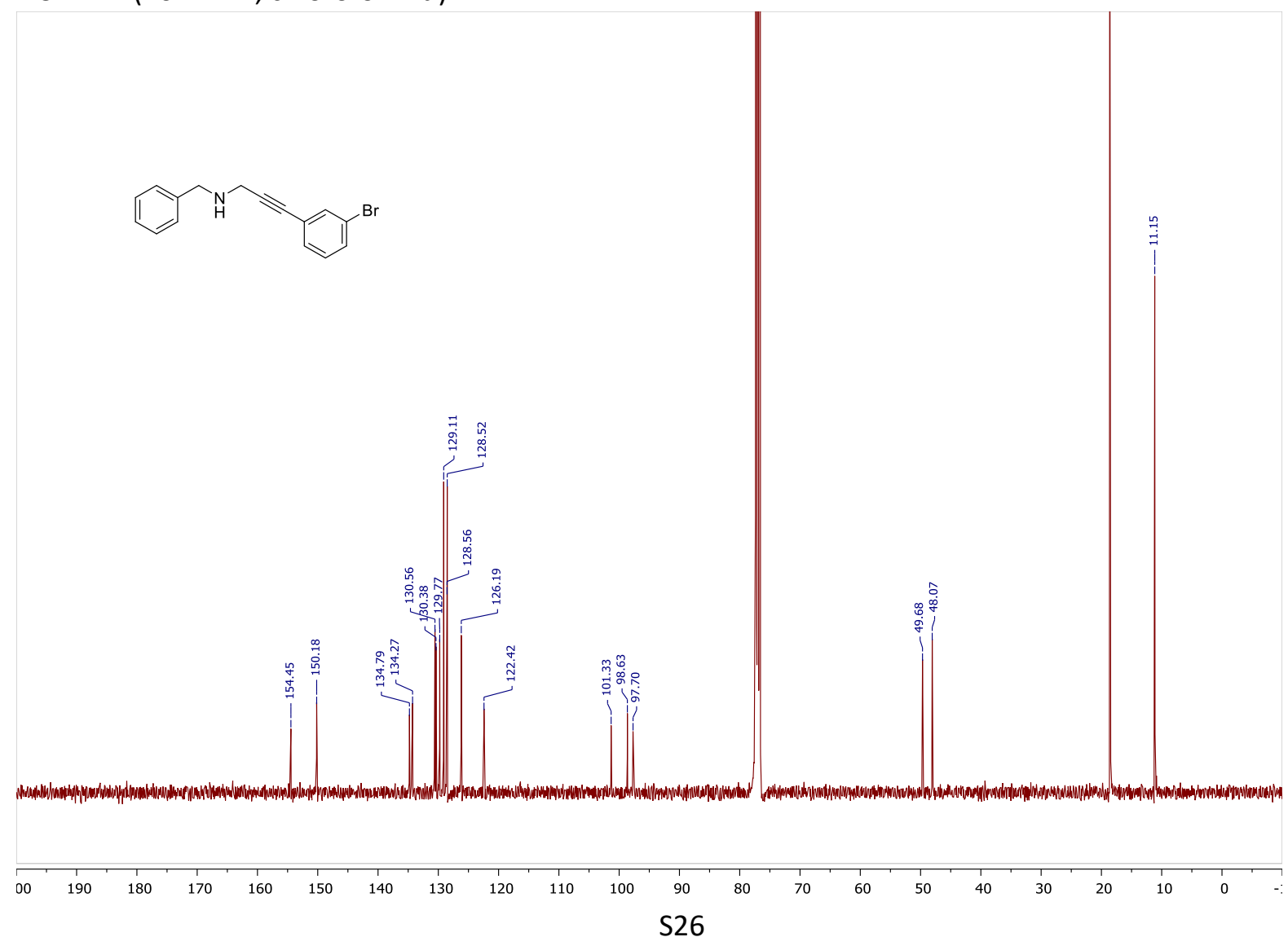
N-benzyl-3-(3-bromophenyl)prop-2-yn-1-amine (2m)

${ }^{1} \mathrm{H}-\mathrm{NMR}$ (400 MHz, chloroform-d)

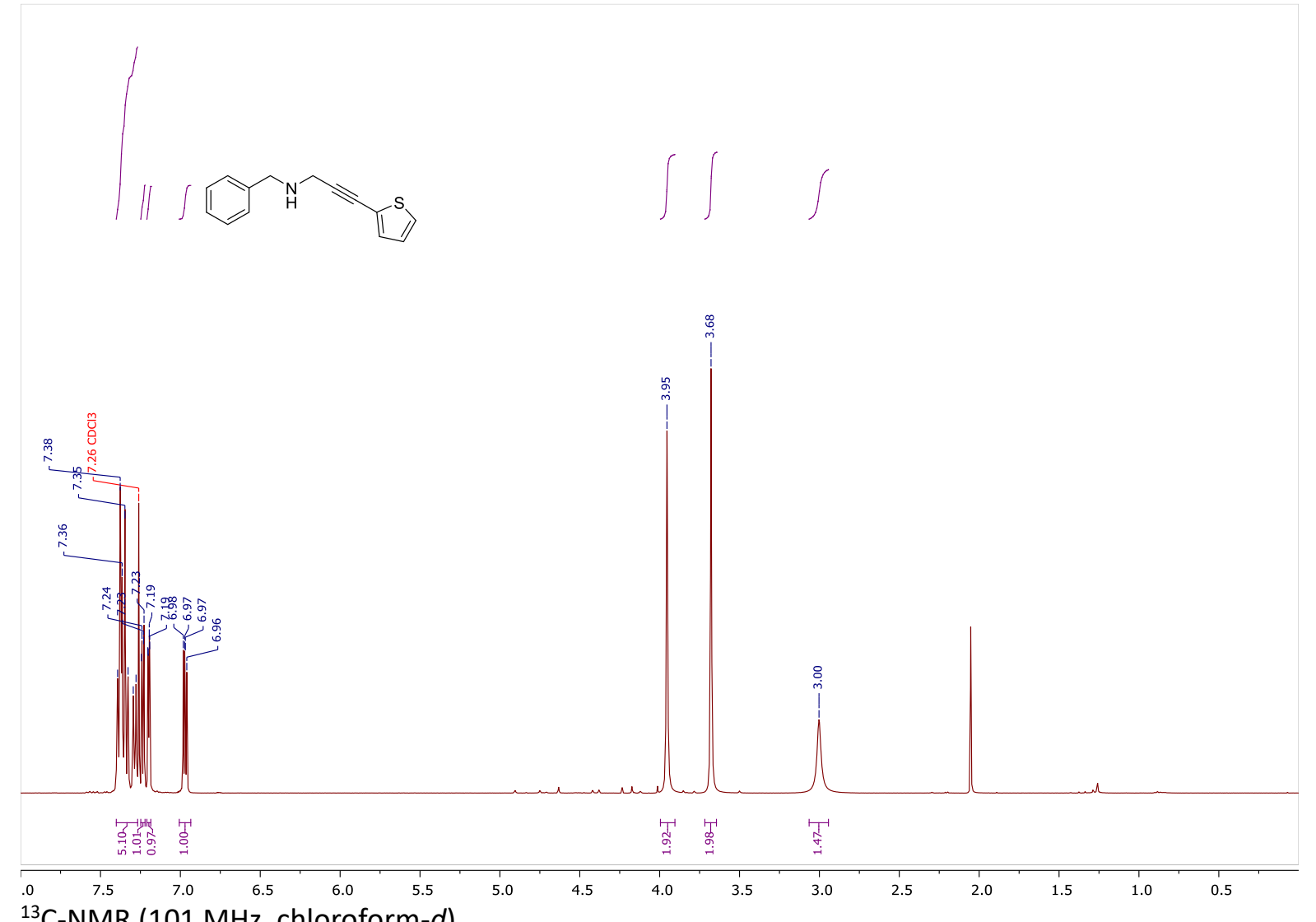

${ }^{13} \mathrm{C}-\mathrm{NMR}(101 \mathrm{MHz}$, chloroform-d)

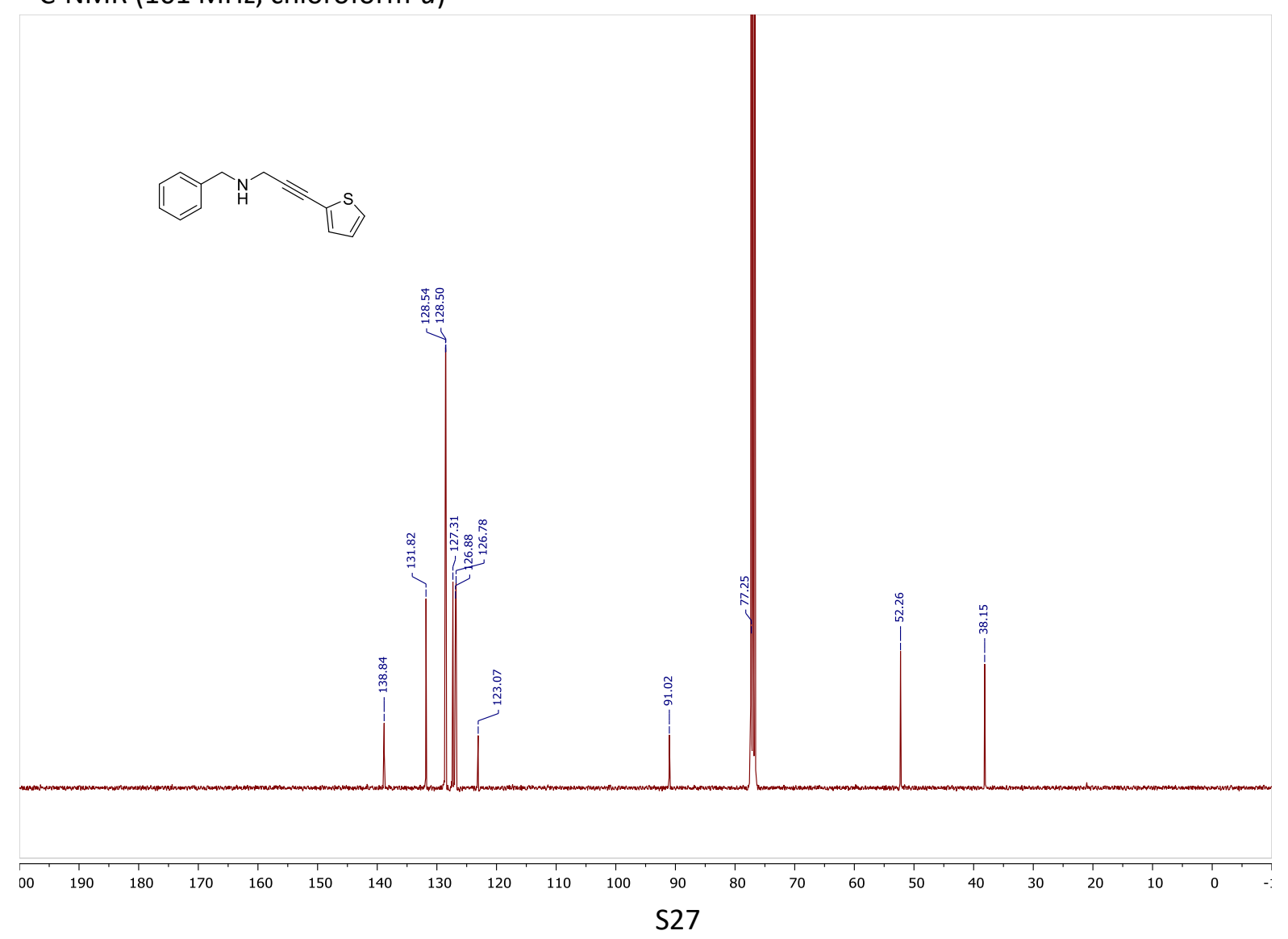


(E)-3-benzyl-5-(4-(triisopropylsilyl)but-3-yn-2-ylidene)oxazolidin-2-one (5a)

${ }^{1} \mathrm{H}-\mathrm{NMR}$ (400 MHz, chloroform-d)

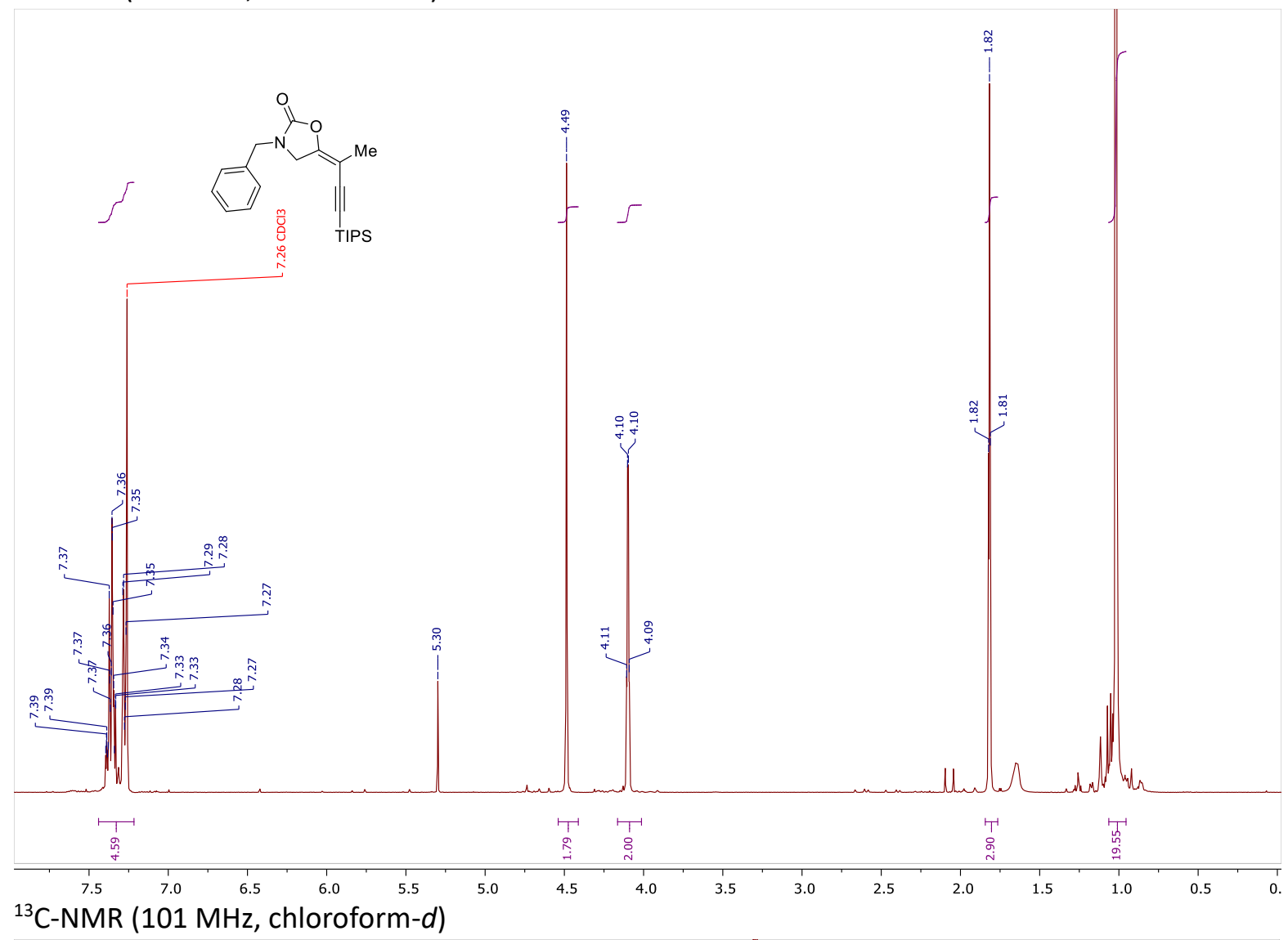

${ }^{13} \mathrm{C}-\mathrm{NMR}$ (101 MHz, chloroform-d)
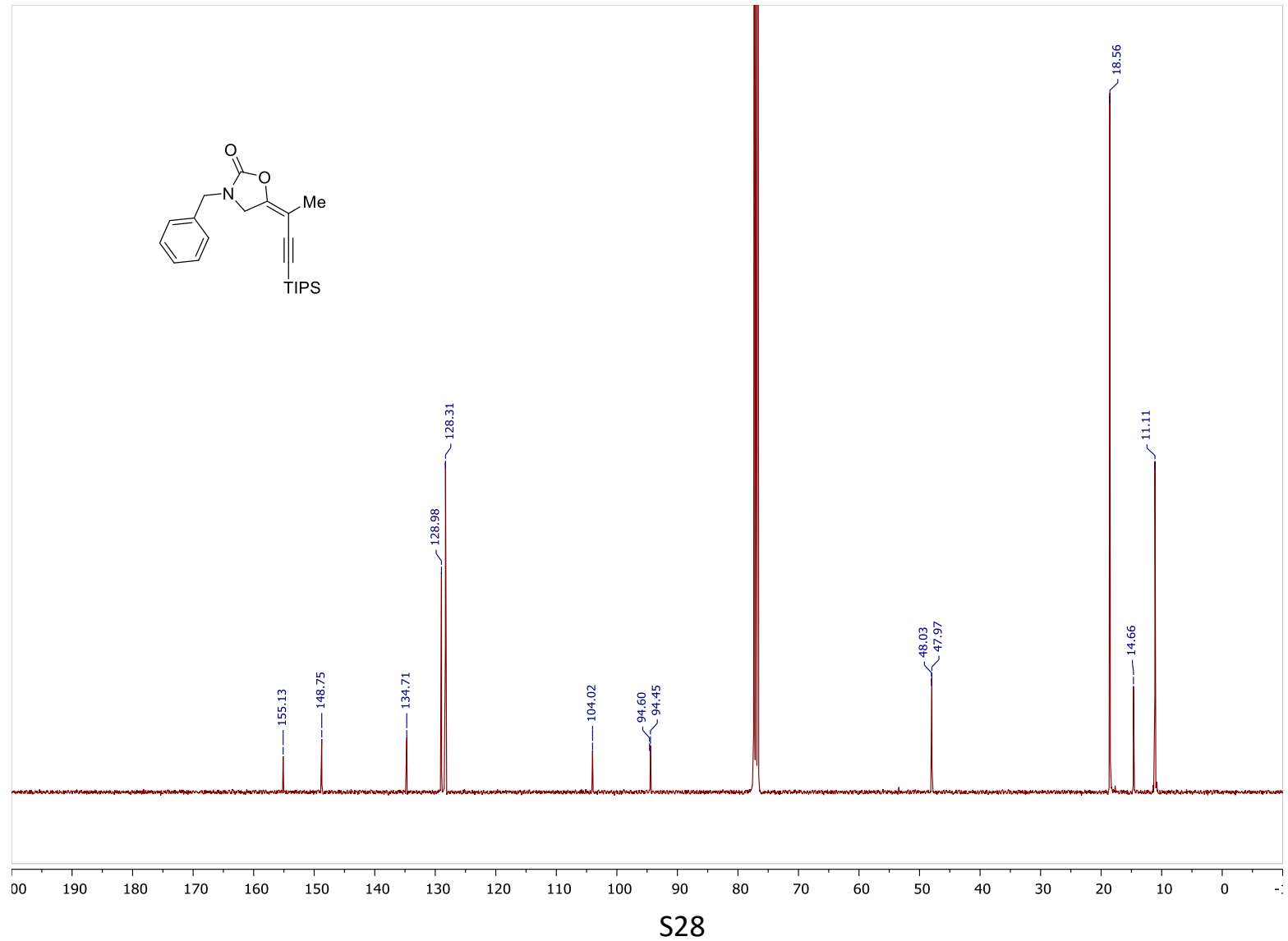
(E)-3-(4-methoxybenzyl)-5-(4-(triisopropylsilyl)but-3-yn-2-ylidene)oxazolidin-2-one (5b)

${ }^{1} \mathrm{H}-\mathrm{NMR}(400 \mathrm{MHz}$, chloroform-d)

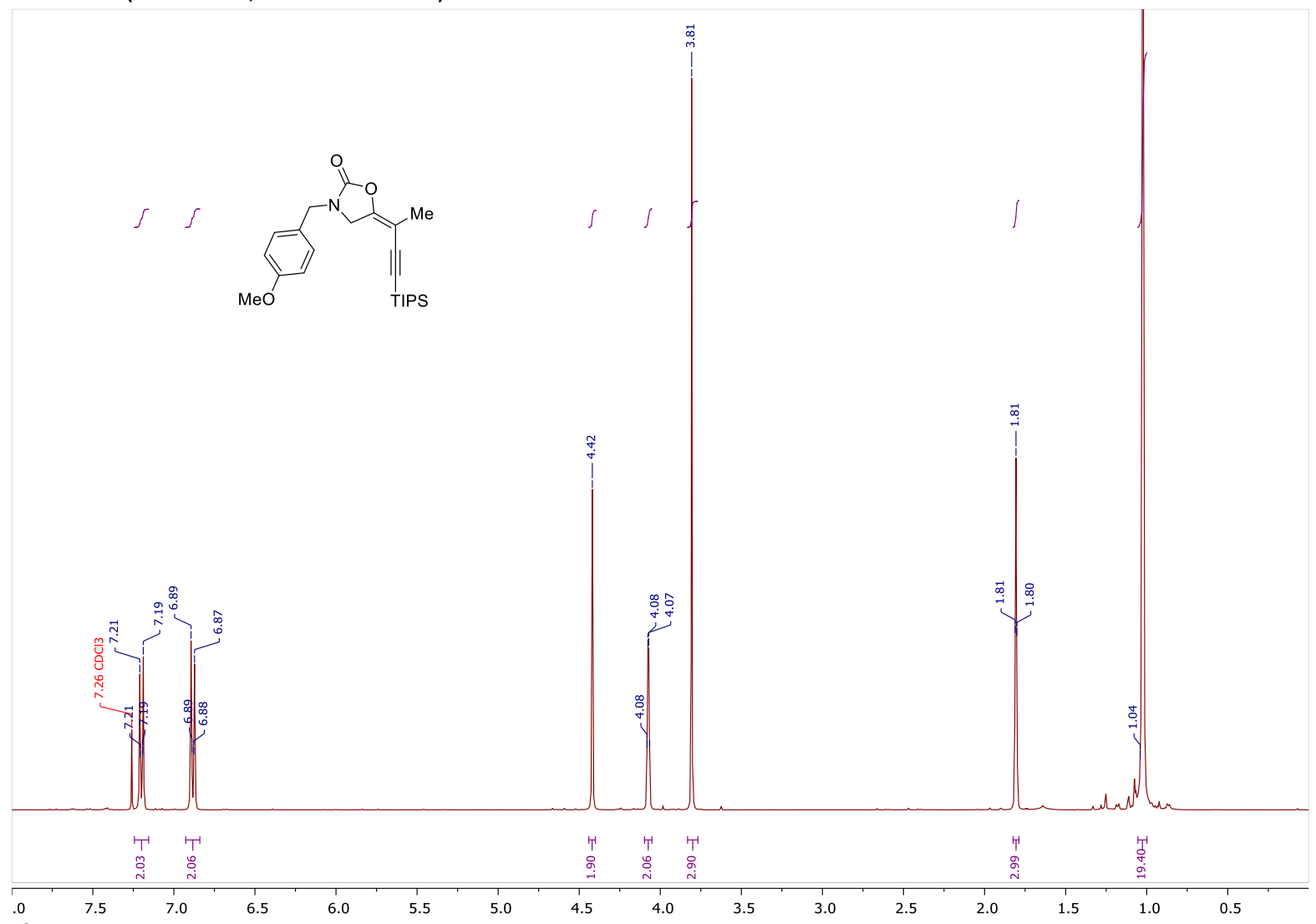

${ }^{13} \mathrm{C}-\mathrm{NMR}(101 \mathrm{MHz}$, chloroform-d)
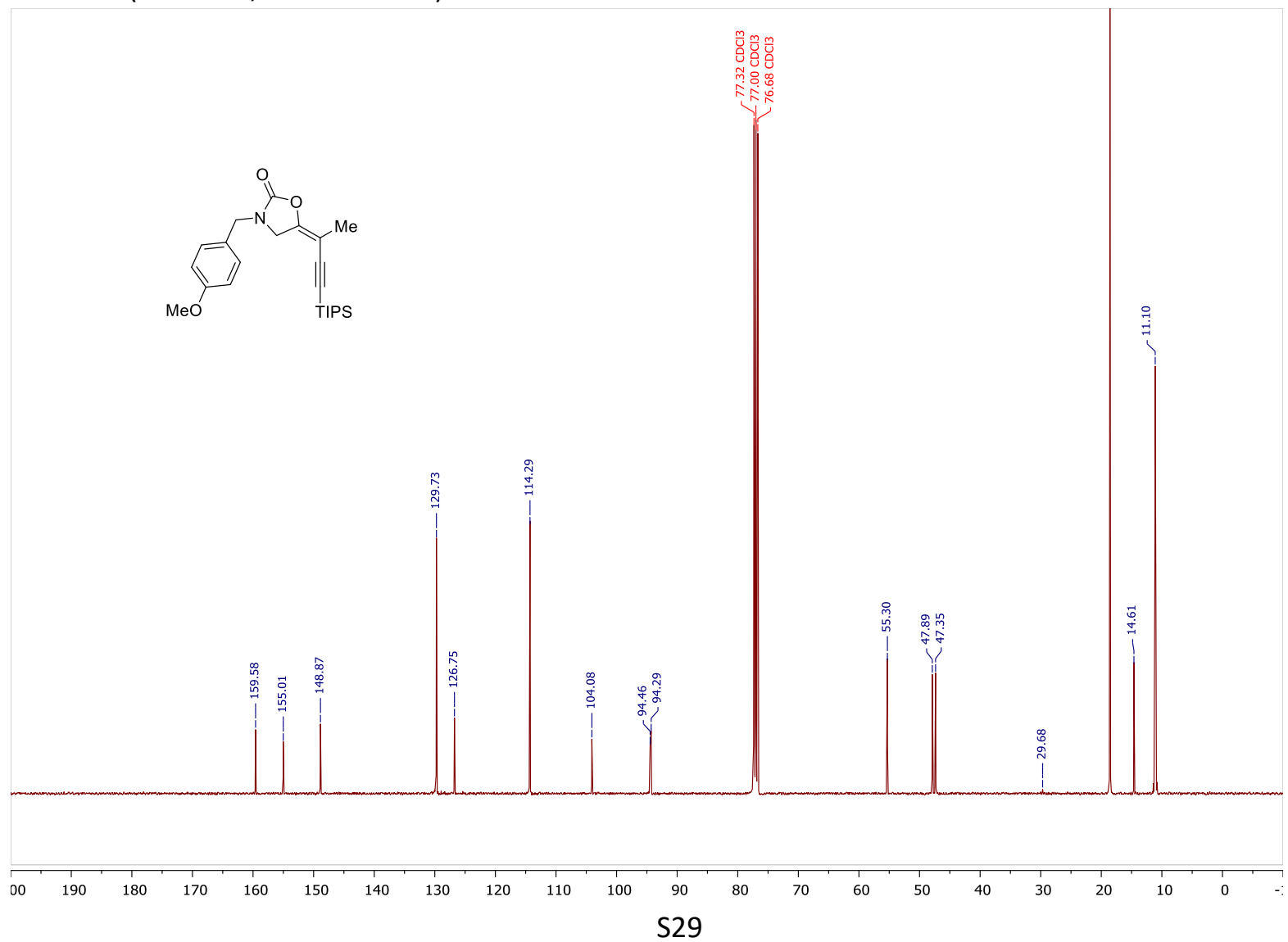
(E)-3-ferrocenylmethyl-5-(4-(triisopropylsilyl)but-3-yn-2-ylidene)oxazolidin-2-one (5c)

${ }^{1} \mathrm{H}-\mathrm{NMR}$ (400 MHz, chloroform-d)
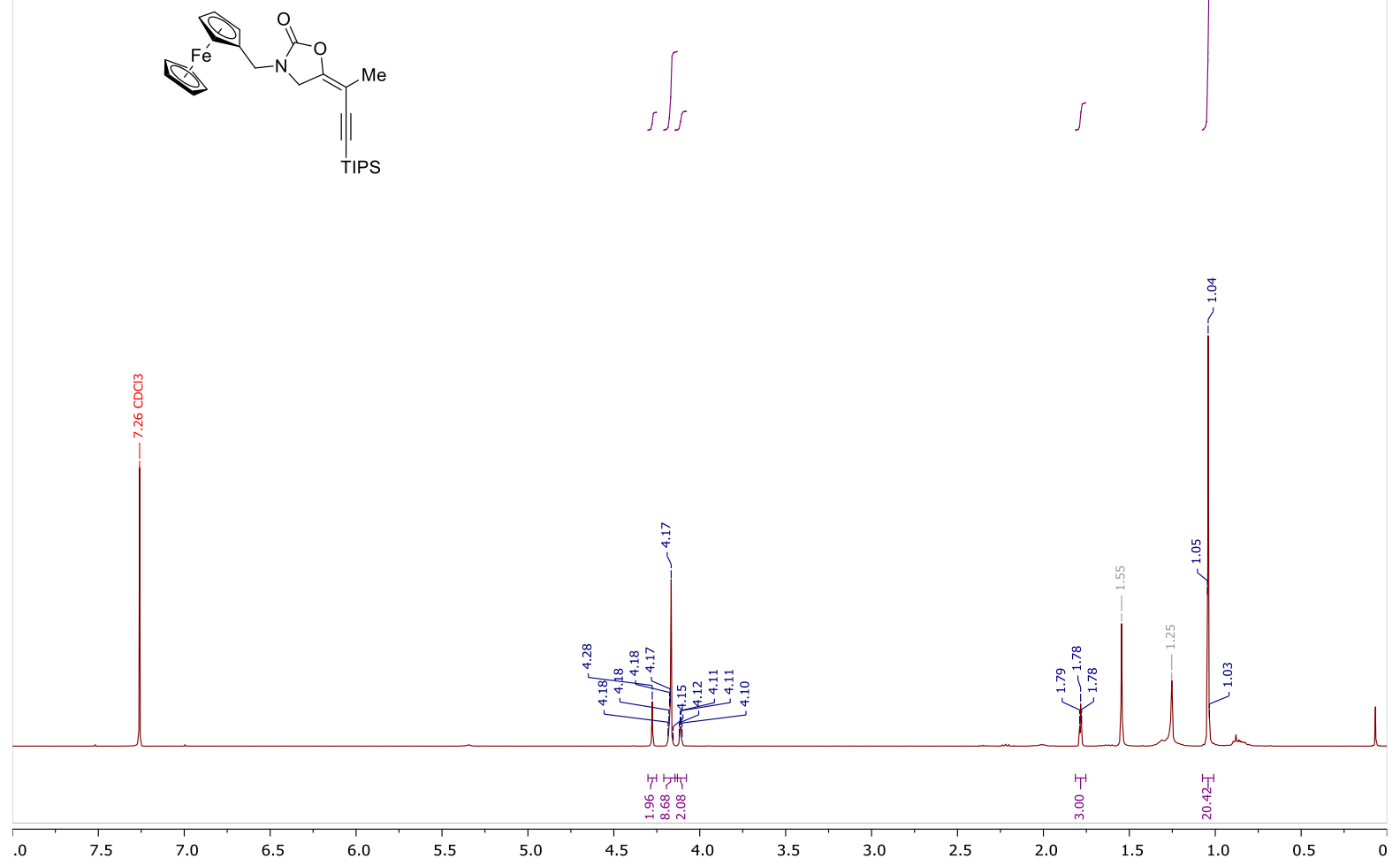

${ }^{13} \mathrm{C}-\mathrm{NMR}$ (101 MHz, chloroform-d)
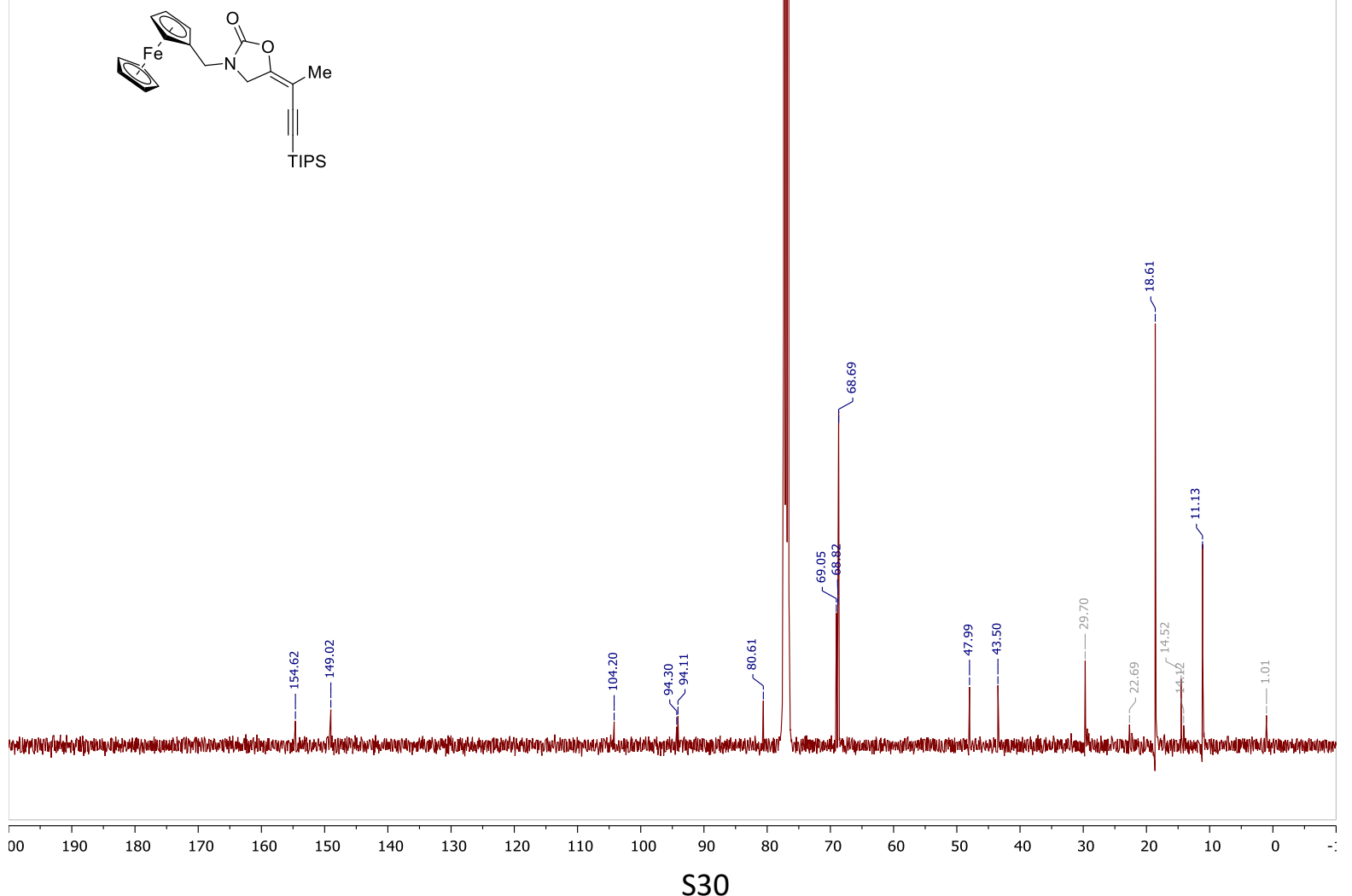
(E)-3-benzyl-5-(1-phenyl-4-(triisopropylsilyl)but-3-yn-2-ylidene)oxazolidin-2-one (5d)

${ }^{1} \mathrm{H}-\mathrm{NMR}$ (400 MHz, chloroform-d)

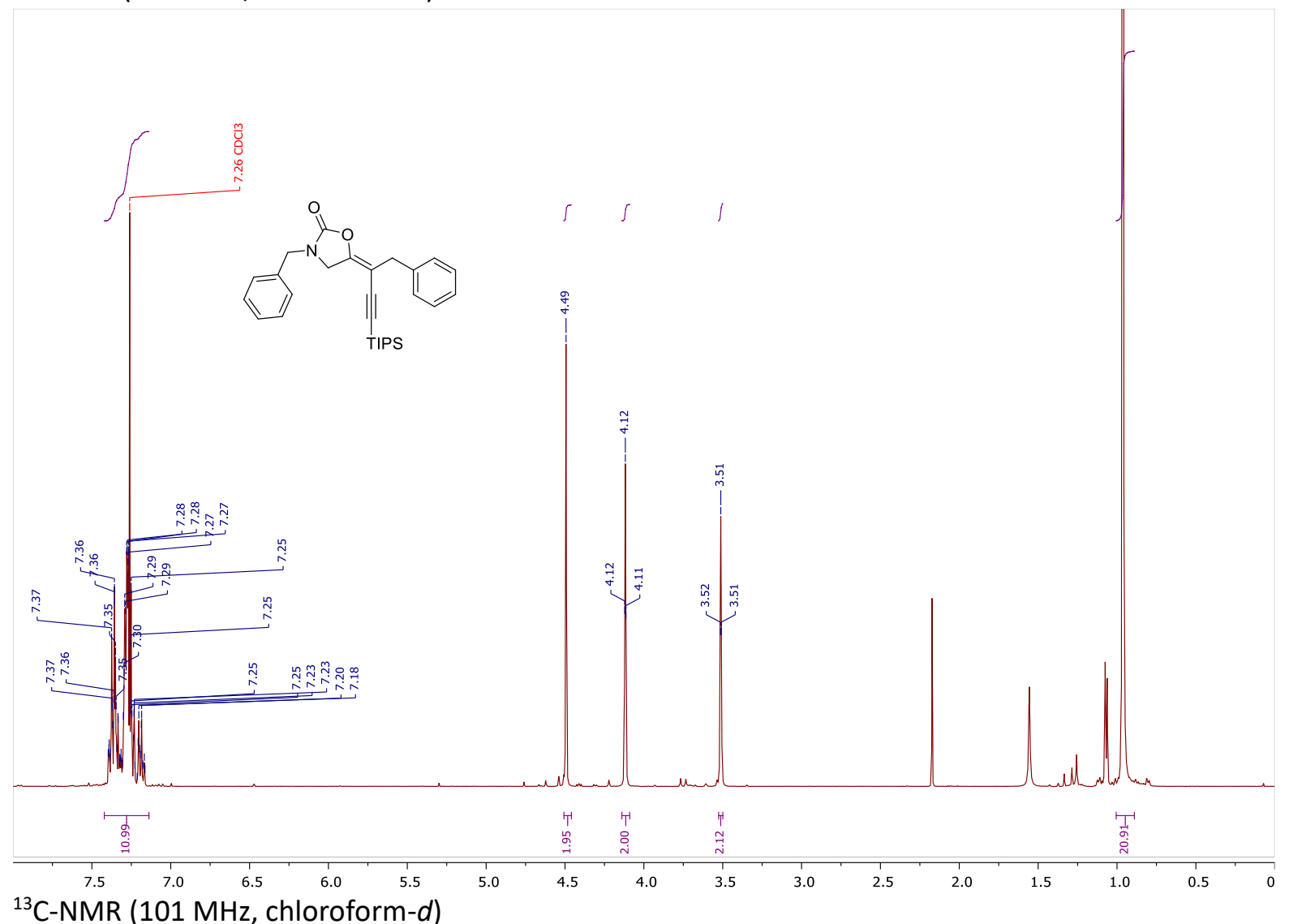

${ }^{13} \mathrm{C}-\mathrm{NMR}$ (101 MHz, chloroform-d)
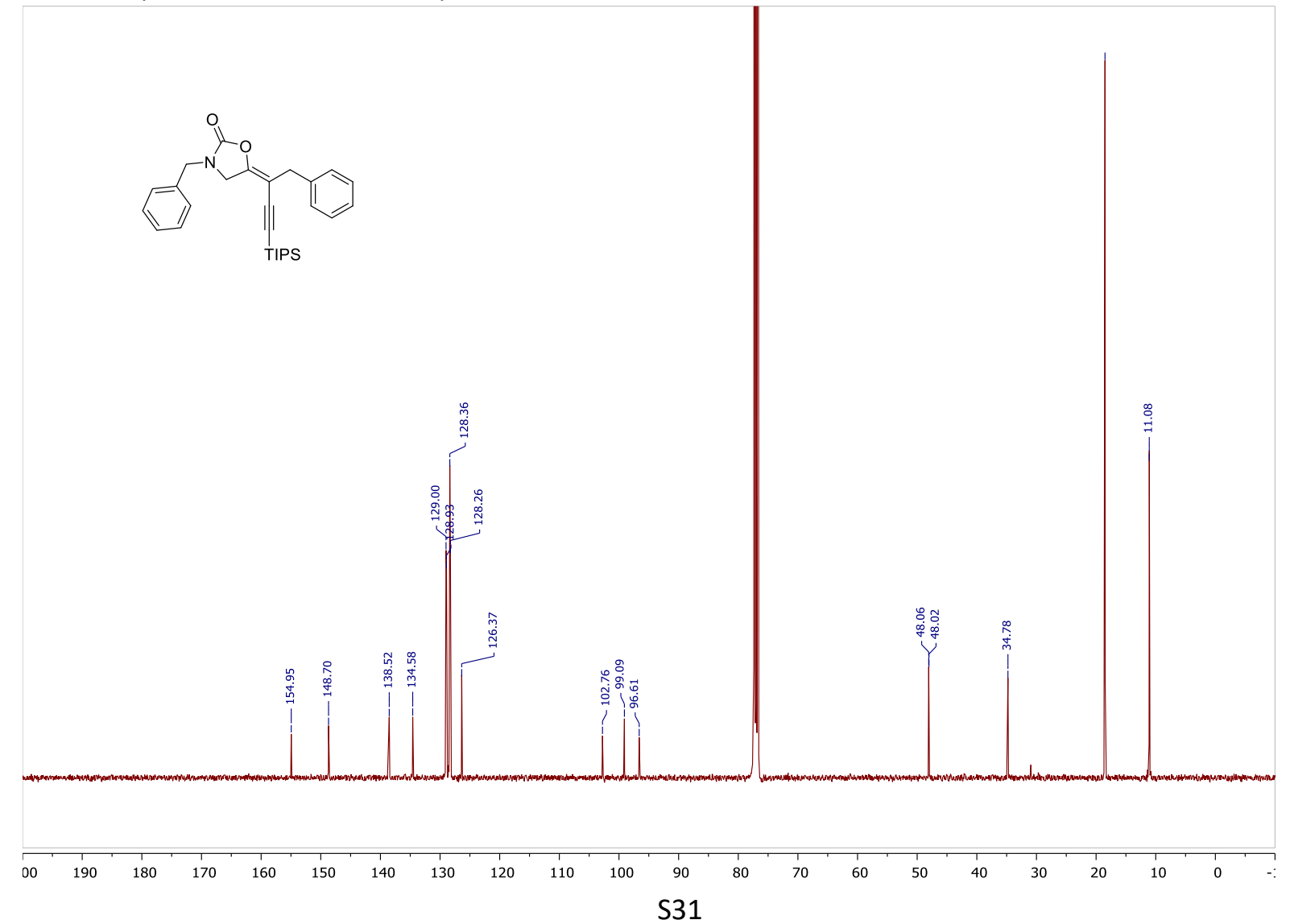
(E)-3-benzyl-5-(1-cyclopropyl-3-(triisopropylsilyl)prop-2-yn-1-ylidene)oxazolidin-2-one (5e)

${ }^{1} \mathrm{H}-\mathrm{NMR}(400 \mathrm{MHz}$, chloroform-d)

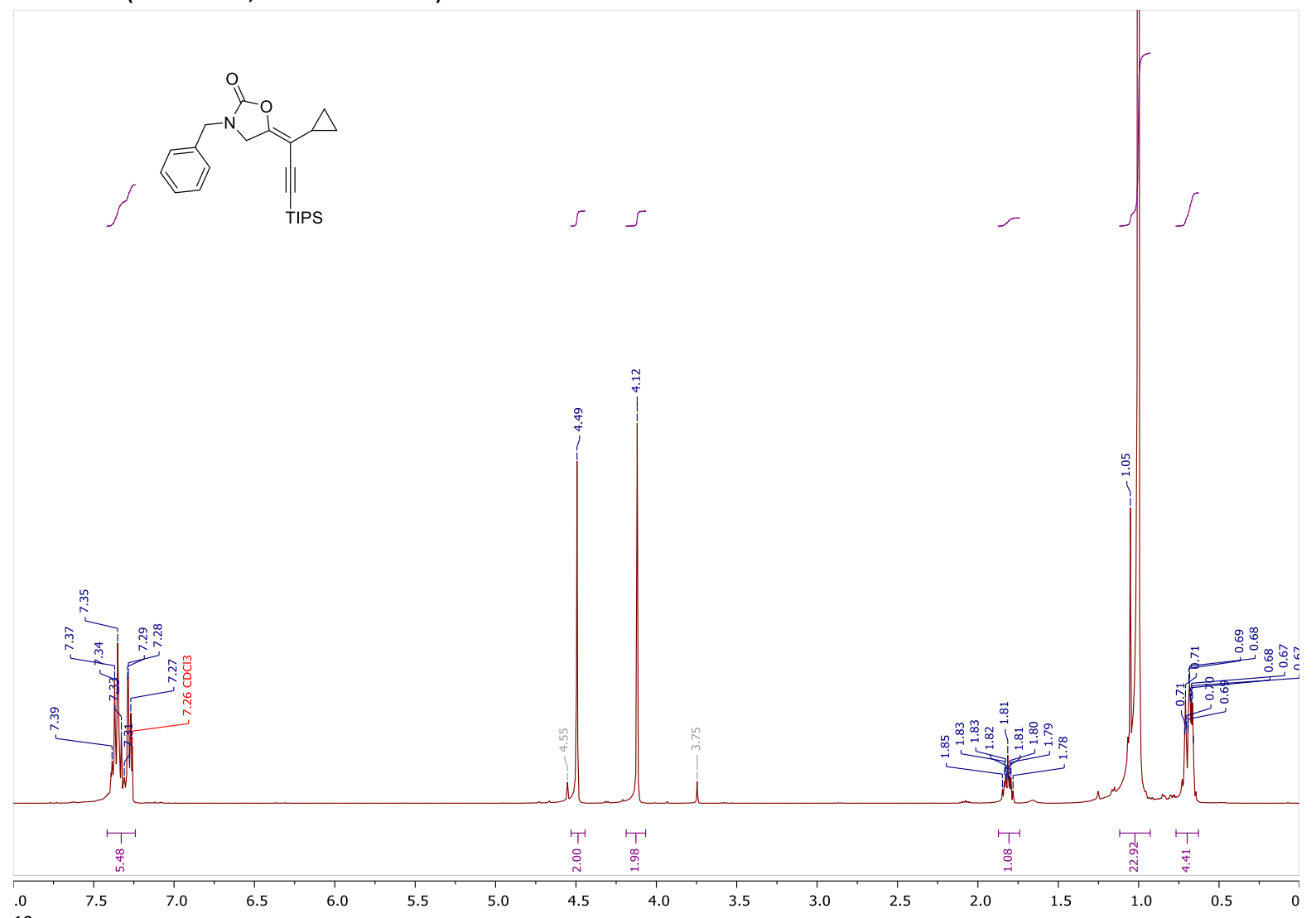

${ }^{13} \mathrm{C}-\mathrm{NMR}$ (101 MHz, chloroform-d)
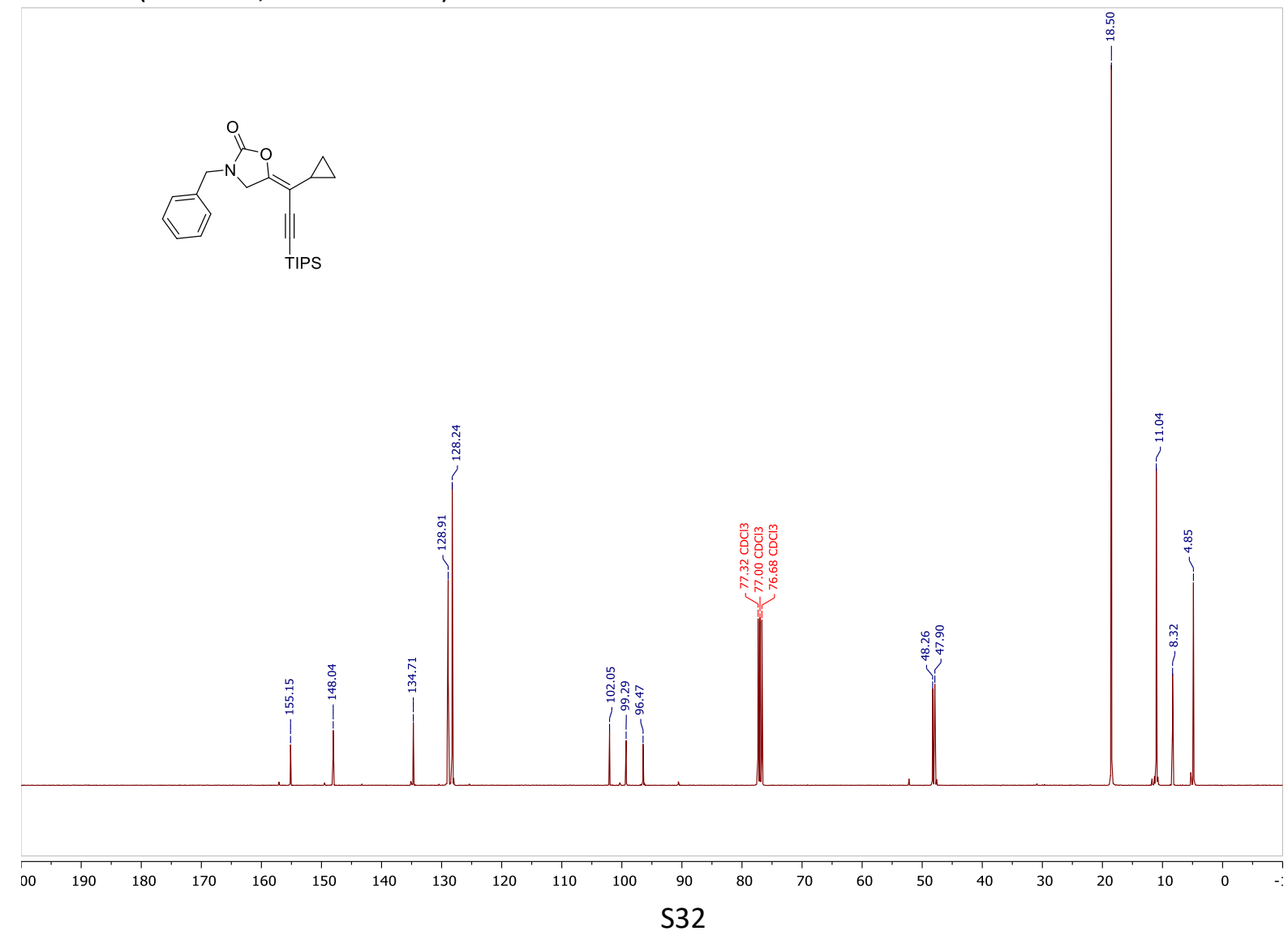
(E)-3-benzyl-5-(1-cyclopropyl-3-(triisopropylsilyl)prop-2-yn-1-ylidene)oxazolidin-2-one (5f)

${ }^{1} \mathrm{H}-\mathrm{NMR}$ (400 MHz, chloroform-d)

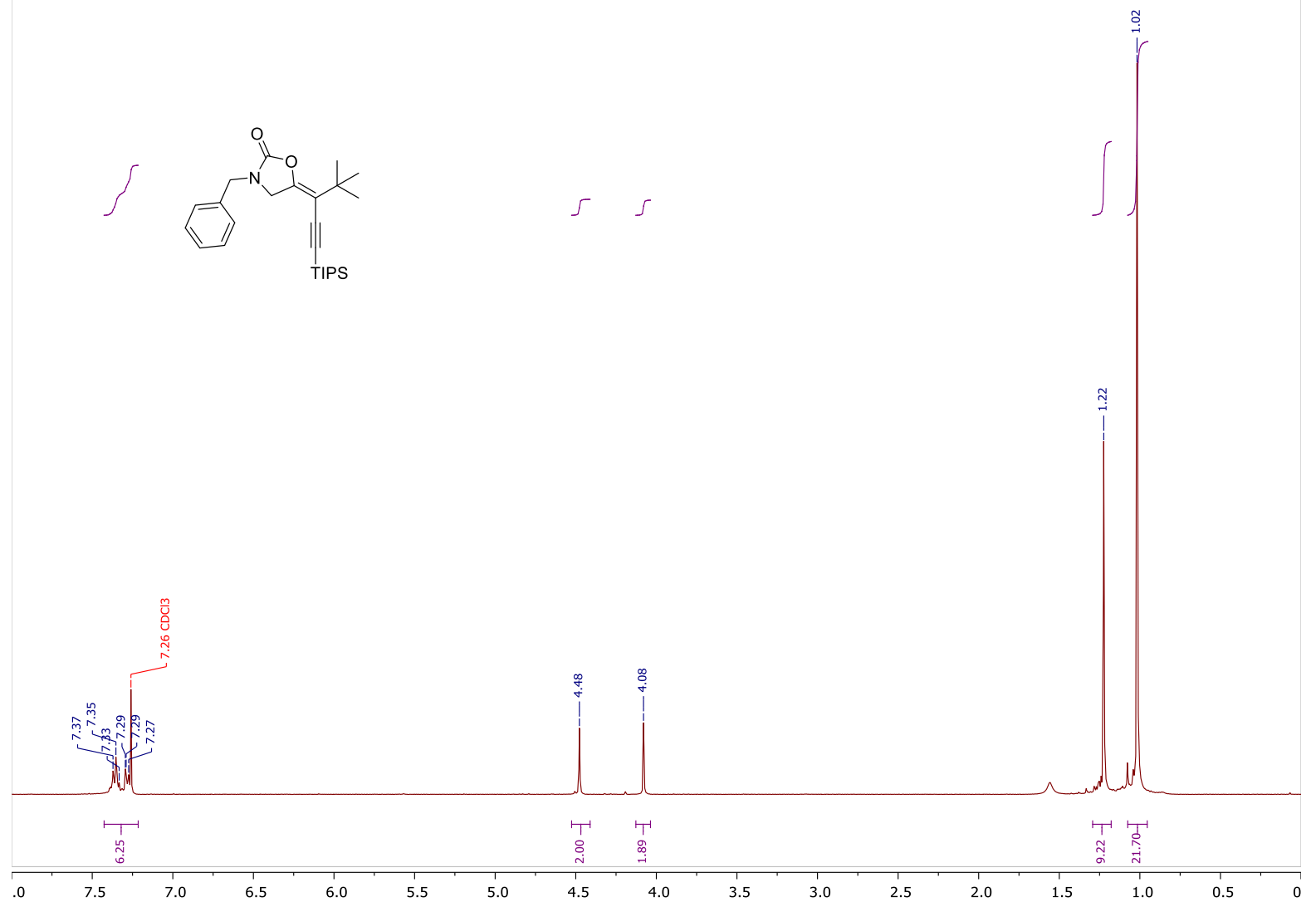

${ }^{13} \mathrm{C}-\mathrm{NMR}$ (101 MHz, chloroform-d)

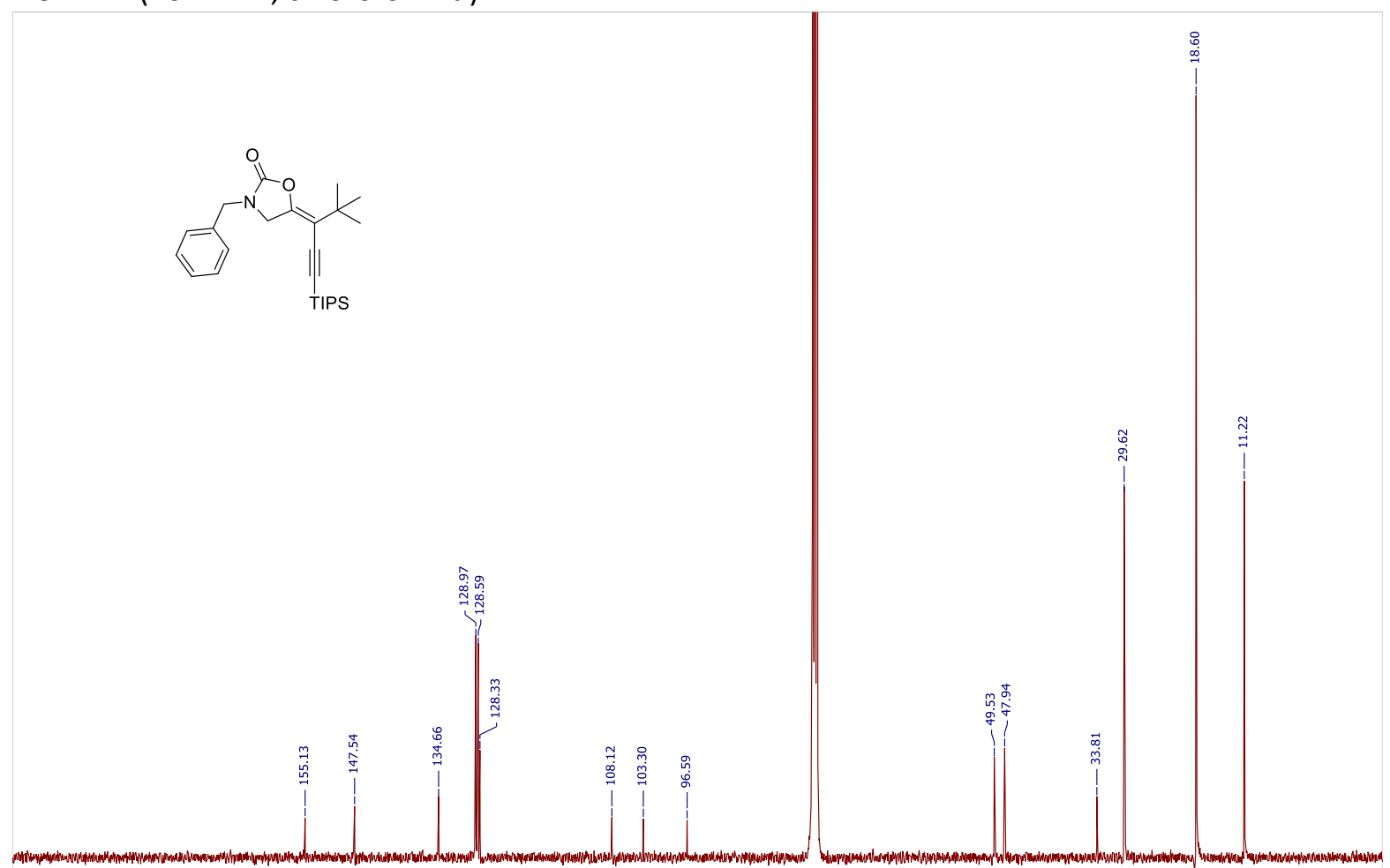

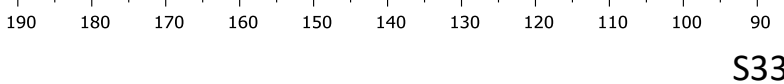


(E)-3-benzyl-5-(1-phenyl-3-(triisopropylsilyl)prop-2-yn-1-ylidene)oxazolidin-2-one (5g)

${ }^{1} \mathrm{H}-\mathrm{NMR}$ (400 MHz, chloroform-d)

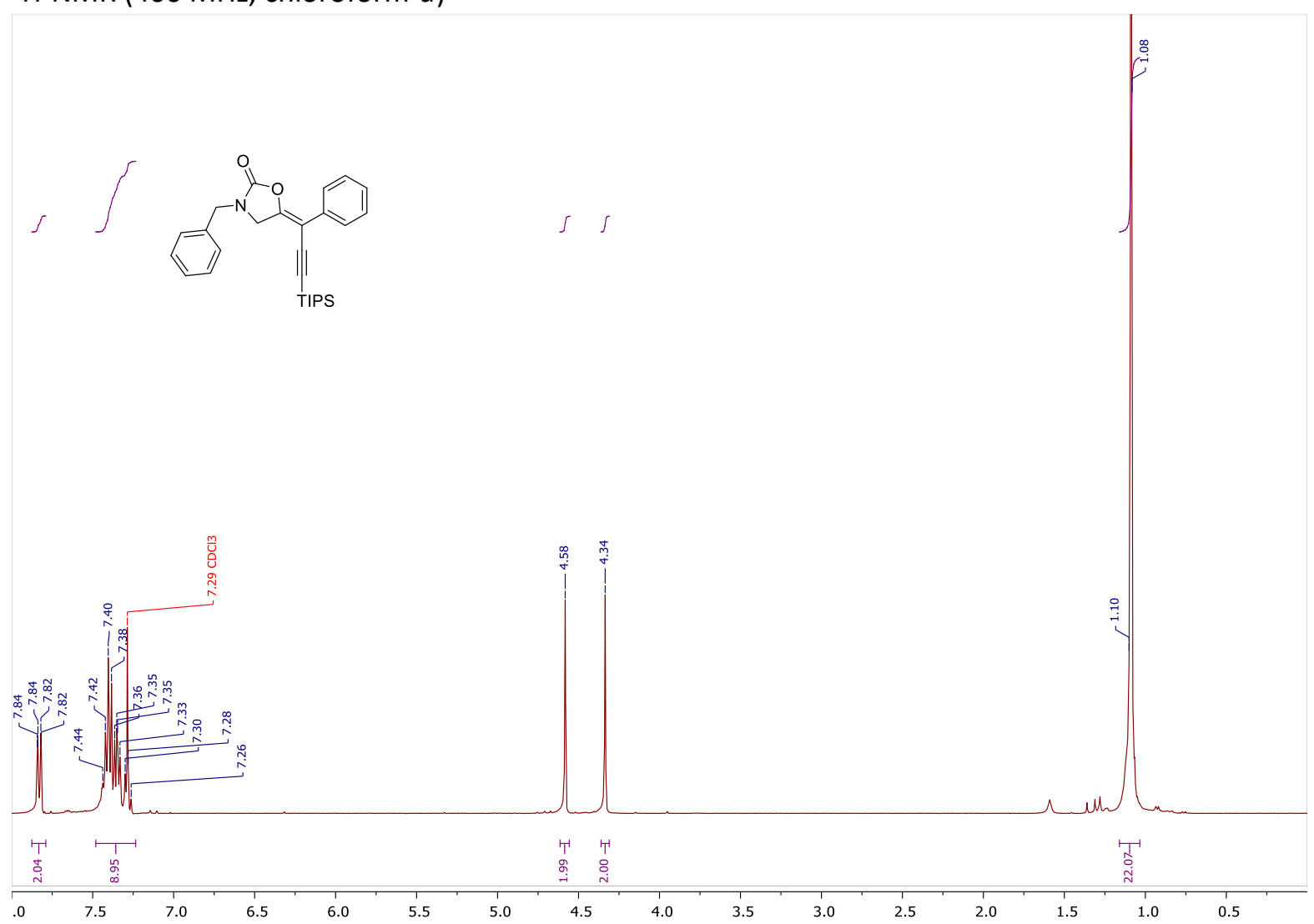

${ }^{13} \mathrm{C}-\mathrm{NMR}$ (101 MHz, chloroform-d)

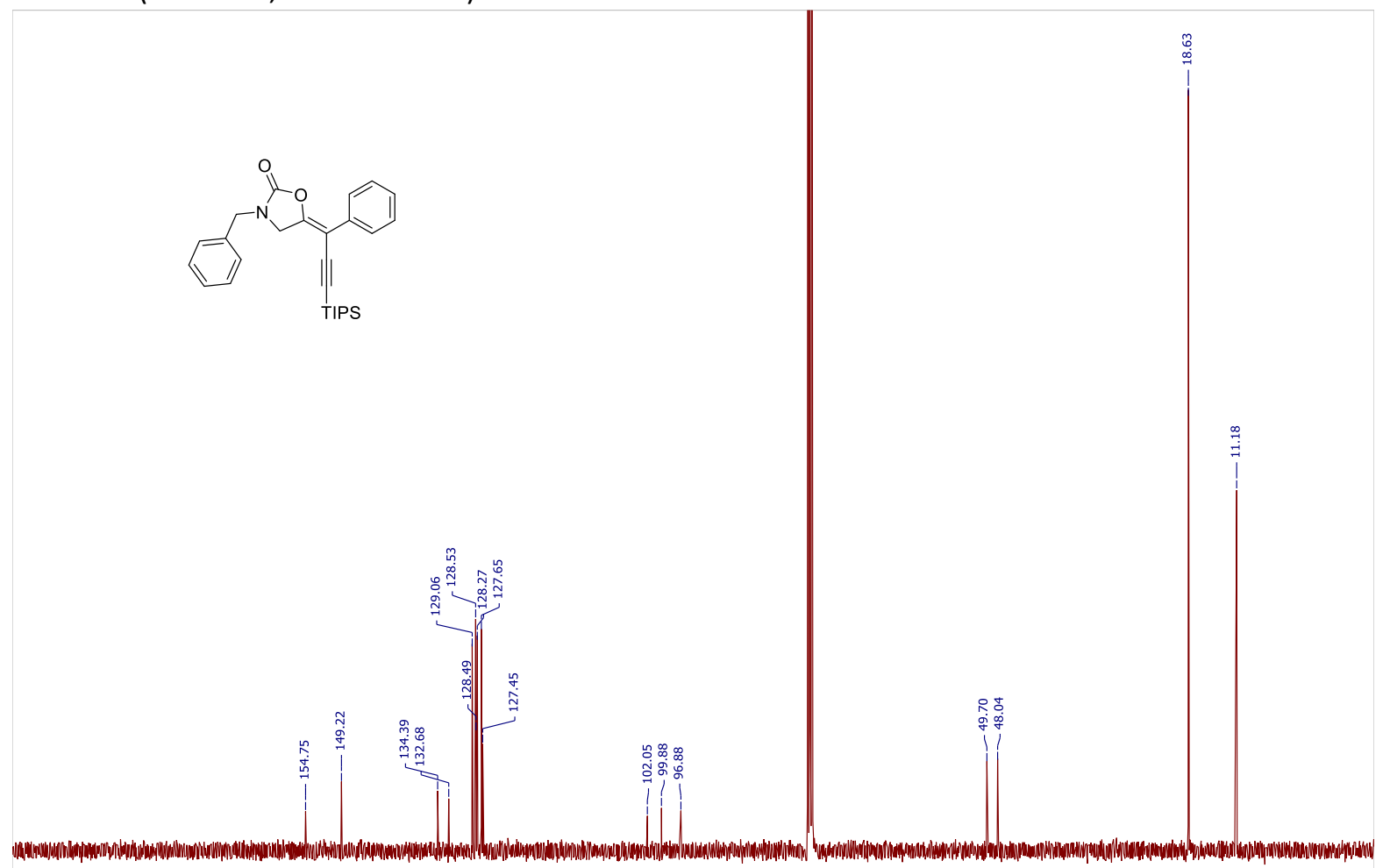

$190 \quad 180$

$170 \quad 160 \quad 150$

$140 \quad 130$

120 
(E)-3-methyl-5-(1-phenyl-3-(triisopropylsilyl)prop-2-yn-1-ylidene)oxazolidin-2-one (5h)

${ }^{1} \mathrm{H}-\mathrm{NMR}$ (400 MHz, chloroform-d)
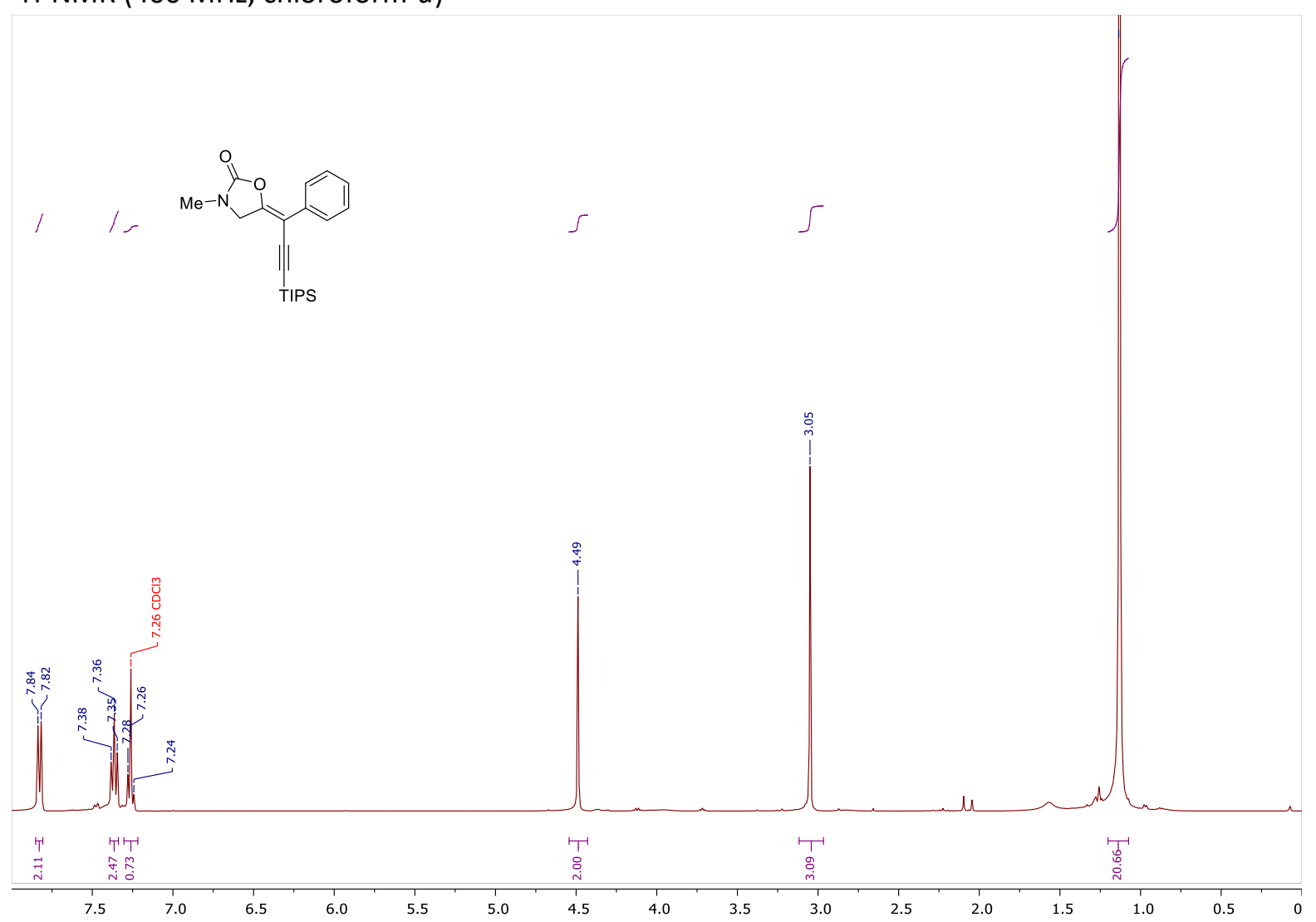

${ }^{13} \mathrm{C}-\mathrm{NMR}$ (101 MHz, chloroform-d)
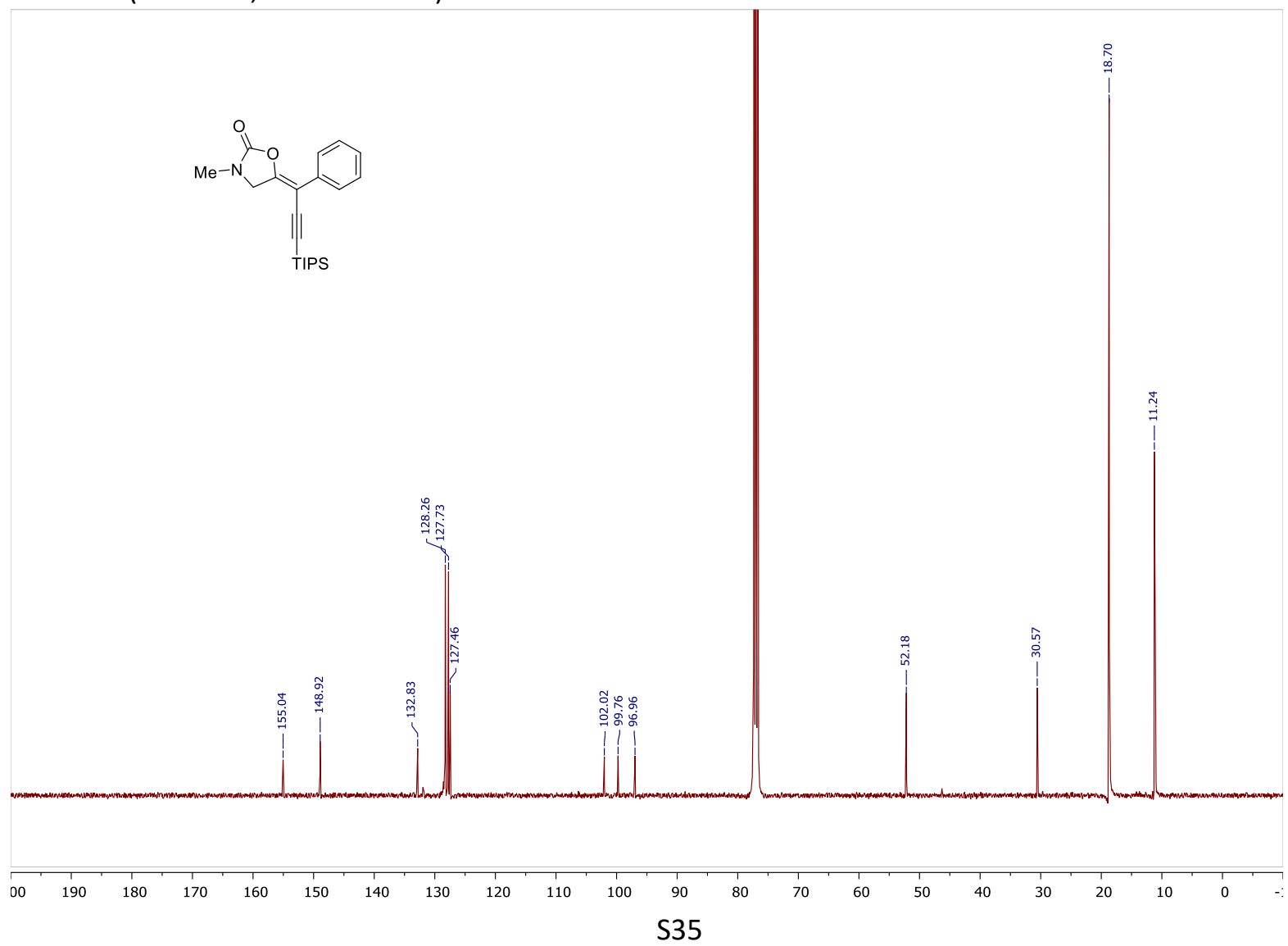
(E)-3-benzyl-5-(1-(4-methoxyphenyl)-3-(triisopropylsilyl)prop-2-yn-1-ylidene)oxazolidin-2-one (5i) ${ }^{1} \mathrm{H}-\mathrm{NMR}$ (400 MHz, chloroform-d)

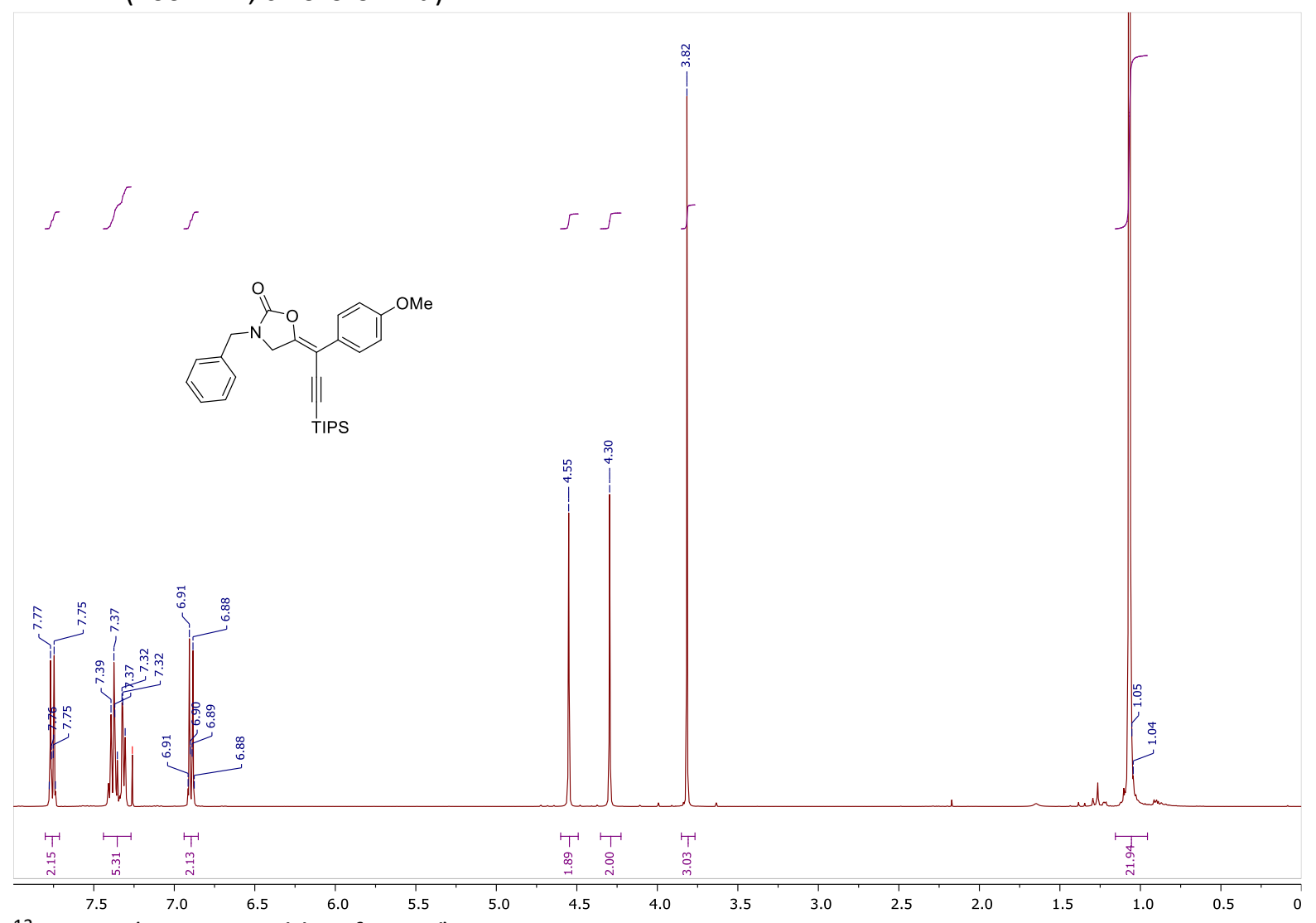

${ }^{13} \mathrm{C}-\mathrm{NMR}$ (101 MHz, chloroform-d)
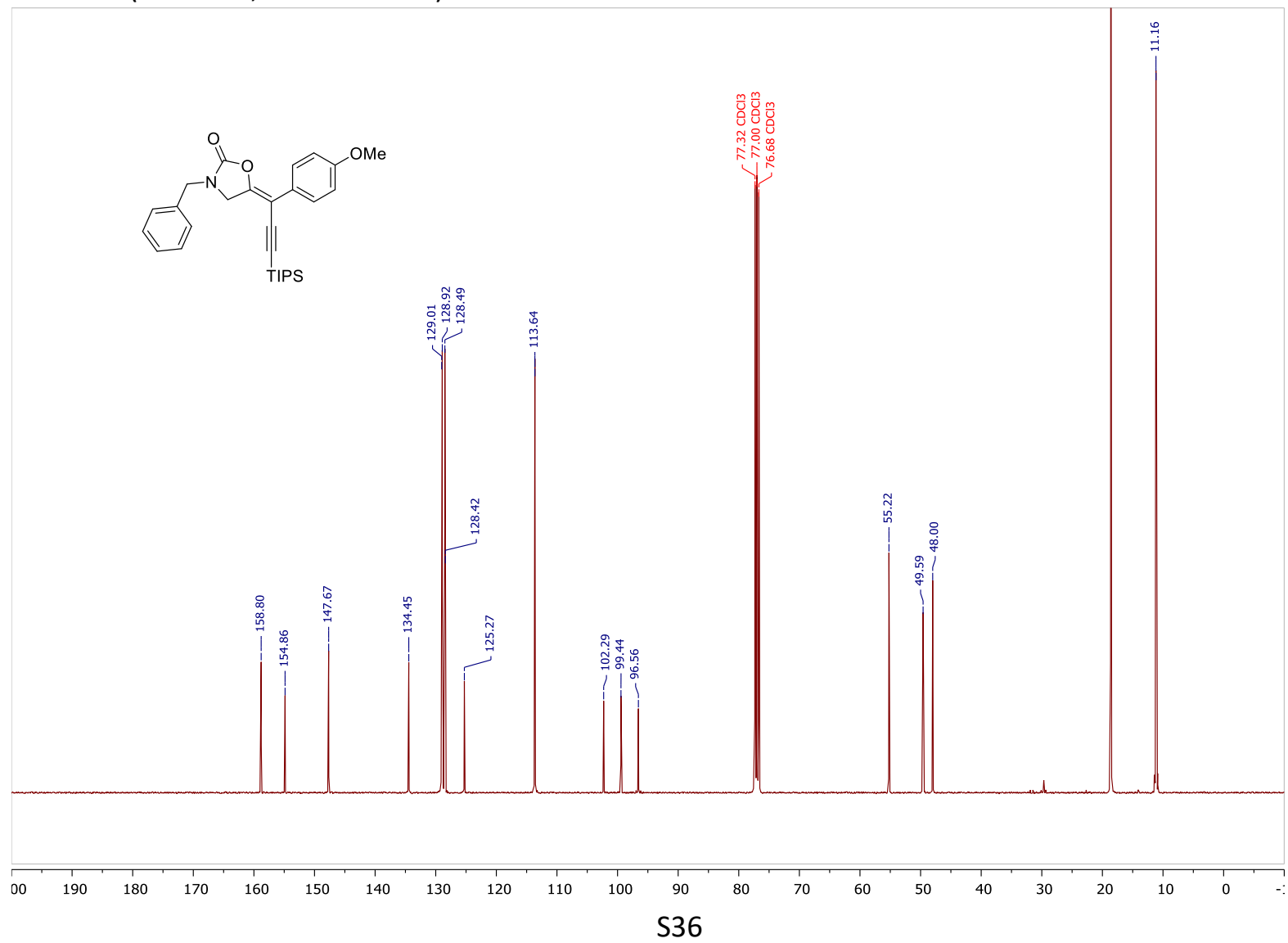
(E)-3-benzyl-5-(1-(4-(trifluoromethyl)phenyl)-3-(triisopropylsilyl)prop-2-yn-1-ylidene)oxazolidin-2-one

\section{(5i)}

${ }^{1} \mathrm{H}-\mathrm{NMR}$ (400 MHz, chloroform-d)
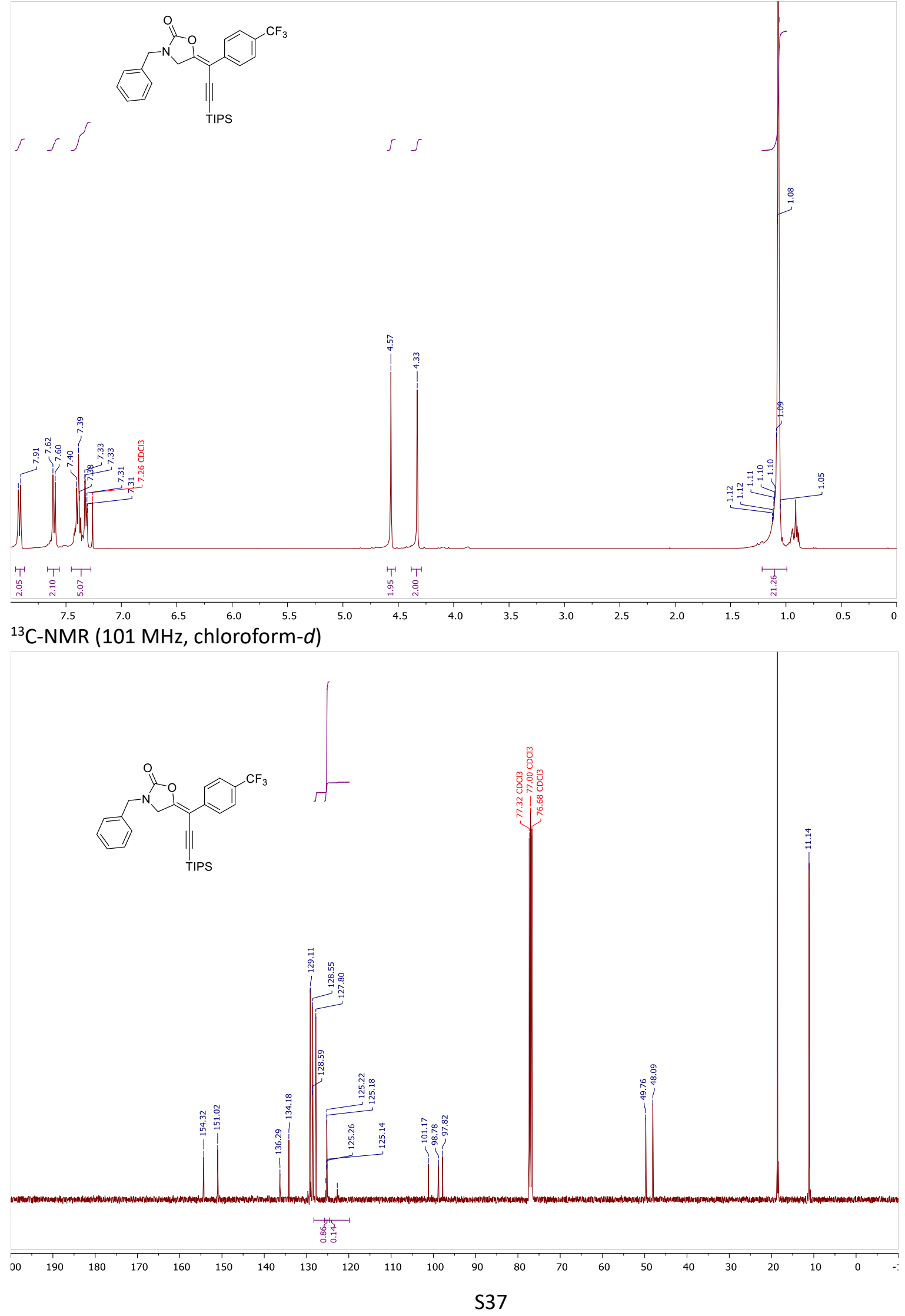
(E)-5-(1-(3-bromophenyl)-3-(triisopropylsilyl)prop-2-yn-1-ylidene)-3-phenyloxazolidin-2-one (5k) ${ }^{1} \mathrm{H}-\mathrm{NMR}(400 \mathrm{MHz}$, chloroform-d)

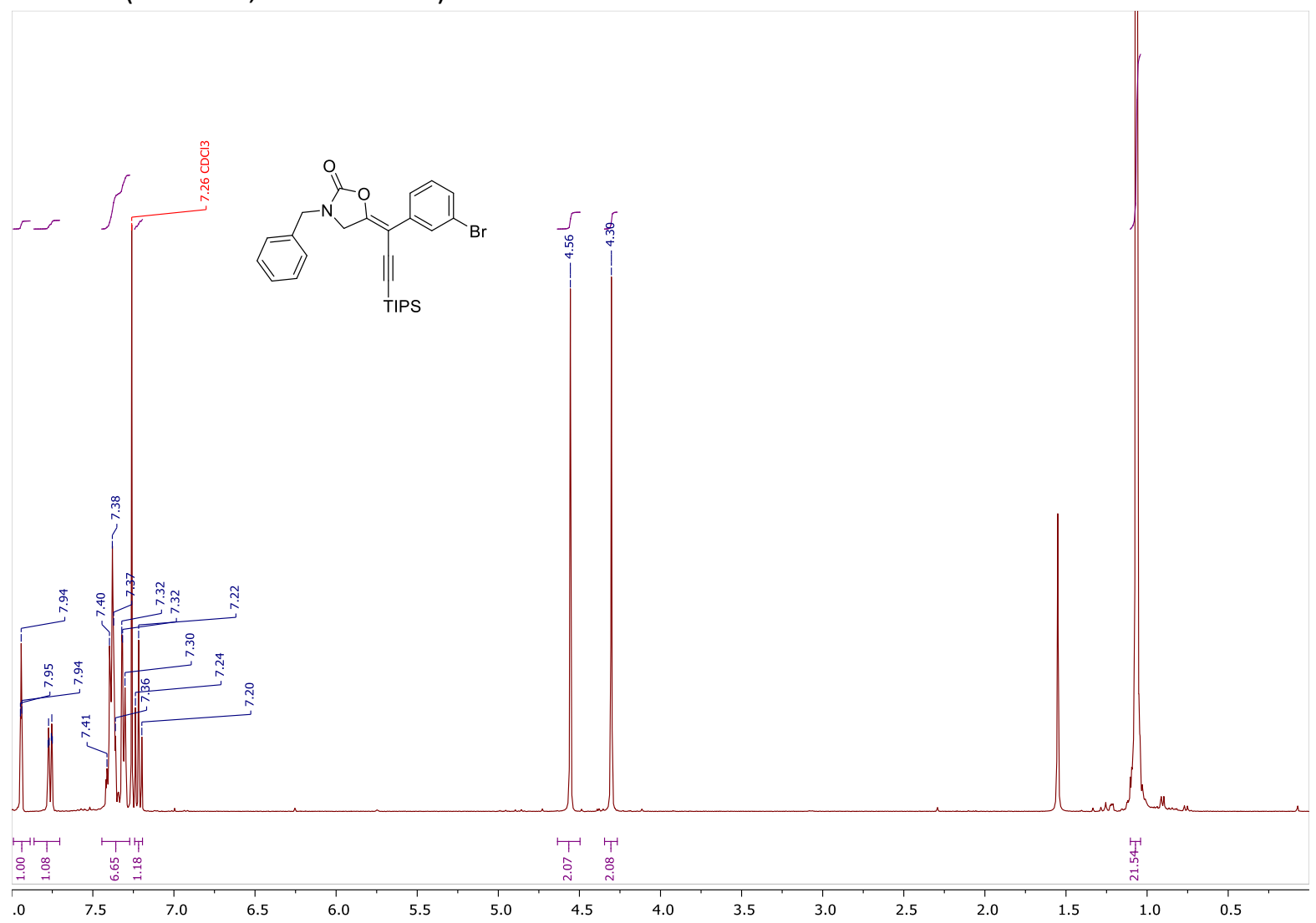
${ }^{13} \mathrm{C}-\mathrm{NMR}(101 \mathrm{MHz}$, chloroform-d)

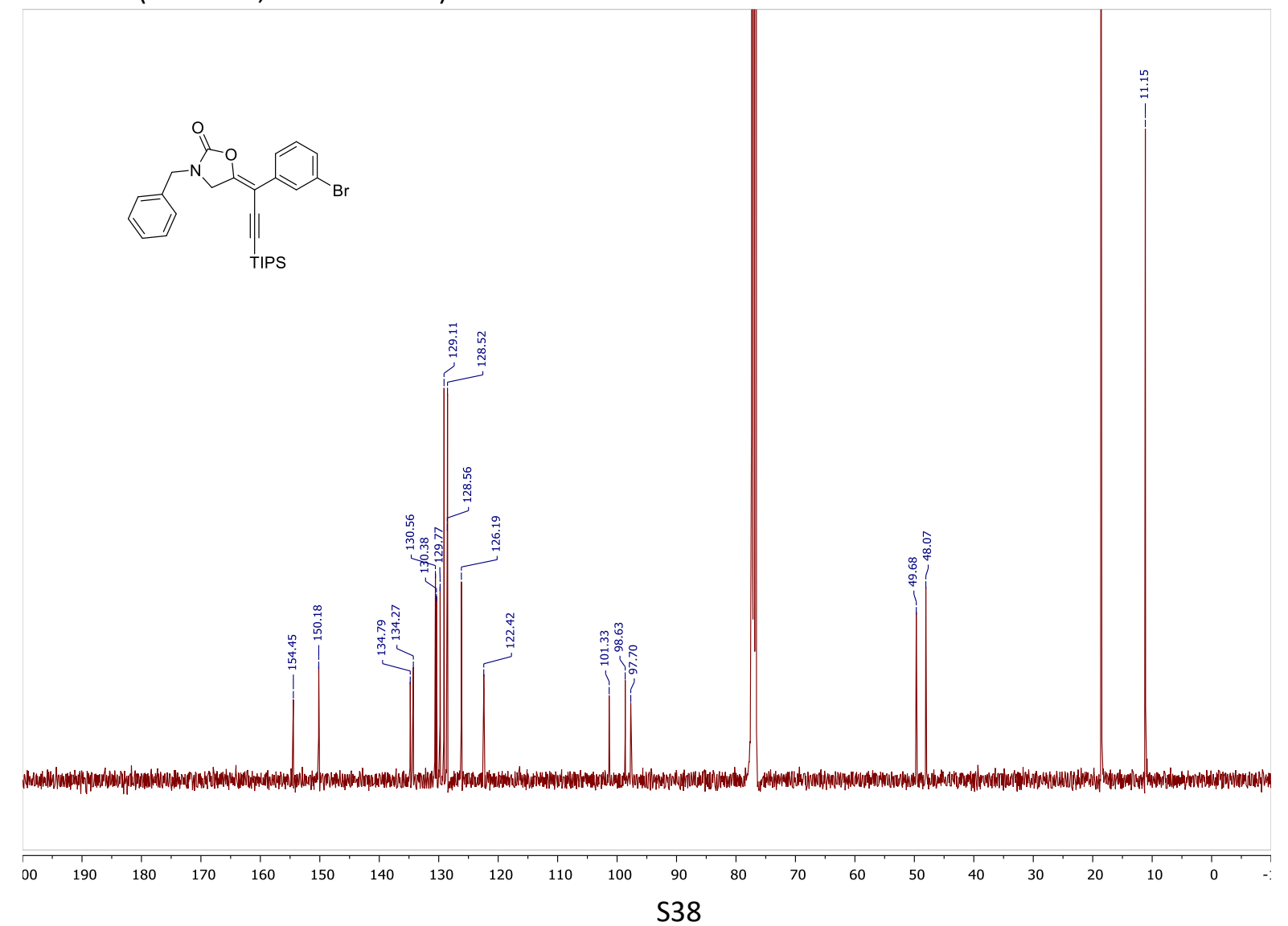


(E)-3-benzyl-5-(1-(3,5-dimethylphenyl)-3-(triisopropylsilyl)prop-2-yn-1-ylidene)oxazolidin-2-one (5I) ${ }^{1} \mathrm{H}-\mathrm{NMR}$ (400 MHz, chloroform-d)

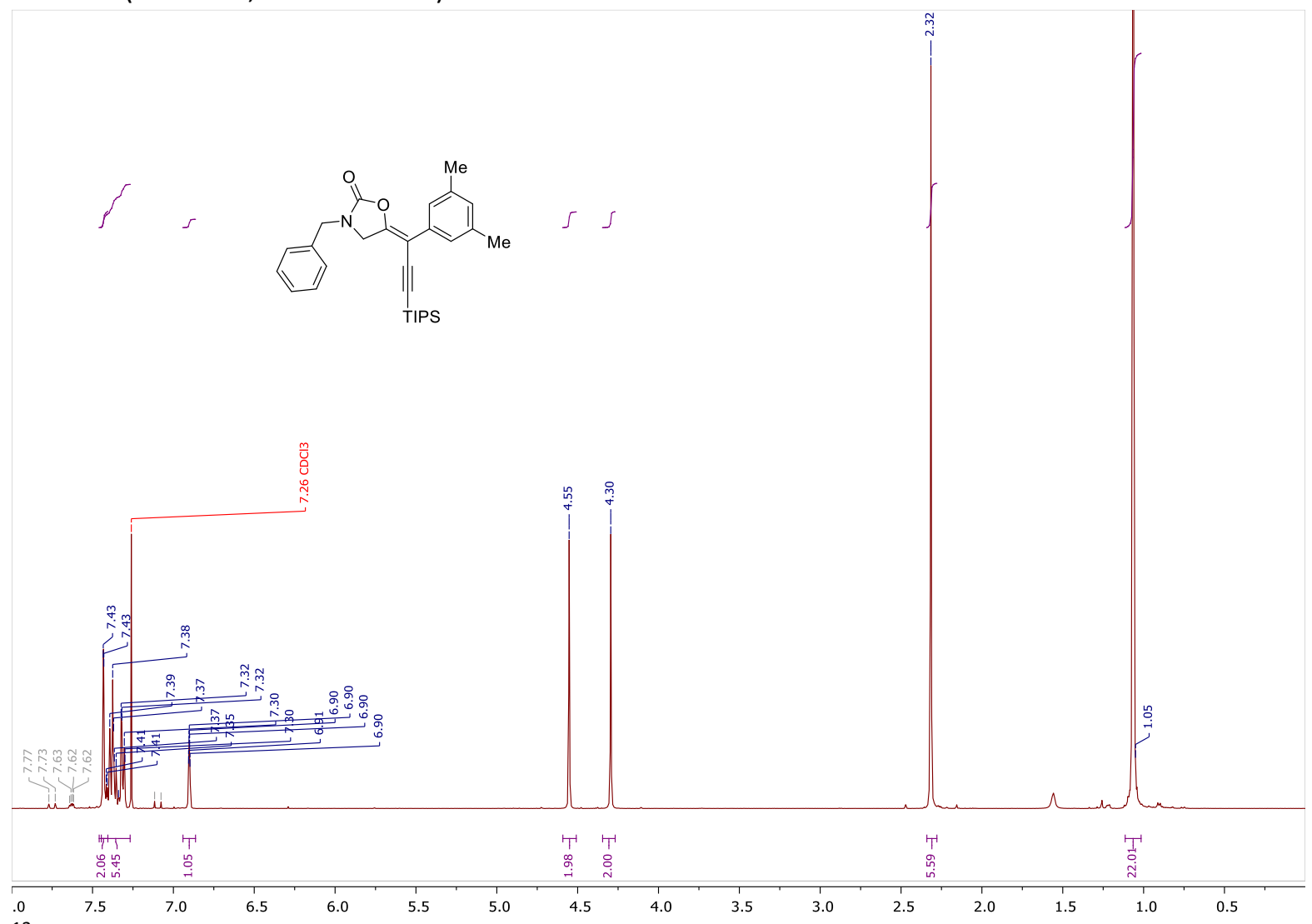
${ }^{13} \mathrm{C}-\mathrm{NMR}(101 \mathrm{MHz}$, chloroform-d)
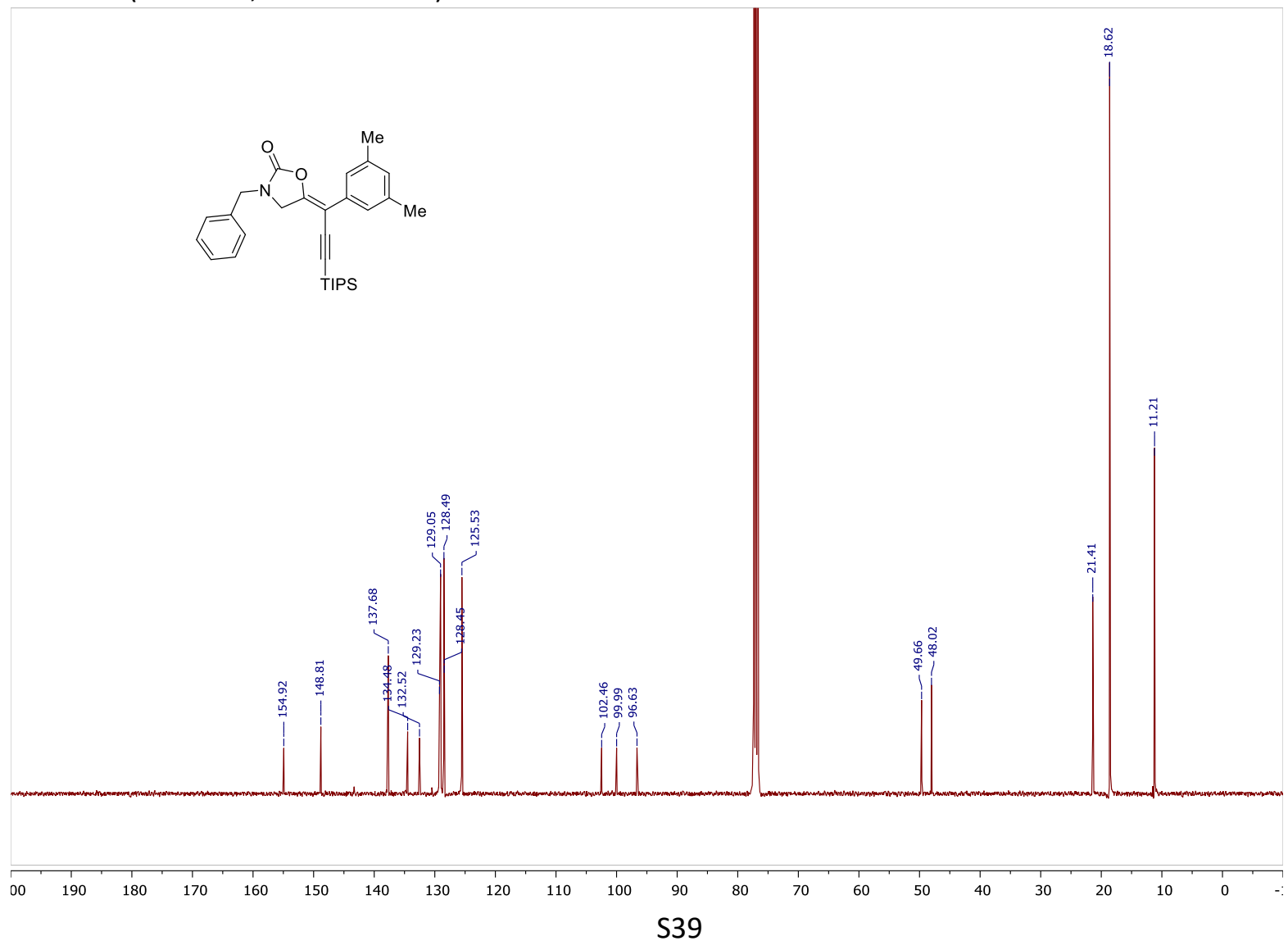
(Z)-3-benzyl-5-(1-(thiophen-2-yl)-3-(triisopropylsilyl)prop-2-yn-1-ylidene)oxazolidin-2-one (5m) ${ }^{1} \mathrm{H}-\mathrm{NMR}$ (400 MHz, chloroform-d)

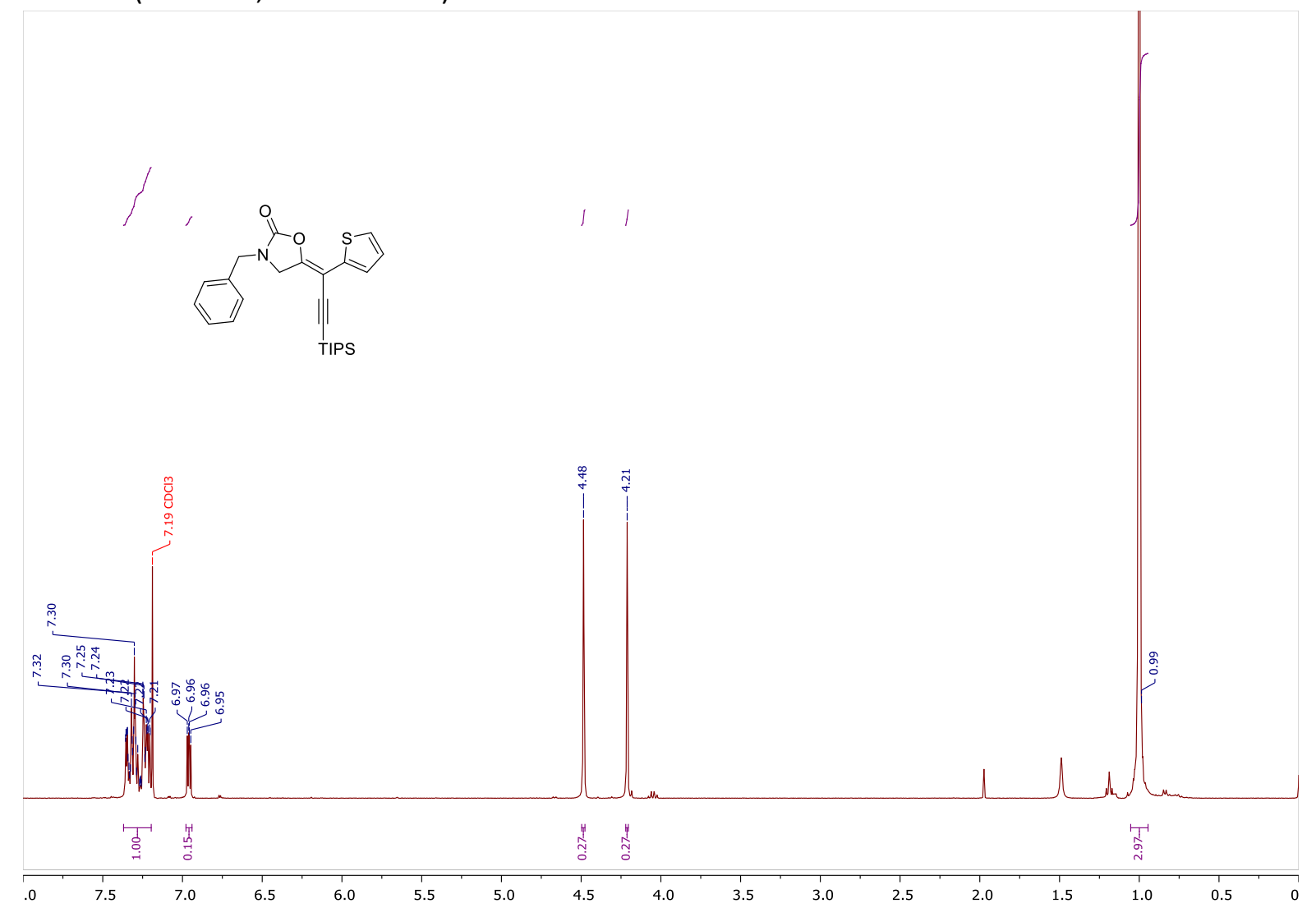

${ }^{13} \mathrm{C}-\mathrm{NMR}$ (101 MHz, chloroform-d)
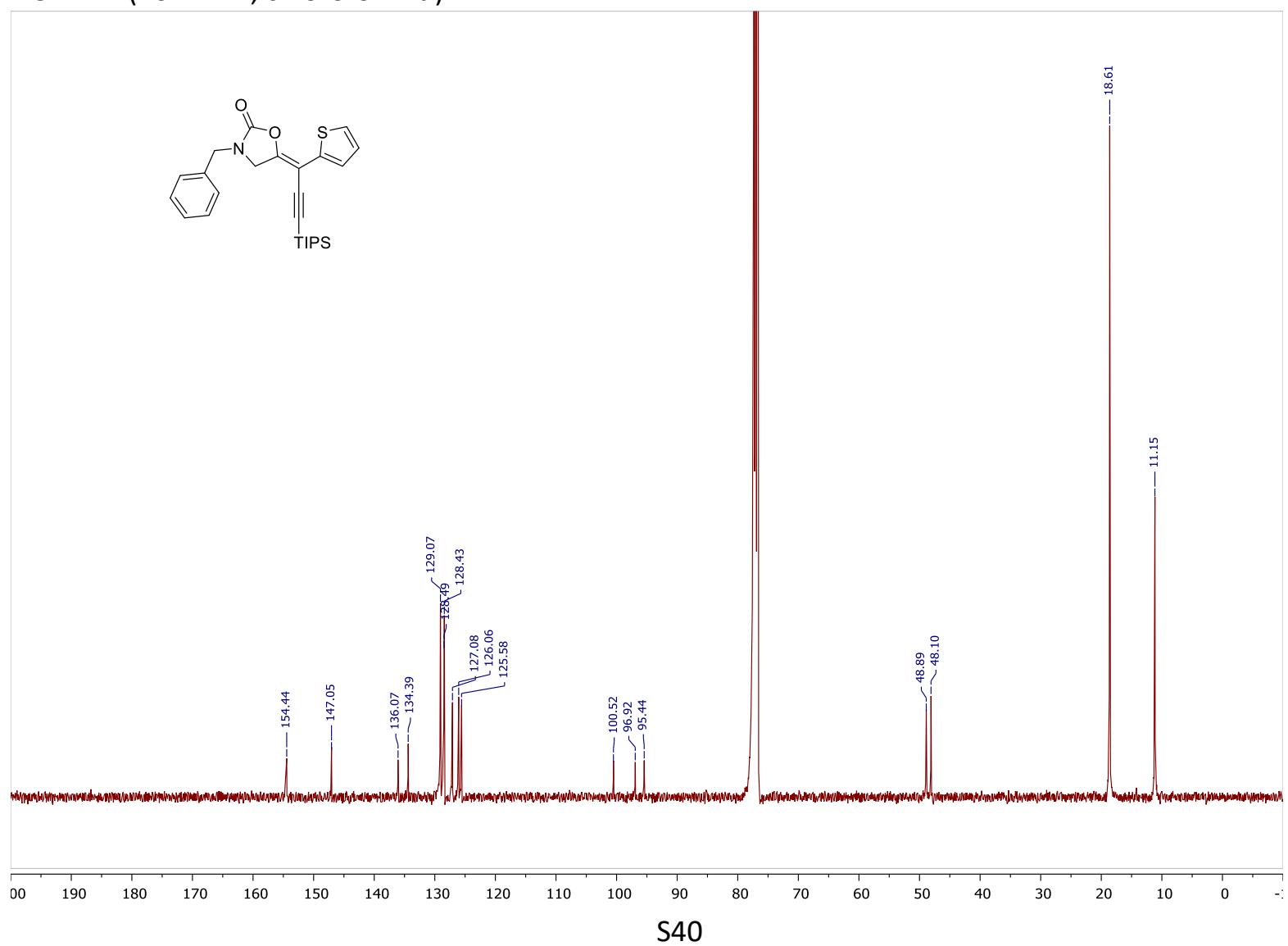
(E)-3-benzyl-5-(1-(p-tolyl)ethylidene)oxazolidin-2-one (9) and (Z)-3-benzyl-5-ethylideneoxazolidin-2-one (10) ${ }^{1} \mathrm{H}-\mathrm{NMR}$ (400 MHz, chloroform-d)

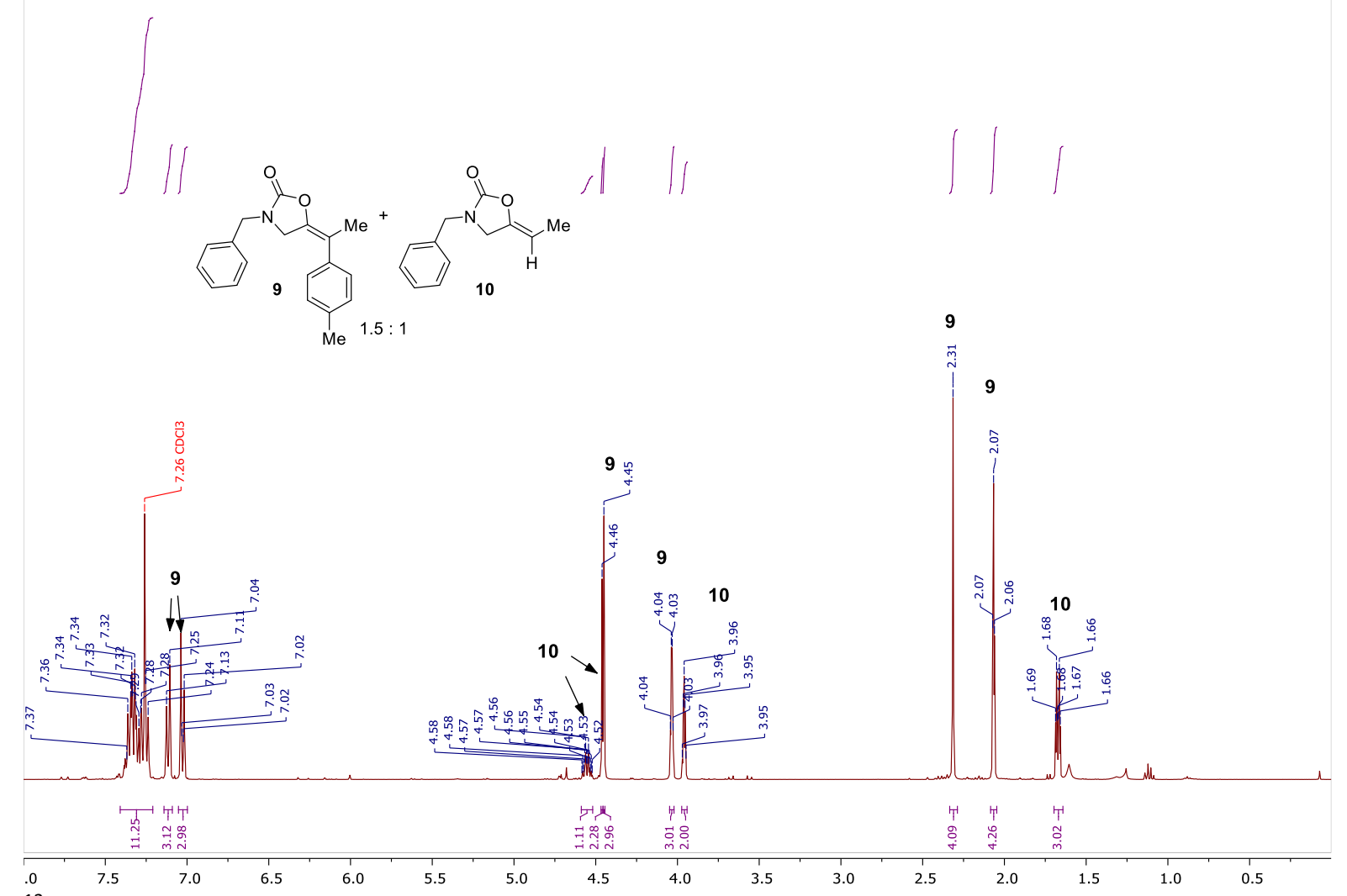
${ }^{13} \mathrm{C}-\mathrm{NMR}(101 \mathrm{MHz}$, chloroform-d)
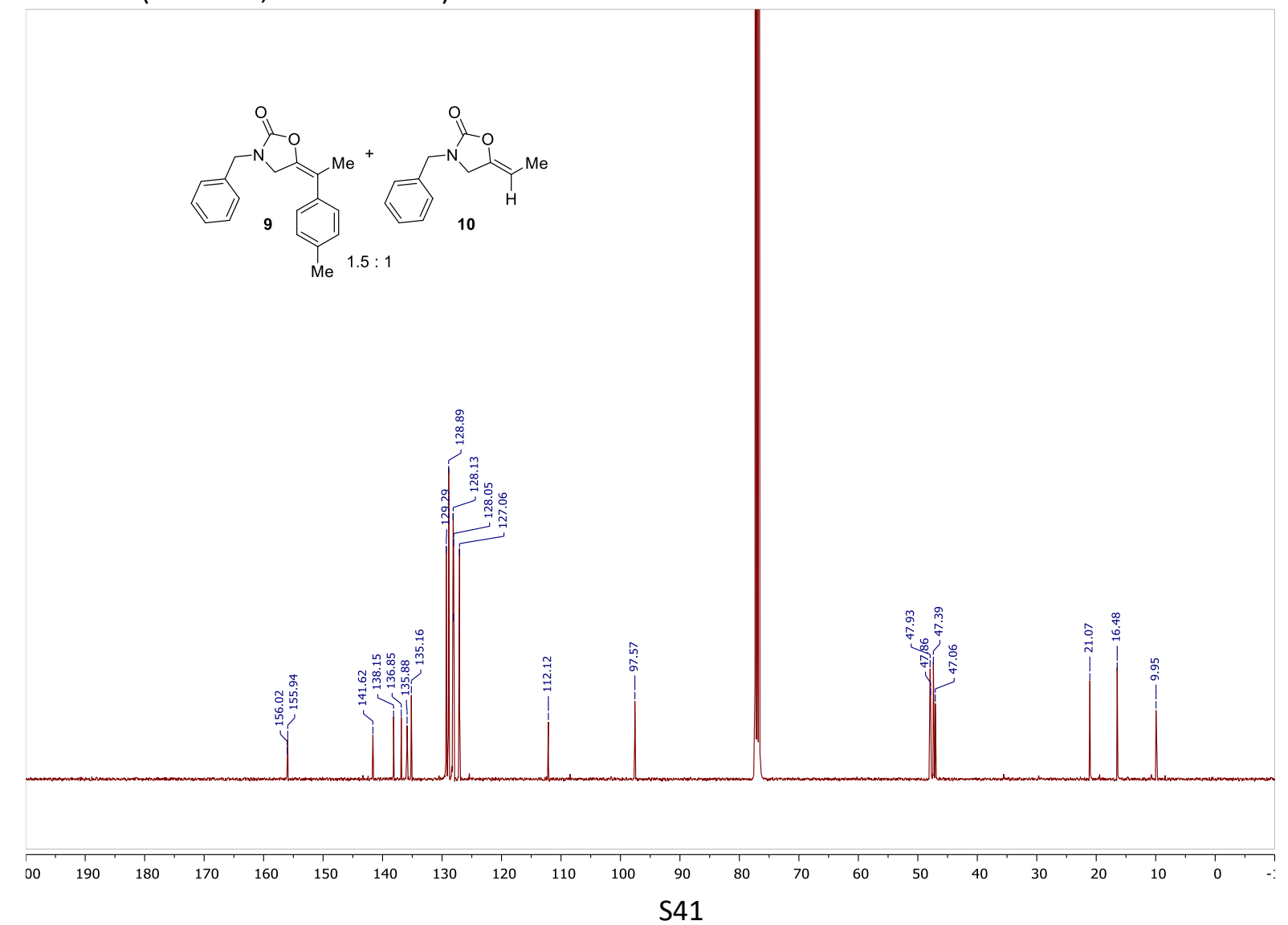